2. To: (Receiving Organization)

Plutonium Finishing PTant

5. Proj./Prog./Dept./Div.:

PFP

8. Originator Remarks:

Transmittal of Shielding Wall Calculations for PFP Vertical Calciner Glovebox

11. Receiver Remarks
3. from: (originating organization)

Criticality and Shielding

6. Design Authority/ Design Agent/Cog. Engr.:

W. D. Wittekind
11A. Design Basel ine Document? [] res [X] No

12. Major Assm. Dwg. No.:

$N / A$

13. Permit/Permit Application No.: $N / A$

14. Required Response Date:

$N / A$

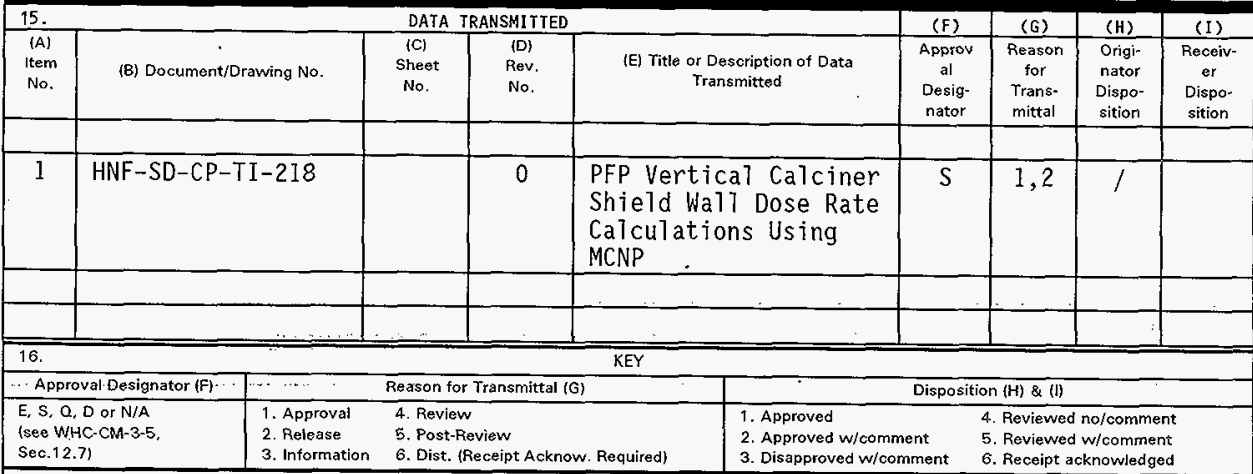

17. SIGNATURE/DISTRIBUTION

(See Approval Designator for required signatures)

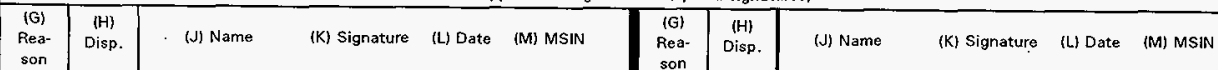

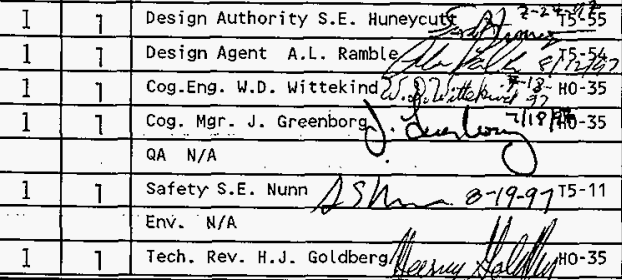

\begin{tabular}{|l|l}
\hline 18. & 19
\end{tabular}

Warrewi. Wittebued

Signature of EDT

Originator

8D-7400-172-2 (05/96) GEF097
19.

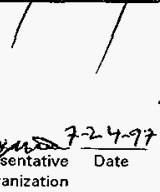

20.

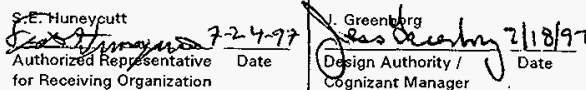

21. DOE APPROVAL (if required) Ctrl. No.

[] Approved

[] Approved w/comments

[.] Di sapproved w/comments 
HNF-SD-CP-TI-218, Rev, 0

\section{PFP Vertical Calciner Shield Wall Dose Rate Calculations Using MCNP}

Warren D. Wittekind

Fluor Daniel Northwest, Richland, WA 99352

U.S. Department of Energy Contract DE-AC06-96RL13200

EDT/ECN: 620293 UC: 507

Org Code: $403 \quad$ Charge Code: E56571

B\&R Code: EW7003000 Total Pages: 86

Key Words: Calciner, Vertical Calciner, Dose Rate, Exposure, ALARA

Abstract: This report yields a neutron shield wall design for a full time occupancy dose rate of $0.25 \mathrm{mrem} / \mathrm{h}$. ORIGEN2 generated gamma ray spectrum and neutron intensity for plutonium. MCNP modeled the calciner glovebox and room for reflection of neutrons off concrete walls and ceiling. Neutron calculations used MCNP in mode $n, p$ to include neutron capture gammas. Photon calculations used MCNP in mode $p$ for gamma rays.

Neutron shield with lower $137.16 \mathrm{~cm}$ (4.5 feet) of $12.7 \mathrm{~cm}$ (5 inch) thick Lucite ${ }^{\circledR}$ land $0.3175 \mathrm{~cm}(0.125$ inch) stainless steel on both sides, and upper $76.2 \mathrm{~cm}$ (2.5 feet) of $10.16 \mathrm{~cm}$ (4 inch) thick Lucite ${ }^{\circledR}$ and $1.905 \mathrm{~cm}$ (0.75 inch) thick glass on each side gave a total weighted dose rate of $0.23 \mathrm{mrem} / \mathrm{h}$, fulfilling the design goa 1 . Lucite ${ }^{\circledR}$ is considered to be equivalent to Plexiglas ${ }^{\circledR}$ since both are methylmethacrylate polymers.

${ }^{1}$ Lucite is a registered trademark of $E$. I. du Pont de Nemours and co. 2plexiglas is a registered trademark of Rohm \& Haas.

TRADEMARK DISCLAIMER. Reference herein to any specific comercial product, process, or service by trade name, trademark, manufacturer, or otherwise, does not necessarily constitute or imply its endorsement, recommendation, or favoring by the United States Government or any agency thereof or its contractors or subcontractors.

Printed in the United States of America. To obtain copies of this document, contact: WHC/BCS Document Control Services, P.0. Box 1970, Mailstop H6-08, Richland WA 99352, Phone (509) 372-2420; Fax (509) 376-4989.

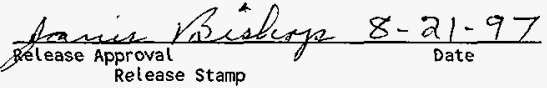
Release Stamp

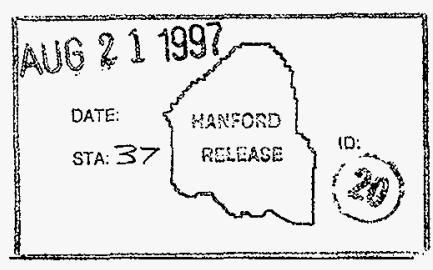


HNF-SD-CP-TI-218 Rev. 0

\section{PFP VERTICAL CALCINER SHIELD WALL DOSE RATE CALCULATIONS USING MCNP}


HNF-SD-CP-TI-218 Rev. 0

CONTENTS

1.0 INTRODUCTION AND SUMMARY . . . . . . . . . . . . . . . . . . . 1

2.0 DESCRIPTION OF SYSTEM AND FACILITY . . . . . . . . . . . . . 2

3.0 PROCESS FLOW DESCRIPTION . . . . . . . . . . . . . . . . . . . . . 2

4.0 GLOVEBOX MODELING FOR SHIELDING CALCULATIONS

4.1 CONCENTRATION AND COMPOSITION OF THE FISSILE MATERIAL . . . . 5

4.1 .1 Feed Solution . . . . . . . . . . 5

4.1.2 Vertical Calciner Charge . . . . . . . . . . . . . 6

4.1 .3 Calcine $\left(\mathrm{PuO}_{2}\right)$ Product . . . . . . . . . . . . 6

4.2 ROOM 230C MODELING ASSUMPTIONS . . . . . . . . . . . . . . 6

4.3 GLOVEBOX . . . . . . . . . . . . . . 6

4.3.1 Calciner Section of Glovebox . . . . . . . . . . . 7

4.3.2 Waste Section of Glovebox ........... . . 7

4.3.3 Connecting Section of Glovebox . . . . . . . . . . . 8

4.4 FEED SYSTEM . . . . . . . . . . . . . . . . . . . . . . 8

4.4.1 Feed Tanks . . . . . . . . . . . . . 8

4.4.2 Other Feed System Volumes . . . . . . . . . . . 9

4.5 VERTICAL CALCINER . . . . . . . . . . . . . . . . . 9

4.5.1 The Lower Section . . . . . . . . . . . . . 9

4.5.2 The Upper Section ........... . . . . . . 10

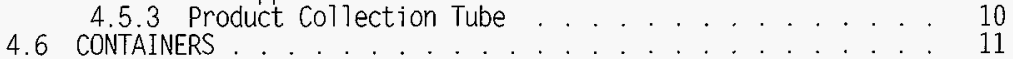

4.7 SCRUBBER SYSTEM

5.0 EVALUATION AND RESULTS . . . . . . . . . . . . . 11

5.1 PLUTONIUM INVENTORY OF VERTICAL CALCINER GLOVEBOX . . . . . 11

5.2 PLUTONIUM ACTIVITY CALCULATION BY ORIGEN2 $\ldots \ldots \ldots$. . . . . . . . 12

5.3 COMPONENT DOSE RATE CALCILATION BY MCNP $\ldots \ldots$

5.4 EFFECTIVE DOSE RATE CALCULATION BY WEIGHTING

5.5 COMPARISON OF LUCITE AND LEXAN NEUTRON SHIELDING MATERIAL : 14

5.6 COMPENSATION FOR INCREASED $(\alpha, n)$ IN PLUTONIUM NITRATE
SOLUTION

5.7 DOSE RATE PATHWAYYS TO OPERATOR BEHIND NEUTRON SHIELLD . . . . 16

5.8 DOSE RATE ADJACENT TO CALCINER GLOVEBOX SOURCES $\ldots \ldots$

5.9 HANDS AROUND CONTAINERS . . . . . . . . . . . . . . . . . . 18

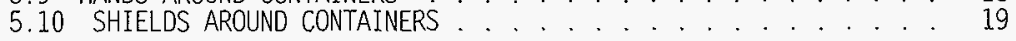

6.0 REFERENCES . . . . . . . . . . . . . . . . . . 21 
HNF-SD-CP-TI-218 Rev. 0

APPENDICES

APPENDIX A

INDEPENDENT REVIEW COMMENTS AND CHECKLIST . . . . . . . . . . . . . . A-1

APPENDIX B

ORIGEN2 PLUTONIUM INPUT FILE AND OUTPUT RESULTS . . . . . . . . . . . . . . B-1

APPENDIX C

NEUTRON SHIELDED OPERATOR DOSE RATE MCNP INPUT FILES . . . . . . . . c-1

APPENDIX D

HAND DOSE RATE MCNP INPUT FILES . . . . . . . . . . . . . . . . D-1

APPENDIX E

MISCELLANEOUS REFERENCE PAGES . . . . . . . . . . . . . . . . . . E-1

\section{LIST OF FIGURES}

Figure 1. Vertical Calciner Glovebox and Neutron Shield Wall. . . . . . 3

Figure 2. Vertical Calciner Glovebox Interior Measurements. . . . . . . 4

\section{LIST OF TABLES}

Table 4.1 Average Inventory Isotopic Composition . . . . . . . . . . . 5

Table 4.2 Off-Gas Filter Dimensions . . . . . . . . . . . . . . . . . . . . 10

Table 5.1 Lucite ${ }^{\circledR}$ Neutron Shield . . . . . . . . . . . . . . . . . . . 13

Table 5.2 Lexan® Neutron Shield . . . . . . . . . . . . . . . . . . . . . . 14

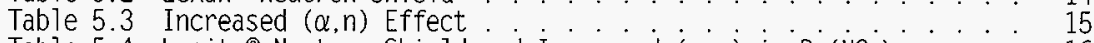

Table 5.4 Lucite ${ }^{\circledR}$ Neutron Shield and Increased $(\alpha, n)$ in $\mathrm{Pu}_{\left(\mathrm{NO}_{3}\right)_{4}} \ldots . .16$

Table 5.5 Radiation Dose Rate From The Different Pathways . . . . . . 17

Table 5.6 Radiation Dose Adjacent to Unshielded Glovebox . . . . . . . . 18

Table 5.7 Hand Dose Rate From Receipt Cans . . . . . . . . . . . . . . . 19

Table 5.8 Storage Can Cylindrical Shields - Dose Rates . . . . . . . . . 20

Table 5.9 Storage Can Square Shields - Dose Rates . . . . . . . . 20 
HNF-SD-CP-TI-218 Rev. 0

Title: $\quad$ PFP Vertical Calciner Shield Wall Dose Rate Calculations using MCNP

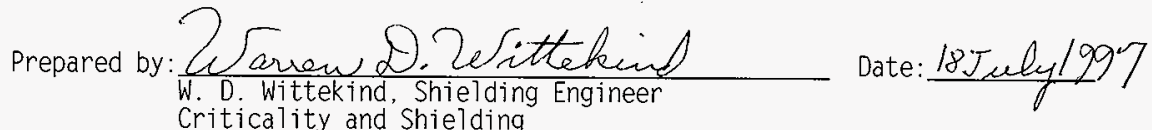

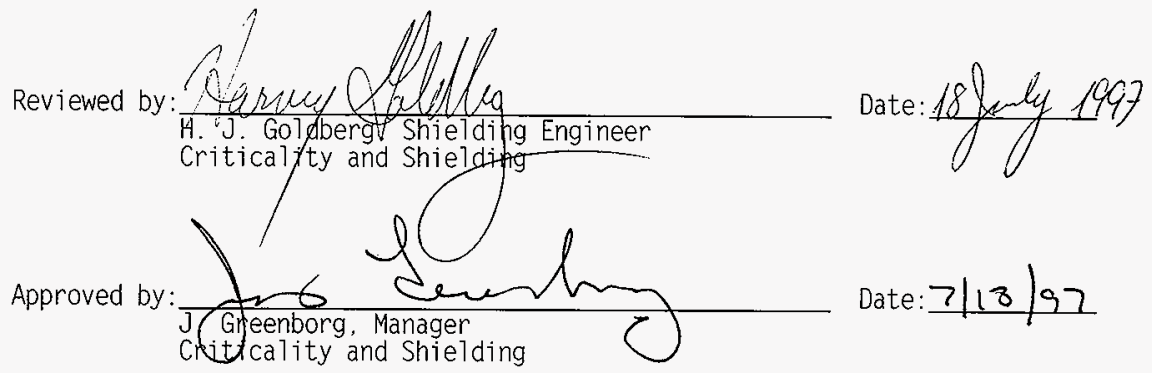


HNF-SD-CP-TI-218 Rev. 0

This page intentionally left blank 
HNF-SD-CP-TI-218 Rev. 0

PFP VERTICAL CALCINER SHIELD WALL
DOSE RATE CALCULATIONS USING MCNP

\subsection{INTRODUCTION AND SUMMARY}

This evaluation supports the fulfillment of the $0.25 \mathrm{mrem} / \mathrm{h}$ full time occupancy dose rate goal due to the calcining process inside this glovebox.

Assumptions for the source term included $350 \mathrm{~g} \mathrm{Pu} / \mathrm{L}$ in the $\mathrm{Pu}\left(\mathrm{NO}_{3}\right)_{4}$ solution with 10 Liters in both the feed tank (receipt) and feed tank (pump). The source term assumed in the vertical calciner is $3.5 \mathrm{~kg} \mathrm{Pu}$. The source term assumed $4.5 \mathrm{~g} \mathrm{Pu} / \mathrm{cm}^{3}$ in the $\mathrm{PuO}_{2}$ compound in the two $1 \mathrm{~L}$ receipt cans and in four $0.5 \mathrm{~L}$ slip lid cans (tota] $4.0 \mathrm{~L}$ in cans). This quantities yielded a total of $7.0 \mathrm{~kg}$ Pu in the feed tanks, $3.5 \mathrm{~kg} \mathrm{Pu}$ in the calciner, and $18.0 \mathrm{~kg}$ $\mathrm{Pu}$ in cans as the source term for the calciner glovebox. The dose rates from the different ${ }^{240} \mathrm{Pu}$ wt\% plutonium inventories were weighted according to their quantities for an effective average dose rate.

The design goal of $0.25 \mathrm{mrem} / \mathrm{h}$ for ful 1 time occupancy can be accomplished using $10.16 \mathrm{~cm}$ to $12.70 \mathrm{~cm}$ (4 in. to 5 in.) of Lucite ${ }^{\oplus 1}$ plastic neutron shielding with Stainless Steel and glass on each side of the Lucite per the present design. The present design uses $12.70 \mathrm{~cm}$ (5 in.) of Lucite with $0.3175 \mathrm{~cm}(0.125 \mathrm{in}$.) stainless steel on both sides on the lower 137.16 $\mathrm{cm}(4.5 \mathrm{ft})$ and uses $10.16 \mathrm{~cm}$ (4 in.) of Lucite with $1.905 \mathrm{~cm}(0.75 \mathrm{in.})$ glass on both sides on the upper $76.20 \mathrm{~cm}(2.5 \cdot \mathrm{ft})$.

Lucite is equivalent to Plexiglas ${ }^{\oplus 2}$ since both are methylmethacrylate (: $\mathrm{CH}_{2}-\mathrm{CH}_{3}-\mathrm{C}:-\mathrm{CO}_{2}-\mathrm{CH}_{3}$ or $\mathrm{C}_{5} \mathrm{H}_{8} \mathrm{O}_{2}$ ) polymers. The main difference it that these are the trade names used by different manufacturers. The processing may be different, leading to different length polymer chains, but similar elemental composition and similar densities are anticipated. In this document, when Lucite $^{\oplus}$ is stated, this is to be considered equivalent to Plexiglas ${ }^{\oplus}$ as far as neutron and gamma (photon) shielding is concerned.

Operator dose rate was summed for the gamma, capture gamma, and neutron dose contributions from the feed tanks, the calciner, and the plutonium oxide collected in cans. Annual dose rates were scaled proportionately for the three categories of ${ }^{240} \mathrm{Pu}$ material. The conversion from flux to dose used the ANS 6.1.1 1991 standard with the factor of 2 multiplied times the calculated neutron dose, as specified in ANS 6.1.1 1991.

The Lucite shield achieved the design goal of $0.25 \mathrm{mrem} / \mathrm{h}$, replacement with Lexan ${ }^{\theta_{3}}$ calculated to $0.30 \mathrm{mrem} / \mathrm{h}$ exceeding the design goal using the same plutonium source and shield thickness as for the Lucite calculation. The Lexan ${ }^{\otimes}$ is polycarbonate $\left(: 0-\mathrm{C}_{6} \mathrm{H}_{4}-\mathrm{C}\left(\mathrm{CH}_{3}\right)_{2}\left(\mathrm{C}_{6} \mathrm{H}_{4}\right) \mathrm{OCO}\right.$ : or $\left.\mathrm{C}_{6} \mathrm{H}_{34} \mathrm{O}_{3}\right)$ and was modeled as $1.2 \mathrm{~g} / \mathrm{cm}^{3}$, while Lucite was modeled as $1.185 \mathrm{~g} / \mathrm{cm}^{3}$.

1 Lucite is a registered trademark of E. I. duPont de Nemours \& Company

2 Plexiglas is a registered trademark of Rohm \& Haas Company

3 Lexan is a registered trademark of General Electric Company, Chemical Materials Department 


\subsection{DESCRIPTION OF SYSTEM AND FACILITY}

The 234-5Z Building, commonly referred to as PFP (Plutonium Finishing Plant), is located in the 200 West area. This facility was historically used to process plutonium into oxide or metal forms. The facility is undergoing cleanup to stabilize plutonium still stored there.

The plutonium stabilization program at PFP includes conversion of acidic plutonium nitrate solution into plutonium oxide. Conversion is facilitated through use of a vertical calciner installed in Glovebox $\mathrm{HC}-230 \mathrm{C}-2$, which is to be installed in room $230 \mathrm{C}$ of this facility.

\subsection{PROCESS FLOW DESCRIPTION}

Feed material enters as plutonium nitrate solution $\left(\mathrm{Pu}\left(\mathrm{NO}_{3}\right)_{4}\right)$ of at least 1.3M $\mathrm{HNO}_{3}$ or greater (Stubbs, 1997) and is collected in the two feed tanks. This feed material is pumped over to the vertical calciner and heated to an overal1 process temperature of $1000^{\circ} \mathrm{C}$ in excess oxygen/air. A residence time in the calciner of at least 4 hours assists in heating the product to obtain $\mathrm{PuO}_{2}$ with a low loss of weight on ignition (LOI).

Plutonium nitrate suitable for calcination in the vertical calciner is transferred from the $\mathrm{HC}-227 \mathrm{~S}$ glovebox through double encased transfer line to a feed receipt tank located in glovebox $H C-230 \mathrm{C}-2$, room $230 \mathrm{C}$. A controlled volume is then gravity fed into the feed pump tank. Once the feed pump tank contains the appropriate amount of solution, the valve from the feed receipt tank is closed, and the solution in the feed pump tank is mixed with atomizing air flow into a preheated bed of $\mathrm{PuO}_{2}$ powder in the vertical calciner. The vertical calciner agitates the injected mixture between external and internal heaters to $1000^{\circ} \mathrm{C}$ to form plutonium oxide $\left(\mathrm{PuO}_{2}\right)$.

off-gases from this process are removed through the top of the calciner by vacuum. Sintered ceramic filter elements are used to remove any entrained plutonium dioxide powder. The off-gases are then circulated through a scrubber before leaving the glovebox. Spent scrubber solution is staged within the glovebox for sampling before removal from the glovebox. The scrubber system is not expected to contain more than a token amount of fissile

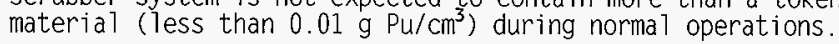

\subsection{GLOVEBOX MODELING FOR SHIELDING CALCULATIONS}

General arrangement of the vertical calciner with the approximate location of the neutron shield wal1 and operator at the electrical control panel are shown in Figure 1. Vertical Calciner Glovebox and Neutron Shield Wa11. Interior details of the Vertical Calciner Glovebox, from hand measurements are illustrated in Figure 2, Vertical Calciner Glovebox Interior Measurements.

Plutonium isotopic compositions were calculated in ORIGEN2 (Croff, 1980; Wittekind. 1994: Schmittroth. 1993) for the gamma ray intensity and energy spectrum and the neutron activity. The neutron activity came from spontaneous fission of, primarily ${ }^{240} \mathrm{Pu}$, and from $(\alpha, n)$ reaction in light elements, primarily oxygen. 
HNF-SD-CP-TI-218 Rev. 0

Fiqure 1. Vertical Calciner Glovebox and Neutron Shield Wall.

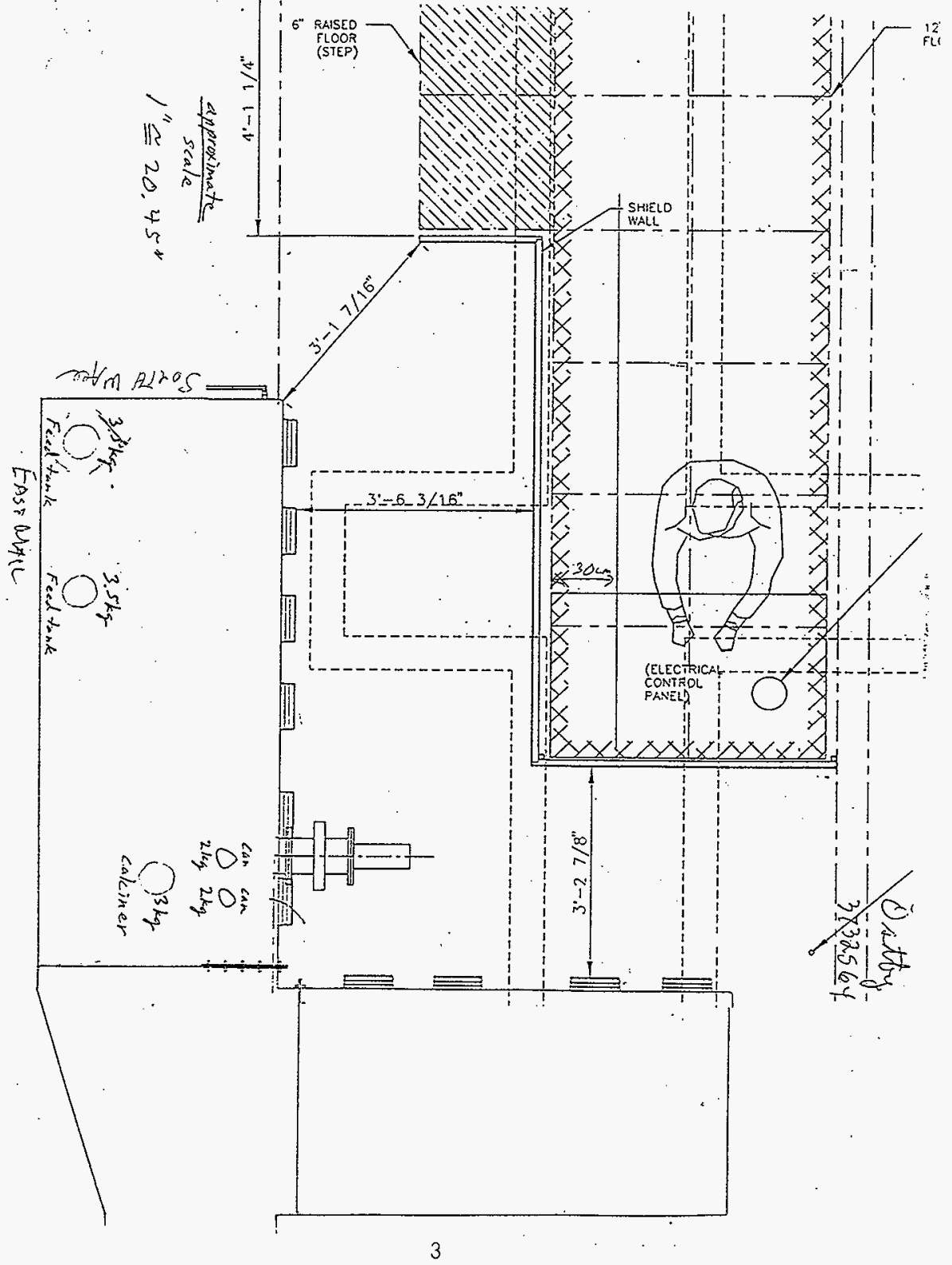


HNF-SD-CP-TI-218 Rev. 0

Figure 2. Vertical Calciner Glovebox Interior Measurements.

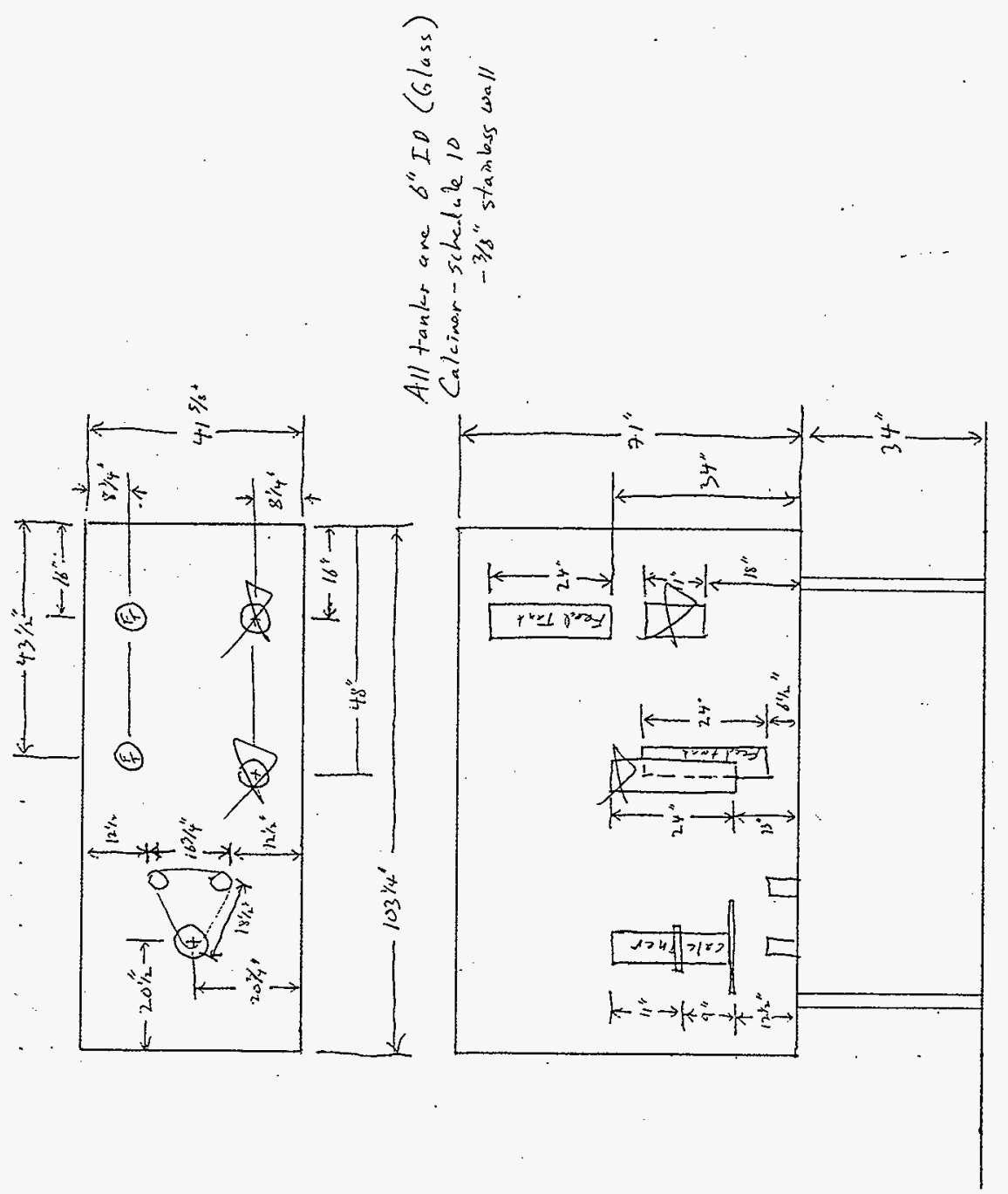


MCNP (Briesmeister, 1993; Carter, 1996) was used to calculate the radiation exposure from the gamma ray source using mode $p$. MCNP was also used to calculate the radiation exposure from the neutron source due to neutrons and gamma rays due to capture gammas using the mode $n$. p. MCNP modeled the vertical calciner glovebox and the entire room with concrete floor, concrete roof, two concrete walls and two metal walls.

The conversion from flux to dose used the ANS 6.1.1 1991 standard with the factor of 2 multiplied times the calculated neutron dose, as specified in ANS 6.1.1 1991. The one exception is that ANS 6.1.1 1977 was used to convert neutron flux to dose for hands around plutonium containers. The ANS 6.1.1 1991 standard with the factor of 2 multiplied times the calculated neutron dose was used for all whole body dose calculations. The ANS 6.1.1 1977 neutron and photon dose conversion factors were reported in $(\mathrm{rem} / \mathrm{hr}) /\left(\mathrm{n} / \mathrm{cm}^{2}-\mathrm{s}\right)$ and were multiplied by $\left(1000\right.$. mrem/rem) to get $(\mathrm{mrem} / \mathrm{hr}) /\left(\mathrm{n} / \mathrm{cm}^{2}-\mathrm{s}\right)$ for use in MCNP.

The ANS 6.1.1 1991 neutron and photon dose conversion factors were reported in $\left(\mathrm{Sv}-\mathrm{cm}^{2}\right)$ and were multiplied by $(3600 . \mathrm{s} / \mathrm{hr}) *(100 . \mathrm{rem} / \mathrm{Sv}) *(1000$. mrem/rem $)$ to get $(\mathrm{mrem} / \mathrm{hr}) /\left(\mathrm{n} / \mathrm{cm}^{2}-\mathrm{s}\right)$ for use in MCNP.

\subsection{CONCENTRATION AND COMPOSITION OF THE FISSILE MATERIAL}

Average inventory plutonium isotopic composition is shown in Table 4.1.

\begin{tabular}{||l|r|r|r||}
\hline \multicolumn{5}{|c|}{ Table 4.1 Average Inventory Isotopic Composition } \\
Plutonium Finishing Plant Stabi lization DE Estimate dated May 16, 1996 \\
\hline Isotope & Low ${ }^{240} \mathrm{Pu} \mathrm{Wt \%}$ & Mid ${ }^{240} \mathrm{Pu}$ Wt\% & High ${ }^{240} \mathrm{Pu}$ Wt\% \\
\hline Pu-238 & 0.01 & 0.15 & 0.18 \\
\hline Pu-239 & 92.40 & 85.63 & 80.06 \\
\hline Pu-240 & 6.12 & 12.45 & 17.84 \\
\hline Pu-241 & 0.26 & 1.20 & 1.22 \\
\hline Pu-242 & 0.04 & 0.37 & 0.73 \\
\hline Am-241 & 0.18 & 1.06 & 1.79 \\
\hline Total Mass (g) & $1,542,620$ & 586,298 & $1,520,313$ \\
\hline
\end{tabular}

\subsubsection{Feed Solution}

The feed tank plutonium inventory assumption of $3.5 \mathrm{~kg}$ plutonium in each 10 Liter tank volume calculates to a concentration of $350 \mathrm{~g} P u / L$ in the plutonium nitrate $\left(\mathrm{Pu}\left(\mathrm{NO}_{3}\right)_{4}\right)$ solution. The density of plutonium nitrate solution is $1.58 \mathrm{~g} / \mathrm{cm}^{3}$. Feed solution at PFP may vary in plutonium concentration in PFP and PUREX solution processes. This $350 \mathrm{~g}$ Pu/L plutonium concentration is conservative based on the material balance flow sheet expected concentration of $250 \mathrm{~g}$ Pu/L (Table 1 in Stubbs, 1997). This $350 \mathrm{~g}$ $\mathrm{Pu} / \mathrm{L}$ plutonium concentration is reasonable based on the plutonium concentration upper limit is the criticality limit (Limit and Control \#2 in Hillesland, 1997$)$ of $450 \mathrm{~g}\left({ }^{239} \mathrm{Pu}\right.$ plus $\left.{ }^{235} \mathrm{U}\right) / \mathrm{L}$. 


\subsubsection{Vertical Calciner Charge}

The vertical denitration calciner is expected (Section 3.4 in Stubbs, $1997)$ to be precharged with approximately $3 \mathrm{~kg}$ of $\mathrm{PuO}_{2}(2.65 \mathrm{~kg} \mathrm{Pu})$ and feed solution is added at $2 \mathrm{~L}$ to $3 \mathrm{~L}$ per hour $(500 \mathrm{~g}$ Pu to $750 \mathrm{~g}$ Pu per hour) and the $\mathrm{PuO}_{2}$ drops out into the receipt can as atomized solution is added into the vertical calciner for normal operating conditions. The assumption of $3.5 \mathrm{~kg}$ $\mathrm{Pu}$ mass in the vertical calciner is reasonable based on this reference.

\subsubsection{Calcine $\left(\mathrm{PuO}_{2}\right)$ Product}

The maximum expected tap density of the calcine product is $5.0 \mathrm{~g} \mathrm{Pu} / \mathrm{cm}^{3}$, or $5.67 \mathrm{~g} \mathrm{PuO} / \mathrm{cm}^{3}$ for normal operating conditions. This is based on experience with the PFP laboratory ${ }_{3}$ calciner, where normal product tap density was found to be 4.0 to $4.3 \mathrm{~g} \mathrm{Pu} / \mathrm{cm}^{3}$. A product density of $4.75 \mathrm{~g} \mathrm{Pu} / \mathrm{cm}^{3}$ was reached when it was continuously heated and stirred without addition of fresh feed solution.

Calcine product average density of $4.5 \mathrm{~g} \mathrm{Pu} / \mathrm{cm}^{3}$ was assumed for evaluation of the radiation dose rate due to plutonium oxide in containers.

\subsection{ROOM 230C MODELING ASSUMPTIONS}

Room $230 \mathrm{C}$ is modeled with:

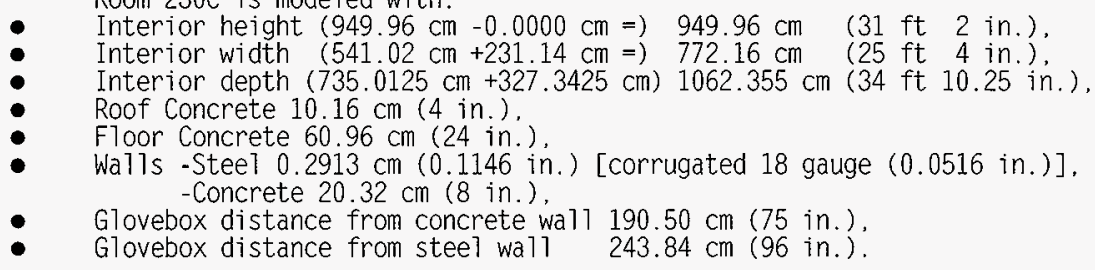

The corrugated steel was estimated to be 4.0 times as long as the distance covered for an effective increase in the 18 gauge thickness from 0.0516 in. to 0.1146 in.

\subsection{GLOVEBOX}

Most of glovebox HC-230C-2 was originally constructed for the Fuels and Materials Examination Facility (FMEF). One portion of the glovebox, calciner section, is primarily used for the calcination process. Another section. waste section, is used to process waste scrubber solution. The third section, connecting section. connects the $\mathrm{HC}-230 \mathrm{C}-2$ glovebox to the $\mathrm{HC}-3$ conveyer for further processing. 
HNF-SD-CP-TI-218 Rev. 0

\subsubsection{Calciner Section of Glovebox}

The calciner section of glovebox is modeled with:

Interior height $(265.7475 \mathrm{~cm}-87.3125 \mathrm{~cm} \Rightarrow 178.435 \mathrm{~cm} \quad(70.25 \mathrm{in}$.$) .$ Interior width $(220.6625 \mathrm{~cm}+39.6875 \mathrm{~cm}=) 260.35 \mathrm{~cm}$ (102.50 in.), Interior depth $(21.2725 \mathrm{~cm}+82.5500 \mathrm{~cm} \Rightarrow 103.8225 \mathrm{~cm}$ (40.875 in.). Walls -Stainless Steel $0.2381 \mathrm{~cm}(3 / 32$ in.). -Lead $0.4763 \mathrm{~cm}(3 / 16 \mathrm{in.})$. -Stainless Steel $0.2381 \mathrm{~cm}(3 / 32$ in.).
- Exterior height $(266.7000 \mathrm{~cm}-86.3600 \mathrm{~cm}=)$

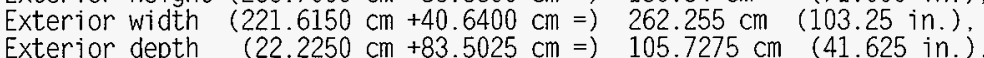
$180.34 \mathrm{~cm} \quad(71.000$ in. $)$. - Glovebox height above concrete floor $86.36 \mathrm{~cm}$ (34.00 in.).

These values are based on scaling from a preliminary drawing and hand measurements. The walls of the glovebox are made from lead sealed between two layers of steel. The lead is $0.476 \mathrm{~cm}(3 / 16 \mathrm{in}$.) thick between the stainless stee] layers. The stainless steel layers are each $0.238 \mathrm{~cm}(3 / 32 \mathrm{in.})$ thick.

The basic model for the calciner section contains the following fissile quantities:

- Both feed tanks filled to 10 Liters of $350 \mathrm{~g} \mathrm{Pu} / \mathrm{L}$ solution is $7.0 \mathrm{~kg} \mathrm{Pu}$.

The calciner contains $3.5 \mathrm{~kg}$ Pu. This section of the calciner is surrounded by $30.48 \mathrm{~cm}$ (12 in.) thick insulation.

- Product receiver can or storage cans may hold $3.6 \mathrm{~kg}$ Pu in a single container. Each container is filled with $4.5 \mathrm{~g} \mathrm{Pu} / \mathrm{cm}^{3}$, dry product. A 4 Liter volume at $4.5 \mathrm{~g} \mathrm{Pu} / \mathrm{cm}^{3}$ dry $\mathrm{PuO}_{2}$ product is $18.0 \mathrm{~kg} \mathrm{Pu}$.

The calciner section of the glovebox, as compared to the waste section and the connection section of the glovebox. contains the bulk of the fissile material for analysis of the entire glovebox. This calciner section is used as the model throughout this analysis.

\subsubsection{Waste Section of Glovebox}

The waste section of the glovebox contains solution used for scrubbing of the off-gasses that come from the calciner. The width of the glovebox (minimum horizontal dimension) is indicated as $107 \mathrm{~cm}$ (42 in.) based on a preliminary drawing with dimensions, and scaling from a second preliminary drawing. The total length of the waste section is only $124.5 \mathrm{~cm}$ (49 in.). The waste section includes two tanks (four cylinders) for storage of spent scrubber solution. These tanks are referred to as spent scrubber receipt tanks (SSRTS).

Because the calciner section of the glovebox contains the bulk of the fissile material of the entire glovebox, the waste section was not modeled in the shielding calculations. 


\subsubsection{Connecting Section of Glovebox}

The connecting section of the glovebox will provide access to one end of the $\mathrm{HC}-3$ conveyer glovebox. Operators wi 11 need to move $0.5 \mathrm{~L}$ slip-lid cans $1.8 \mathrm{~m}(6 \mathrm{ft})$ by hand from the connecting section of the glovebox to the end of the conveyor to be transported to glovebox $\mathrm{HC}-21 \mathrm{~A}$ for and into storage in the Hanford Convenience Can (HCC). The phase separation tank is on the border of this section and the calciner section.

Because the calciner section of the glovebox contains the bulk of the fissile material of the entire glovebox, the connecting section was not modeled in the shielding calculations.

\subsection{FEED SYSTEM}

The feed system for the calciner is mainly located within the calciner portion of the glovebox. The calciner portion of the glovebox includes two feed tanks, a flush tank, feed lines, valves, air supply, and a pump for the feed system. The vent catch tank in the waste section of the glovebox is used to catch overflow from the feed tanks and the flush tank.

\subsubsection{Feed Tanks}

A feed tank has a nominal capacity of $10 \mathrm{~L}$, which means that a feed tank filled with $350 \mathrm{~g} \mathrm{Pu} / \mathrm{cm}^{3}$ feed solution holds a total of $3.5 \mathrm{~kg}$.

Both feed tanks are made from 6 inch Pyrex ${ }^{\$ 4}$ pipe. The feed receipt tank is modeled with:
- Inside radius $7.7610 \mathrm{~cm}$
- $\quad$ Outside radius $8.4534 \mathrm{~cm}$
(3.0555 in.).
(3.3281 in.).

- Pyrex wall thickness $0.6924 \mathrm{~cm}(0.2726$ in.).

Assumes $5 / 16$ in. wall less 0.040 in. tolerance,

Height $(233.6800 \mathrm{~cm}-171.7200 \mathrm{~cm}=) 61.96 \mathrm{~cm}$ (24.3937 in.),

Solution height $(224.5663 \mathrm{~cm}-171.7200 \mathrm{~cm}=) 52.8463 \mathrm{~cm}(20.8056 \mathrm{in}$.),

Distance feed tank centerline to east wal1 $21.2725 \mathrm{~cm}(8.375$ in.).

Distance feed tank centerline to south wa $1739.6875 \mathrm{~cm}(15.625$ in.).

Distance feed tank bottom to glovebox floor

$(171.72 \mathrm{~cm}-87.3125 \mathrm{~cm}=84.4075 \mathrm{~cm}$ (33.2313 in.).

Vendor data indicates an inside diameter of $15.71 \mathrm{~cm}(6.186 \mathrm{in.})$ including tolerances. The height of both feed tanks is nominaily $60.96 \mathrm{~cm}$ (24 in.). The bottom and top of each tank is a $28.575 \mathrm{~cm}$ (11.25 in.) diameter, $1.27 \mathrm{~cm}(1 / 2 \mathrm{in}$.) thick steel flange as measured in the fabrication shop. There is a second flange on both the top and bottom used to hold the pipe in place, which is not included in the model. The feed pump tank was not modeled.

4 Pyrex is a registered trademark of Corning Glass Works 
HNF-SD-CP-TI-218 Rev. 0

The feed pump tank is about $109.5 \mathrm{~cm}$ (43 1/8 in.) from the south wall. and the feed receipt tank about $40.01 \mathrm{~cm}$ ( $153 / 4 \mathrm{in}$.) from the south wa 11 , as determined by scaling from a preliminary drawing and confirmation by hand measurement. The bottom of the feed pump tank internal volume is $16.51 \mathrm{~cm}$ (6 $1 / 2$ in.) from the floor as determined by hand measurement, and the bottom of the interna] volume for the feed receipt tank is $83.32 \mathrm{~cm}$ (33 in.) from the floor as determined by scaling from a preliminary drawing and confirmed by hand measurement. Dose rate from feed receipt tank was multiplied by two that assumes $350 \mathrm{~g} \mathrm{Pu} / \mathrm{L}$ in 10 Liters in both feed tanks.

\subsubsection{Other Feed System Volumes}

A11 pumps in the glovebox have negligible holdup volume for the purpose of this analysis. The waste section of the glovebox contains a vent catch tank, which collects liquid overflow from the feed system. There is a flush tank connected to the feed pump that is used to facilitate restart after unplanned shutdown, and an air supply tank used to atomize feed solution for injection into the calciner. The flush tank has a nominal capacity of only $4 \mathrm{~L}$, the air supply has even less, and neither will contain fissile material under normal conditions.

No other feed system volumes were modeled in the shielding calculations.

\subsection{VERTICAL CALCINER}

The production vertical calciner is virtually identical to the prototype current $7 y$ in the 188-1 glovebox at PFP. Drawing number H-2-95609 (BWHC, 1997) shows dimensions for this new production calciner. The outer vessel of the calciner consists of two sections of 310 stainless steel, 6 inch schedule 10 pipe. The dimensions for this pipe as modeled are $16.15 \mathrm{~cm}(6.357 \mathrm{in}$.) 10 . $16.83 \mathrm{~cm}(6.625 \mathrm{in}$.$) 00. Details specific to each section of pipe are given$ below.

\subsubsection{The Lower Section}

The heating and agitation of the product take place in the lower portion of the calciner. There is a dome made from 4 inch pipe and pipe cap in the center extending up $17.95 \mathrm{~cm}$ (7.066 in.) into the internal volume of the calciner from the bottom. Although the product collection tube will often be filled with product during normal operating conditions, it is of negligible importance for shielding calculations and because it only extends up into the annular region of the calciner.

This section is wrapped in a highly water absorbent insulation. The thickest portion of the insulation is $15.24 \mathrm{~cm}$ ( 6 in.), the insulation is modelled as a close-fitting annulus just over $30.48 \mathrm{~cm}$ (12 in.) thick along its entire axial length. The insulation is $10.16 \mathrm{~cm}$ (4 in.) thick and its inner surface is $9.366 \mathrm{~cm}$ (3.688 in.) from the outside surface of the calciner vessel along most of the middle portion of the insulation's axial length. 
HNF-SD-CP-TI-218 Rev. 0

\subsubsection{The Upper Section}

The upper calcining section contains filter elements for filtering particulates from the off-gasses produced in the calcining process. The filters were modeled based on dimensions from the model used in CSER 95-005 for the calciner in glovebox 188-1 (Geiger. 1995a and 1995b) and a third party reference to personal correspondence with $\mathrm{L}$. $\mathrm{H}$. Rodgers, as there were no drawings made available for these dimensions. These dimensions were used in the base case model for the calciner section of the glovebox as described in Section 4.2.1. Later hand measurements of the production calciner fi]ters by J.F. Durnil yielded different internal dimensions. A list of dimensions for both filter models is given in Table 4.2 below. The inside dimensions are important only for the case of feed solution filling the calciner.

\begin{tabular}{|c|c|c|}
\hline Dimension & $\begin{array}{l}\text { From CSER 95-005 } \\
\text { (Geiger. 1995a) }\end{array}$ & Measurement by J.F. Durni\} \\
\hline Total Length & $30.48 \mathrm{~cm}(12$ in. $)$ & $\begin{array}{l}30.80 \mathrm{~cm}(12 \text { 1/8 in.) } \\
\quad-\text { modeled as } 30.48 \mathrm{~cm}(12 \mathrm{in.})\end{array}$ \\
\hline Outside Diameter & $5.080 \mathrm{~cm}(2$ in. $)$ & $5.080 \mathrm{~cm}$ (2 in.) \\
\hline Inside Diameter & $2.870 \mathrm{~cm}(1.13$ in. $)$ & $3.651 \mathrm{~cm}(17 / 16$ in. $)$ \\
\hline Bottom Thickness & $3.810 \mathrm{~cm}(1.5$ in. $)$ & $2.858 \mathrm{~cm}(11 / 8$ in. $)$ \\
\hline
\end{tabular}

This section is wrapped in metal reflective insulation that does not absorb liquids (see Appendix B of Geiger, 1995b). The manufacturer supplied drawings show an outside diameter of $40 \mathrm{~cm}$ (15.75 in.) and a height of $36.83 \mathrm{~cm}(14.5 \mathrm{in})$ for the prototype calciner. These are the dimensions used for the production calciner. as there was no information available on these dimensions at the time of the analysis. The insulation was modeled as being $10 \%$ density $304 \mathrm{~L}$ steel to accommodate the concentric sheets and narrow strips of sheet metal used to create many spaces of dead air within the insulation. In any case, the amount of steel modeled is small.

\subsubsection{Product Collection Tube}

A product collection tube is used to feed the final product from the calciner into product receipt cans. The outside pipe of the tube is 1 in. schedule 10s pipe. The top of the tube is inside the center dome of the calciner. There is a $11.43 \mathrm{~cm}$ (4.5 in.) tall slot in the side of the dome. and a slot cut into the side of the tube to allow product to flow from the calciner down the tube into an attached product receipt can. The height of the product inside the calciner is controlled by a weir inside the tube. The flow of product is controlled by a valve attached to the product collection tube. The tube was found to adjust such that the bottom may range between $12.7 \mathrm{~cm}$ ( 5 in.) to $30.48 \mathrm{~cm}$ (12 in.) from the glovebox floor based on hand measurement in the fabrication shop. 
HNF-SD-CP-TI-218 Rev. 0

\subsection{CONTAINERS}

There are three types of plutonium oxide containers that will be allowed in the glovebox: product receiver vessels, $0.5 \mathrm{~L}$ containers (1 pound slip lid cans and polyjars), and $30 \mathrm{~mL}$ sample jars. A limit requires that no more than two unit masses of $2 \mathrm{~L}$ each be in the glovebox at one time.

Two receipt cans are considered: a $3.6 \mathrm{~kg} \mathrm{Pu}$ can, and a $1.8 \mathrm{~kg} \mathrm{Pu}$ can. The dimensions of the $3.6 \mathrm{~kg}$ Pu receipt can are:

$4.6038 \mathrm{~cm}$ (1.8125 in. or $113 / 16$ in.) Inside Radius

$4.7625 \mathrm{~cm}$ (1.8750 in. or $17 / 8$ in.) Outside Radius

$11.2713 \mathrm{~cm}(4.4375$ in. or $47 / 16$ in.) Inside Height

$11.43 \mathrm{~cm}$ (4.5 in. or $41 / 2$ in.) Outside Height

The dimensions of the $1.8 \mathrm{~kg}$ Pu receipt can are:

$3.8894 \mathrm{~cm}$ (1.53125 in. or $117 / 32 \mathrm{in.})$ Inside Radius

$4.0481 \mathrm{~cm}$ ( 1.5937 in. or $119 / 32$ in.) Outside Radius

$7.8582 \mathrm{~cm}(3.0938 \mathrm{in}$. or $33 / 32 \mathrm{in.})$ Inside Height

$8.0169 \mathrm{~cm}$ (3.1563 in. or $35 / 32$ in.) Outside Height

The $0.5 \mathrm{~L}$ (nominal) containers proposed for use in the $\mathrm{HC}-230 \mathrm{C}-2$ glovebox are the 1 pound slip 7 id can (part number 42-1500-300) with the dimensions of $8.89 \mathrm{~cm}(3.5 \mathrm{in.})$ oD $\times 8.89 \mathrm{~cm}(3.5 \mathrm{in.})$ height, and the polyjar (part number 57-6359-160) with the dimensions of $8.255 \mathrm{~cm}(3.25 \mathrm{in}$.$) OD \mathrm{x}$ $10.16 \mathrm{~cm}$ (4 in.) height. The total internal volume for the 1 pound slip lid can is $552 \mathrm{~mL}$, and for the polyjar it is $543 \mathrm{~mL}$.

A unit mass is considered to be 2 Liters. There are permitted to be two unit masses in containers inside the vertical calciner glovebox at the same time. This is a total volume of 4 Liters of $\mathrm{PuO}_{2}$. Because of the assumed density of $4.5 \mathrm{~g} \mathrm{Pu} / \mathrm{L}$, this equates to $4 \mathrm{~L} * 4.5^{2} \mathrm{~kg} \mathrm{Pu} / \mathrm{L}=18 \mathrm{~kg}$ Pu total. The dose calculation is performed for $3.6 \mathrm{~kg}$ Pu per receipt can, so the contribution to the total radiation dose rate from $\mathrm{PuO}_{2}$ in containers is caiculated for five of these $3.6 \mathrm{~kg}$ Pu receipt cans.

\subsection{SCRUBBER SYSTEM}

The scrubber system is not expected to contain more than a token amount of fissile material (less than $0.01 \mathrm{~g} \mathrm{Pu} / \mathrm{cm}^{3}$ ) during normal operations. No scrubber system volumes were modeled in the shielding calculations.

\subsection{EVALUATION AND RESULTS}

\subsection{PLUTONIUM INVENTORY OF VERTICAL CALCINER GLOVEBOX}

The vertical calciner glovebox plutonium inventory is assumed to be:

- $\quad 7.0 \mathrm{~kg}$ plutonium in Pu(NO$\left.)_{3}\right)_{4}$ solution in the two 10 Liter feed tanks,

- $\quad 3.5 \mathrm{~kg}$ plutonium in $\mathrm{PuO}_{2} \mathrm{in}^{4}$ the vertical calciner, and

$18.0 \mathrm{~kg}$ plutonium in $\mathrm{PuO}_{2}$ in 4 Liter total volume of containers.

There is sufficient plutonium assumed in these separate components to compensate for the plutonium solution in the piping into the feed tank in the glovebox, and between the feed tanks and the vertical calciner. as well as the possibility that there may be spilled plutonium on the glovebox floor. 
The feed tank plutonium inventory assumption of $3.5 \mathrm{~kg}$ plutonium in each 10 Liter tank volume calculates to a concentration of $350 \mathrm{~g} \mathrm{Pu} / \mathrm{L}$ in the plutonium nitrate $\left(\mathrm{Pu}\left(\mathrm{NO}_{3}\right)_{4}\right)$ solution. This plutonium concentration is conservative based on the material balance flow sheet assumption (Table 1 in Stubbs, 1997) of $250 \mathrm{~g} \mathrm{Pu} / \mathrm{L}$. This plutonium concentration is reasonable based on the plutonium concentration upper limit is the criticality limit (Limit and Control \#2 in Hillesiand, 1997) of $450 \mathrm{~g}\left({ }^{239} \mathrm{Pu}\right.$ plus $\left.{ }^{235} \mathrm{U}\right) / \mathrm{L}$.

The vertical calciner plutonium inventory assumption is $3.5 \mathrm{~kg}$ plutonium. This plutonium inventory in the vertical calciner is reasonable based on the material balance flow sheet assumption of $3.0 \mathrm{~kg}$ plutonium for calciner bed holdup (Table 1 in Stubbs, 1997).

The container plutonium inventory in receipt cans (two at 1 Liter each) and storage cans (four slip lid cans at 0.5 Liters each) assumption of $18 \mathrm{~kg}$ plutonium in a total volume of 4 Liters calculates to a density of $4.5 \mathrm{~g} \mathrm{Pu} / \mathrm{cm}^{3}$. Normal tap density for a PFP' laboratory calciner was reported to be $4.0 \mathrm{~g} \mathrm{Pu} / \mathrm{cm}^{3}$ to $4.3 \mathrm{~g} \mathrm{Pu} / \mathrm{cm}^{3}$ (Page 5 in Hillesland, 1997). This plutonium density is reasonable based on the $3.6 \mathrm{~g} \mathrm{PuO} / \mathrm{cm}^{3}$ highest observed calcined plutonium density starting with plutonium nitrate (Page II.C.2-2 in ARC-600). The plutonium density upper limit is the criticality limit (Limit and Control \#1 in Hillesland, 1997) of $5.5 \mathrm{~g} \mathrm{Pu} / \mathrm{cm}^{3}$.

\subsection{PLUTONIUM ACTIVITY CALCULATION BY ORIGEN2}

This radiation shielding calculation began with the isotopic compositions of the plutonium in the calciner feed stock. An ORIGEN2 calculation converted the plutonium into gamma ray intensity and energy spectrum, as well as a spontaneous neutron activity and an $(\alpha, n)$ neutron activity. The gamma ray intensity and energy spectrum were used for the source term in MCNP in mode $p$ to calculate the direct gamma ray flux. The neutron activity was used for the source term in MCNP in mode $n, p$ to calculate the neutron flux and the associated $(n, v)$ induced gamma ray flux.

The ORIGEN2 input file as well as calculated gamma ray energy spectrum and neutron sources are listed in Appendix B. ORIGEN2 Plutonium Input File and Output Results.

\subsection{COMPONENT DOSE RATE CALCULATION BY MCNP}

The conversion from flux to dose used the ANS 6.1.1 1991 standard. The photon flux to dose rate conversion was used directly, but the neutron flux to dose rate conversion was multiplied by the factor of 2 , as specified in ANS 6.1.1 1991. The total radiation dose rate from a single plutonium source is the sum of these individual gamma ray. neutron, and induced gamma ray dose rate contributions. The total calciner glovebox radiation dose rate expected from the calciner glovebox is the sum of contributions from plutonium in a11 the individual sources within the glovebox such as the feed tank, the vertical calciner, and the storage cans. There are two feed tanks, one is the feed tank (receipt), and the other is the feed tank (pump), but both are the same 10 Liter volume. The vertical calciner is considered for its plutonium inventory. The plutonium oxide in storage cans without the shielding of the feed tank or the vertical calciner contributes more to the radiation dose rate for the same quantity of plutonium. 
The plutonium mass assumed $350 \mathrm{~g} \mathrm{Pu} / \mathrm{L}$ in the $\mathrm{Pu}\left(\mathrm{NO}_{3}\right)_{4}$ solution with a total of 10 Liters in both the feed tank (receipt) and feed tank (pump). The plutonium mass assumed in the calciner is $3.5 \mathrm{~kg} \mathrm{Pu}$. The container plutonium mass assumed $4.5 \mathrm{~g} \mathrm{Pu} / \mathrm{cm}^{3}$ in the $\mathrm{PuO}_{2}$ compound in the two $1 \mathrm{~L}$ receipt cans and in four $0.5 \mathrm{~L}$ slip lid cans (total $4.0 \mathrm{~L}$ in cans). This quantities yielded a total of $7.0 \mathrm{~kg} \mathrm{Pu}$ in the feed tanks. $3.5 \mathrm{~kg}$ Pu in the calciner, and $18.0 \mathrm{~kg}$ $\mathrm{Pu}$ in cans as the source term for the calciner glovebox.

\subsection{EFFECTIVE DOSE RATE CALCULATION BY WEIGHTING}

Operator dose rate is added from gamma, capture gamma, and neutron dose contributions from feed tanks, vertical calciner, and plutonium oxide collected in cans. Annual dose rates were scaled proportionately for the three categories of ${ }^{240} \mathrm{Pu}$ material.

Table 5.1 neutron shield wa11 design with lower $137.16 \mathrm{~cm}$ (4.5 feet) of $12.7 \mathrm{~cm}$ ( 5 in.) thick Lucite ${ }^{\circledR}$ and $0.3175 \mathrm{~cm}(0.125$ in.) stainless steel on both sides, and upper $76.20 \mathrm{~cm}\left(2.5\right.$ feet) of $10.16 \mathrm{~cm}$ (4 in.) thick Lucite ${ }^{\circledR}$ and $1.905 \mathrm{~cm}(0.75 \mathrm{in}$.) thick glass on each side gave a total dose rates of

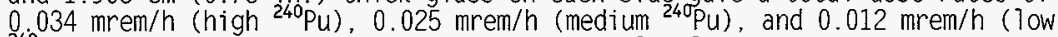
${ }^{240} \mathrm{Pu}$ ) for $3.5 \mathrm{~kg}$ plutonium inside the vertical calciner.

\begin{tabular}{|c|c|c|c|c|}
\hline \multicolumn{5}{|c|}{$\begin{array}{c}\text { Table } 5.1 \text { Lucite }{ }^{\circledR} \text { Neutron Shield } \\
\text { Bottom } 4.5 \mathrm{ft} \text {. is } 5 \text { in. Lucite } e^{\circledR} \text { and } 1 / 8 \text { in. SST Both Sides } \\
\text { Top } 2.5 \mathrm{ft} \text {. is } 4 \text { in. Lucite }{ }^{\circledR} \text { and } 3 / 4 \text { in. GTass Both Sides } \\
\text { Source Term used High }{ }^{240} \mathrm{Pu} \text { Category Plutonium } \\
0.23 \mathrm{mrem} / \mathrm{h}=1 \times \text { Calciner }+2 \times \text { Feed Tank }+5 \times \text { Storage Can }\end{array}$} \\
\hline \multicolumn{5}{|c|}{ Calciner with $3.5 \mathrm{~kg}$ Plutonium - Dose Rates behind Neutron Shield (mrem $/ \mathrm{h})$} \\
\hline cca08anp.out & & High Pu-240 & Mid Pu-240 & Low Pu-240 \\
\hline $3.13090 \mathrm{E}-02 \quad 0.0680$ & Neutron & $3.13090 \mathrm{e}-02$ & $2.28184 \mathrm{e}-02$ & $1.11439 \mathrm{e}-02$ \\
\hline $1.03211 \mathrm{E}-03 \quad 0.0648$ & Gamma $(n, \gamma)$ & $1.03211 \mathrm{e}-03$ & $7.52216 \mathrm{e}-04$ & $3.67361 \mathrm{e}-04$ \\
\hline $1.68063 \mathrm{E}-03 \quad 0.3923$ & Gamma & 1.680 & $1.11423 \mathrm{e}-03$ & 3.165 \\
\hline \begin{tabular}{|l|l|}
0.229874 & 0.023004 \\
\end{tabular} & Total & $3.40217 \mathrm{e}-02$ & $2.46849 \mathrm{e}-02$ & $1.18278 \mathrm{e}-02$ \\
\hline \multicolumn{5}{|c|}{ Feed Tank with $3.5 \mathrm{~kg}$ Plutonium - Dose Rates behind Neutron Shield (mrem $/ \mathrm{h}$ ) } \\
\hline cft08anp. out & & High Pu-240 & Mid Pu-240 & Low Pu-240 \\
\hline $3.47302 E-02 \quad 0.0216$ & Neutron & $3.47302 \mathrm{e}-02$ & $2.53118 \mathrm{e}-02$ & 1.236 \\
\hline $1.90412 \mathrm{E}-03 \quad 0.0183$ & Gamma $(n, y)$ & $1.90412 \mathrm{e}-03$ & $1.38775 \mathrm{e}-03$ & 6.777 \\
\hline $6.89650 \mathrm{E}-03 \quad 0.0799$ & Gamma & 6.896 & $4.57228 \mathrm{e}-03$ & $1.29917 \mathrm{e}-03$ \\
\hline \begin{tabular}{l|l|} 
& 0.02905 \\
\end{tabular} & Total & $4.35308 \mathrm{e}-02$ & $3.12719 \mathrm{e}-02$ & $1.43385 \mathrm{e}-02$ \\
\hline \multicolumn{5}{|c|}{ Storage Can's $3.6 \mathrm{~kg}$ Plutonium - Dose Rates behind Neutron Shield (mrem/h) } \\
\hline csco8anp.out & & High Pu-240 & Mid Pu-240 & Low Pu-240 \\
\hline $4.10801 E-02 \quad 0.0602$ & Neutron & $4.10801 \mathrm{e}-02$ & $2.99397 \mathrm{e}-02$ & $1.46217 \mathrm{e}-02$ \\
\hline $1.44262 \mathrm{E}-03 \quad 0.0537$ & Gamma $(n, \gamma)$ & $1.44262 \mathrm{e}-03$ & $1.05140 \mathrm{e}-03$ & $5.13475 \mathrm{e}-04$ \\
\hline $1.38752 E-03 \quad 0.2774$ & Gamma & $1.38752 \mathrm{e}-03$ & 9.19907e-04 & $2.61383 \mathrm{e}-04$ \\
\hline \begin{tabular}{l|l|} 
& 0.029754 \\
\end{tabular} & Total & $4.39102 \mathrm{e}-02$ & $3.19110 \mathrm{e}-02$ & $1.53966 \mathrm{e}-02$ \\
\hline
\end{tabular}


Weighting an annual dose rate proportional to plutonium quantities in the different ${ }^{240} \mathrm{Pu} w \mathrm{w} \%$ categories for an average radiation dose rate is:

$(0.034 * 1,542,620 .+0.025 * 586,298 .+0.012 * 1,520,313) / 3,649,.231 .=0.023 \mathrm{mrem} / \mathrm{h}$

for $3.5 \mathrm{~kg}$ plutonium in the vertical calciner. Similar calculations are performed in Table 5.1 for $3.5 \mathrm{~kg}$ plutonium in the feed tank, and for $3.6 \mathrm{~kg}$ plutonium in storage cans. Adding:

$1 *($ vertical calciner $)+2 *($ feed tank $)+5 *($ storage cans $)=$ average $\mathrm{mrem} / \mathrm{h}$ $1 *(0.023 \mathrm{mrem} / \mathrm{h})+2 *(0.029 \mathrm{mrem} / \mathrm{h})+5^{*}(0.030 \mathrm{mrem} / \mathrm{h})=0.23 \mathrm{mrem} / \mathrm{h}$

satisfying the $0.25 \mathrm{mrem} / \mathrm{h}$ design limit, fulfilling the design goal.

\subsection{COMPARISON OF LUCITE AND LEXAN NEUTRON SHIELDING MATERIAL}

Table 5.2 neutron shield wa7l design with lower $41 / 2$ feet of 5 inches thick Lexan and $1 / 8$ inch stainless steel on both sides, and upper $21 / 2$ feet of 4 inches thick Lexan ${ }^{\circledR}$ and $3 / 4$ inches thick glass on each side gave a total weighted dose rate of $0.30 \mathrm{mrem} / \mathrm{h}$, exceeding the design goal.

\begin{tabular}{|c|c|c|c|c|}
\hline $\begin{array}{r}\text { Bottom } 4.5 \\
\text { Top } 2.5 \mathrm{ft} \text {. } \\
\text { Source } \\
0.30 \mathrm{mrem} / \\
\end{array}$ & $\begin{array}{l}\text { Table } 5.2 \text { Le } \\
\text { is } 5 \text { in. Le } \\
\text { is } 4 \text { in. Lexa } \\
\text { Term used Hio } \\
=1 \times \text { Calciner }\end{array}$ & $\begin{array}{l}\operatorname{ran}^{\circledast} \text { Neutron } \mathrm{S} \\
\text { xan and } 1 / 8 \\
{ }^{\circledR} \text { and } 3 / 4 \text { in } \\
h^{240} \mathrm{Pu} \text { Catego } \\
+ \text { 2xFeed Tank } \\
\end{array}$ & $\begin{array}{l}\text { hield } \\
\text { n. SST Both S } \\
\text { Glass Both Si } \\
\text { y Plutonium } \\
+ \text { 5xStorage } 0 \\
\end{array}$ & \\
\hline Calciner with $3.5 \mathrm{~kg}$ & lutonium - D & se Rates behir & Id Neutron Shi & id (mre \\
\hline cca08bno. out & & High Pu-240 & Mid Pu-240 & Low Pu-240 \\
\hline $3.92718 \mathrm{E}-02 \quad 0.0640$ & Neutron & $3.92718 \mathrm{e}-02$ & $2.86218 \mathrm{e}-02$ & $1.39781 \mathrm{e}-02$ \\
\hline $8.50756 \mathrm{E}-04 \quad 0.0696$ & Gamma $(n, y)$ & $8.50756 \mathrm{e}-04$ & $6.20043 \mathrm{e}-04$ & $3.02811 \mathrm{e}-04$ \\
\hline $1.90409 \mathrm{E}-03 \quad 0.3617$ & Gamma & $1.90409 \mathrm{e}-03$ & $1.26239 \mathrm{e}-03$ & $3.58695 \mathrm{e}-0.4$ \\
\hline \begin{tabular}{l|l}
0.295817 & 0.028431 \\
\end{tabular} & Total & $4.20266 \mathrm{e}-02$ & $3.05042 \mathrm{e}-02$ & $1.46396 \mathrm{e}-02$ \\
\hline Feed Tank with $3.5 \mathrm{~kg}$ & Prutonium - & ose Rates beh & nd Neutron Sh & eld (mrem/h) \\
\hline cft08bnp.out & & High Pu-240 & Mid Pu-240 & Low Pu-240 \\
\hline $4.26543 \mathrm{E}-02 \quad 0.0194$ & Neutron & $4.26543 \mathrm{e}-02$ & $3.10870 \mathrm{e}-02$ & $1.51820 \mathrm{e}-02$ \\
\hline $1.79059 \mathrm{E}-03 \quad 0.0191$ & $\operatorname{Gamma}(n, y)$ & $1.79059 \mathrm{e}-03$ & $1.30501 \mathrm{e}-03$ & $6.37329 e-04$ \\
\hline $\begin{array}{ll}7.12845 \mathrm{E}-03 & 0.0729 \\
\end{array}$ & Gamma & $7.12845 \mathrm{e}-03$ & $4.72606 \mathrm{e}-03$ & $1.34287 \mathrm{e}-03$ \\
\hline \begin{tabular}{l|l} 
& 0.034501 \\
\end{tabular} & Total & $5.15733 \mathrm{e}-02$ & $3.71181 \mathrm{e}-02$ & $1.71622 \mathrm{e}-02$ \\
\hline Storage Can's $3.6 \mathrm{~kg}$ & itonium - D & se Rates behi & d Neutron Shi & ld $(\mathrm{mrem} / \mathrm{h})$ \\
\hline csc08bnp.out & & High Pu-240 & Mid Pu-240 & Low Pu-240 \\
\hline $5.58616 \mathrm{E}-02 \quad 0.0513$ & Neutron & $5.58616 \mathrm{e}-02$ & $4.07127 e-02$ & $1.98829 \mathrm{e}-02$ \\
\hline $1.30592 \mathrm{E}-03 \quad 0.0590$ & Gamma $(n, y)$ & $1.30592 \mathrm{e}-03$ & $9.51772 \mathrm{e}-04$ & $4.64819 \mathrm{e}-04$ \\
\hline $1.32357 \mathrm{E}-03 \quad 0.2906$ & Gamma & $1.32357 \mathrm{e}-03$ & $8.77509 \mathrm{e}-04$ & $2.49336 \mathrm{e}-04$ \\
\hline \begin{tabular}{l|l} 
& 0.039677 \\
\end{tabular} & Total & $5.84911 \mathrm{e}-02$ & $4.25420 \mathrm{e}-02$ & $2.05971 \mathrm{e}-02$ \\
\hline
\end{tabular}


The Lucite ${ }^{\circledR}$ was modeled as methyl methacrylate $\left(\mathrm{C}_{5} \mathrm{H}_{8} \mathrm{O}_{2}\right.$ at $\left.1.185 \mathrm{~g} / \mathrm{cm}^{3}\right)$, while the Lexan ${ }^{\oplus}$ was modeled as polycarbonate $\left(\mathrm{C}_{16} \mathrm{H}_{14} \mathrm{O}_{3}\right.$ at $\left.{ }^{2}{ }^{2} .2 \mathrm{~g} / \mathrm{cm}^{3}\right)$ (Table 9.2-33 in Jaeger et. a1. 1975).

\subsection{COMPENSATION FOR INCREASED $(\alpha, n)$ IN PLUTONIUM NITRATE SOLUTION}

The $(\alpha, n)$ activity for plutonium is calculated in ORIGEN2 according to the plutonium being plutonium oxide $\left(\mathrm{PuO}_{2}\right)$, while the plutonium in the feed tanks is actually in $\mathrm{Pu}\left(\mathrm{NO}_{3}\right)_{4}$ in aqueous solution. The $(\alpha, n)$ reaction could be twice as large in the solution as the $(\alpha, n)$ activity in $\mathrm{PuO}_{2}$.

\begin{tabular}{|c|c|c|c|}
\hline \multicolumn{4}{|c|}{$\begin{array}{l}\text { Table } 5.3 \text { Increased }(\alpha, n) \text { Effect } \\
\text { Effect of Increased }(\alpha, n) \text { Yield in Plutonium } \\
\text { Nitrate Solution Compared to Plutonium Oxide }\end{array}$} \\
\hline & Low ${ }^{240} \mathrm{Pu}$ & Medium ${ }^{240} \mathrm{Pu}$ & $\mathrm{High}{ }^{240} \mathrm{Pu}$ \\
\hline \multicolumn{4}{|c|}{$(\alpha, N)$ NEUTRON SOURCE $(n / \mathrm{sec})$} \\
\hline TABLE & 53.44 & 104.6 & 134.6 \\
\hline \multicolumn{4}{|c|}{ SPONTANEOUS FISSION NEUTRON SOURCE $(\mathrm{n} / \mathrm{sec})$} \\
\hline TABLE & 50.5 & 108.1 & 155.6 \\
\hline \multicolumn{4}{|c|}{ OVERALL TOTAL } \\
\hline TOTAL & 103.9 & 212.7 & 290.2 \\
\hline \multicolumn{4}{|c|}{ 2. $0 *(\alpha, n)$ NEUTRON SOURCE $(n / \mathrm{sec})$} \\
\hline TABLE & 106.88 & 209.2 & 269.2 \\
\hline \multicolumn{4}{|c|}{ SPONTANEOUS FISSION NEUTRON SOURCE $(n / \mathrm{sec})$} \\
\hline TABLE & 50.5 & 108.1 & 155.6 \\
\hline \multicolumn{4}{|c|}{ OVERALL TOTAL } \\
\hline TOTAL & 157.38 & 317.3 & 424.8 \\
\hline \multicolumn{4}{|c|}{$\begin{array}{l}\text { RATIO OF TOTAL NEUTRON SOURCE WITH }(\alpha, n) \text { DOUBLED } \\
\text { TO ORIGINAL ORIGEN2 NEUTRON SOURCE }\end{array}$} \\
\hline & 1.514726 & 1.491772 & 1.463818 \\
\hline
\end{tabular}




\begin{tabular}{|c|c|c|c|c|}
\hline \multicolumn{5}{|c|}{ 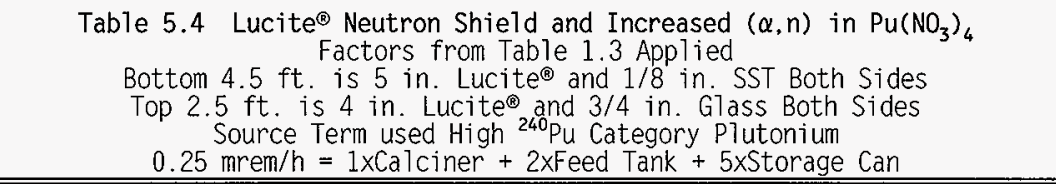 } \\
\hline \multicolumn{5}{|c|}{ Calciner with $3.5 \mathrm{~kg}$ Plutonium - Dose Rates behind Neutron Shield (mrem/h) } \\
\hline cca08anp.out & & High Pu-240 & Mid Pu-240 & Low Pu-240 \\
\hline $3.13090 \mathrm{E}-02 \quad 0.0680$ & Neutron & $3.13090 \mathrm{e}-02$ & $2.28184 \mathrm{e}-02$ & $1.11439 \mathrm{e}-02$ \\
\hline $1.03211 \mathrm{E}-03 \quad 0.0648$ & Gamma $(n, y)$ & $1.03211 \mathrm{e}-03$ & $7.52216 \mathrm{e}-04$ & $3.67361 \mathrm{e}-04$ \\
\hline $1.68063 \mathrm{E}-03 \quad 0.3923$ & Gamma & $1.68063 \mathrm{e}-03$ & $1.11423 \mathrm{e}-03$ & $3.16599 \mathrm{e}-04$ \\
\hline 0.023004 & Total & $3.40217 \mathrm{e}-02$ & $2.46849 \mathrm{e}-02$ & $1.18278 \mathrm{e}-02$ \\
\hline \multicolumn{5}{|c|}{ Feed Tank with $3.5 \mathrm{~kg}$ Plutonium - Dose Rates behind Neutron Shield (mrem/h) } \\
\hline cft08anp.out & & High Pu-240 & Mid Pu-240 & Low Pu-240 \\
\hline $3.47302 E-02 \quad 0.0216$ & Neutron & $3.47302 \mathrm{e}-02$ & $2.53118 \mathrm{e}-02$ & $1.23616 \mathrm{e}-02$ \\
\hline $1.90412 \mathrm{E}-03 \quad 0.0183$ & Gamma $(n, y)$ & $1.90412 \mathrm{e}-03$ & $1.38775 \mathrm{e}-03$ & $6.77738 \mathrm{e}-04$ \\
\hline $6.89650 \mathrm{E}-03 \quad 0.0799$ & Gamma & $6.89650 \mathrm{e}-03$ & $4.57228 \mathrm{e}-03$ & $1.29917 \mathrm{e}-03$ \\
\hline 0.041003 & Total & $6.05225 \mathrm{e}-02$ & $4.44020 \mathrm{e}-02$ & $2.10502 \mathrm{e}-02$ \\
\hline \multicolumn{5}{|c|}{ Storage Can's $3.6 \mathrm{~kg}$ Plutonium - Dose Rates behind Neutron Shield (mrem/h) } \\
\hline csco8anp.out & & High Pu-240 & Mid Pu-240 & Low Pu-240 \\
\hline $4.10801 E-02 \quad 0.0602$ & Neutron & $4.10801 e-02$ & $2.99397 \mathrm{e}-02$ & $1.46217 \mathrm{e}-02$ \\
\hline $1.44262 \mathrm{E}-03 \quad 0.0537$ & Gamma $(n, y)$ & $1.44262 \mathrm{e}-03$ & $1.05140 \mathrm{e}-03$ & $5.13475 \mathrm{e}-04$ \\
\hline $1.38752 \mathrm{E}-03 \quad 0.2774$ & Gamma & $1.38752 \mathrm{e}-03$ & $9.19907 e-04$ & $2.61383 e-04$ \\
\hline 0.029754 & Total & $4.39102 \mathrm{e}-02$ & $3.19110 \mathrm{e}-02$ & $1.53966 \mathrm{e}-02$ \\
\hline
\end{tabular}

\subsection{DOSE RATE PATHWAYS TO OPERATOR BEHIND NEUTRON SHIELD}

The dose rate pathways were calculated by using MCNP to flag tallies that arrived at the cell behind the neutron shield after having traversed specified cells that completely surround the operator. The results are tabulated in Table 5.5, Radiation Dose Rate From The Different Pathways. These results can be tabulated as follows:
Plutonium Source:
Calciner
Feed Tank
Containers
Dominant Radiation:
Neutron
Neutron
Neutron
Principal Pathway(s):
$F-L$ n Shield, \&
$F-L=F r o n t$ Lower
S-L n Shield.
Open End, \&
F-L n Shield, \&
$S-L=S i d e$ Lower, etc.
F-U n Shield, \&
$F-L$ n Shield. 


\begin{tabular}{|c|c|c|c|}
\hline \multicolumn{4}{|c|}{$\begin{array}{l}\text { Table } 5.5 \text { Radiation Dose Rate From The Different Pathways } \\
\text { Upper n Shield: Lucite } 4 \text { inches, 3/4" Glass Both Sides } \\
\text { Lower n Shield: Lucite } 5 \text { inches, 1/8" Stainless Steel Both Sides } \\
\text { End n Shield: Lucite } 1 \text { inch, Glass on Upper, SST on Lower } \\
\end{array}$} \\
\hline $\begin{array}{l}\text { Radiation Leakage or } \\
\text { Reflection Path }\end{array}$ & Neutron $\mathrm{mrem} / \mathrm{h}$ & Gamma $(\mathrm{n}, \mathrm{y}) \mathrm{mrem} / \mathrm{h}$ & Gamma mrem/h \\
\hline $3.5 \mathrm{~kg}$ Pu-Calciner & \multicolumn{2}{|l|}{ cca08anp.out } & cca08ap . out \\
\hline Open End Leakage & $3.58199 \mathrm{E}-030.1537$ & $9.99946 \mathrm{E}-05 \quad 0.2490$ & $1.81439 \mathrm{E}-07 \quad 1.0000$ \\
\hline Front Lower Leakage & $8.89764 \mathrm{E}-03 \quad 0.1281$ & $6.83875 \mathrm{E}-04 \quad 0.0802$ & $1.53766 \mathrm{E}-03 \quad 0.4263$ \\
\hline Front Upper Leakage & $\begin{array}{llll}4.46445 \mathrm{E}-03 & 0.1578 \\
\end{array}$ & $2.71602 \mathrm{E}-04 \quad 0.1326$ & $1.42788 \mathrm{E}-04 \quad 0.4964$ \\
\hline Side Lower Leakage & $9.02347 \mathrm{E}-03 \quad 0.1349$ & $1.05918 \mathrm{E}-04 \quad 0.2128$ & $1.23192 \mathrm{E}-05 \quad 1.0000$ \\
\hline Side Upper Leakage & $2.61693 E-03 \quad 0.2266$ & $4.47746 \mathrm{E}-05 \quad 0.3100$ & $858 \mathrm{E}-06 \quad 1.0000$ \\
\hline Roof Reflection & $6.58581 \mathrm{E}-03 \quad 0.1427$ & $6.59565 \mathrm{E}-05 \quad 0.3428$ & $0.00000 E+00 \quad 0.0000$ \\
\hline Steel Wall Reflect & $3.60208 \mathrm{E}-04 \quad 0.8342$ & $1.52006 \mathrm{E}-06 \quad 0.8779$ & $0.00000 \mathrm{E}+00 \quad 0.0000$ \\
\hline Concrete Floor Effect & $1.02145 \mathrm{E}-02 \quad 0.1137$ & $4.22720 \mathrm{E}-04 \quad 0.1016$ & $7.52464 \mathrm{E}-05 \quad 0.8600$ \\
\hline Total $\mathrm{mrem} / \mathrm{h}$ & $3.13090 \mathrm{E}-02 \quad 0.0680$ & $1.03211 \mathrm{E}-03 \quad 0.0648$ & $1.68063 \mathrm{E}-03 \quad 0.3923$ \\
\hline $3.5 \mathrm{kq}$ Pu-Feed Tank & \multicolumn{2}{|c|}{ cft08anp. out } & 3ap.out \\
\hline Open End Leakage & $1.15342 \mathrm{E}-02 \quad 0.0383$ & $1.68144 \mathrm{E}-04 \quad 0.0573$ & $3.61090 \mathrm{E}-04 \quad 0.2958$ \\
\hline Front Lower Leakage & $8.53409 E-03 \quad 0.0427$ & $1.05428 \mathrm{E}-03 \quad 0.0248$ & $2.75062 \mathrm{E}-03 \quad 0.1286$ \\
\hline Front Upper Leakage & $1.04826 \mathrm{E}-02 \quad 0.0393$ & $7.24873 \mathrm{E}-04 \quad 0.0290$ & $3.70877 \mathrm{E}-03 \quad 0.1096$ \\
\hline Side Lower Leakage & $2.22065 \mathrm{E}-03 \quad 0.0865$ & $7.95798 \mathrm{E}-05 \quad 0.0892$ & $5.76960 \mathrm{E}-05 \quad 0.7623$ \\
\hline Side Upper Leakage & $1.16436 \mathrm{E}-03 \quad 0.1189$ & $3.21004 \mathrm{E}-05 \quad 0.1263$ & $4.65579 \mathrm{E}-05 \quad 0.5542$ \\
\hline Roof Reflection & $7.92388 \mathrm{E}-03 \quad 0.0460$ & $8.25587 \mathrm{E}-05 \quad 0.0870$ & $1.70399 \mathrm{E}-04 \quad 0.3378$ \\
\hline Steel Wall Reflect & $2.42680 \mathrm{E}-050.2353$ & $5.82765 \mathrm{E}-06 \quad 0.2999$ & $3.79737 E-05 \quad 1.0000$ \\
\hline Concrete Floor Effect & $6.02326 \mathrm{E}-03 \quad 0.0452$ & $3.69543 \mathrm{E}-04 \quad 0.0394$ & $1.33840 \mathrm{E}-04 \quad 0.3316$ \\
\hline Total $\mathrm{mrem} / \mathrm{h}$ & $3.47302 \mathrm{E}-02 \quad 0.0216$ & $1.90412 \mathrm{E}-03 \quad 0.0183$ & $6.89650 \mathrm{E}-03 \quad 0.0799$ \\
\hline $3.6 \mathrm{~kg}$ Pu-Container & \multicolumn{2}{|c|}{ csc08anp. out } & csc08ap. out \\
\hline Open End Leakage & 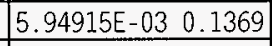 & $1.03303 \mathrm{E}-04 \quad 0.2023$ & $0.00000 E+00 \quad 0.0000$ \\
\hline Front Lower Leakage & $1.68798 \mathrm{E}-02 \quad 0.0942$ & $1.02927 E-03 \quad 0.0646$ & $7.40678 \mathrm{E}-04 \quad 0.4289$ \\
\hline Front Upper Leakage & $7.92102 \mathrm{E}-03 \quad 0.1416$ & $3.75458 \mathrm{E}-04 \quad 0.1009$ & $6.30898 \mathrm{E}-04 \quad 0.3438$ \\
\hline Side Lower Leakage & $6.53228 \mathrm{E}-03 \quad 0.1512$ & $1.18019 \mathrm{E}-04 \quad 0.2268$ & $1.47267 \mathrm{E}-05 \quad 0.9442$ \\
\hline Side Upper Leakage & $2.41222 \mathrm{E}-03 \quad 0.2851$ & $1.91220 \mathrm{E}-05 \quad 0.4054$ & $2.05582 \mathrm{E}-06 \quad 0.8855$ \\
\hline Roof Reflection & $\begin{array}{llll}6.53887 \mathrm{E}-03 & 0.1348 \\
\end{array}$ & $4.83391 \mathrm{E}-05 \quad 0.3522$ & $2.64173 \mathrm{E}-07 \quad 1.0000$ \\
\hline Steel Wall Reflect & $\begin{array}{lll}3.57984 \mathrm{E}-05 & 0.4548\end{array}$ & $2.32480 \mathrm{E}-07 \quad 1.0000$ & $0.00000 E+00 \quad 0.0000$ \\
\hline Concrete Floor Effect & $1.23256 \mathrm{E}-02 \quad 0.0976$ & $5.46129 \mathrm{E}-04 \quad 0.0895$ & $1.91549 \mathrm{E}-05 \quad 0.7516$ \\
\hline Total $\mathrm{mrem} / \mathrm{h}$ & $4.10801 \mathrm{E}-02 \quad 0.0602$ & $1.44262 \mathrm{E}-03 \quad 0.0537$ & $1.38752 \mathrm{E}-03 \quad 0.2774$ \\
\hline
\end{tabular}


HNF-SD-CP-TI-218 Rev. 0

5.8 DOSE RATE ADJACENT TO CALCINER GLOVEBOX SOURCES

\begin{tabular}{|c|c|c|}
\hline Dose Rate Location & Neutron $\mathrm{mrem} / \mathrm{h}$ & Gamma mrem/h \\
\hline $3.5 \mathrm{~kg}$ Pu-Calciner & cca08anp .out & cca08ap. out \\
\hline $\begin{array}{r}x, y, z=-1.68593 \mathrm{E}+02 \\
6.98500 \mathrm{E}+01 \\
1.25971 \mathrm{E}+02\end{array}$ & $7.84649 \mathrm{E}+00 \quad 0.0281$ & $1.25636 \mathrm{E}+00 \quad 0.0689$ \\
\hline $\begin{array}{r}x, y, z=-1.68593 E+02 \\
1.13502 E+02 \\
1.25971 E+02\end{array}$ & $1.63511 \mathrm{E}+00 \quad 0.0170$ & $4.64801 E-02 \quad 0.0533$ \\
\hline $\begin{aligned} x, y, z= & -1.68593 \mathrm{E}+02 \\
& 2.35903 \mathrm{E}+02 \\
& 1.25971 \mathrm{E}+02\end{aligned}$ & $3.44401 E-010.0111$ & $9.66447 \mathrm{E}-03 \quad 0.0706$ \\
\hline $3.5 \mathrm{~kg}$ Pu-Feed Tank & cfto8anp.out & cft08ap.out \\
\hline $\begin{aligned} x, y, z= & 0.00000 E+00 \\
& 3.84534 E+01 \\
& 2.00000 E+02\end{aligned}$ & $6.96388 \mathrm{E}+00 \quad 0.0046$ & $3.09575 \mathrm{E}+02 \quad 0.0041$ \\
\hline $\begin{aligned} x, y, z= & 0.00000 E+00 \\
& 1.13502 E+02 \\
& 2.00000 E+02\end{aligned}$ & $9.86556 \mathrm{E}-010.0060$ & $1.64459 \mathrm{E}-010.0270$ \\
\hline $\begin{aligned} & x, y, z= 0.00000 E+00 \\
& 2.35903 E+02 \\
& 2.00000 E+02 \\
&\end{aligned}$ & $5.13981 \mathrm{E}-020.0248$ & $1.46022 \mathrm{E}-020.1000$ \\
\hline $3.6 \mathrm{~kg} \mathrm{Pu}$-Containers & csc08anp . out & csc08ap.out \\
\hline $\begin{array}{r}x, y, z=-1.26693 \mathrm{E}+02 \\
8.58800 \mathrm{E}+01 \\
9.31068 \mathrm{E}+01 \\
\end{array}$ & $1.24164 \mathrm{E}+010.0428$ & $2.80244 \mathrm{E}-01 \quad 0.0407$ \\
\hline $\begin{aligned} x, y, z= & -1.26693 \mathrm{E}+02 \\
& 1.13502 \mathrm{E}+02 \\
& 9.31068 \mathrm{E}+01\end{aligned}$ & $3.81204 \mathrm{E}+00 \quad 0.0126$ & $9.70591 \mathrm{E}-02 \quad 0.0474$ \\
\hline $\begin{aligned} x, y, z= & -1.26693 \mathrm{E}+02 \\
& 2.35903 \mathrm{E}+02 \\
& 9.31068 \mathrm{E}+01\end{aligned}$ & $4.98551 \mathrm{E}-010.0689$ & $1.14574 \mathrm{E}-02 \quad 0.0564$ \\
\hline
\end{tabular}

\subsection{HANDS AROUND CONTAINERS}

To study the effects of hands in the glovebox. case was modified to form a case by placing a latex. water. latex layers around the radius of the receipt cans. The inside latex is $0.2381 \mathrm{~cm}(0.0937 \mathrm{in.}$ or $3 / 32 \mathrm{in}$.) thick. the water representing hands is $2.54 \mathrm{~cm}(1 \mathrm{in.})$ thick, and the outside latex is $0.2382 \mathrm{~cm}(0.0938 \mathrm{in}$. or $3 / 32 \mathrm{in.})$ thick. The neutron and activation gamma dose rate as well as the direct gamma dose rate is calculated in the water annulus. The containers each hold dry $3.6 \mathrm{~kg}$ Pu or $1.8 \mathrm{~kg} \mathrm{Pu}$.

The tabulated results in Table 5.7. Hand Dose Rate from Receipt Cans, indicates that the dose rate to hands while holding a can is:

- $\quad 680 \mathrm{mrem} / \mathrm{h}$ for a $3.6 \mathrm{~kg}$ Pu can, and

- $475 \mathrm{mrem} / \mathrm{h}$ for a $1.8 \mathrm{~kg} \mathrm{Pu}$ can. 


\begin{tabular}{|c|c|c|}
\hline \multicolumn{3}{|c|}{$\begin{array}{c}\text { Table } 5.7 \text { Hand Dose Rate From Receipt Cans } \\
\text { Source Term used High } 240 \text { Pu Category Plutonium } \\
\text { Neutron. Gamma Activation from mode n.p } \\
\text { Gamma Dose from mode p } \\
3.6 \mathrm{~kg} \mathrm{Pu} \text { can and } 1.8 \mathrm{~kg} \mathrm{Pu} \mathrm{can} \\
\end{array}$} \\
\hline MCNP Results & $\mathrm{mrem} / \mathrm{h}$ & $\pm \sigma$ \\
\hline \multicolumn{3}{|c|}{ crch02np. out, $3.6 \mathrm{~kg}$ Pu can, mode $n, p$} \\
\hline $3.48344 \mathrm{E}+02 \quad 0.0096$ & $3.48344 \mathrm{e}+02$ & 0.0096 \\
\hline $2.60610 E+00 \quad 0.0277$ & $2.60610 \mathrm{e}+00$ & 0.0277 \\
\hline \multicolumn{3}{|c|}{ crch02p.out, $3.6 \mathrm{~kg} \mathrm{Pu}$ can, mode $\mathrm{p}$} \\
\hline $3.29047 E+02 \quad 0.1057$ & $3.29047 \mathrm{e}+02$ & 0.1057 \\
\hline TOTAL mrem $/ \mathrm{h}$ & $6.79997 e+02$ & 0.0562 \\
\hline \multicolumn{3}{|c|}{ crch03np. out, $1.8 \mathrm{~kg}$ Pu can, mode $n, p$} \\
\hline $1.99876 \mathrm{E}+02 \quad 0.0090$ & $1.99876 \mathrm{e}+02$ & 0.0090 \\
\hline $1.39118 E+00 \quad 0.0295$ & $1.39118 \mathrm{e}+00$ & 0.0295 \\
\hline \multicolumn{3}{|c|}{ crch03p.out, $1.8 \mathrm{~kg} \mathrm{Pu}$ can, mode $\mathrm{p}$} \\
\hline $2.75128 \mathrm{E}+02 \quad 0.0971$ & $2.75128 \mathrm{e}+02$ & 0.0971 \\
\hline TOTAL mrem/h & $4.76395 \mathrm{e}+02$ & 0.0599 \\
\hline
\end{tabular}

Calculation of the relative uncertainty for the total dose rate to the hands would be done differently depending upon the assumptions. If the assumption of correlated uncertainties is made, then the uncertainty is:

$(0.0096 * 3.48344 \mathrm{E}+2+0.0277 * 2.60610 \mathrm{E} 0+0.1057 * 3.29047 \mathrm{E}+2) / 6.79997 \mathrm{E}+2$ $=0.0569$ for a combined uncertainty on tota $1 \mathrm{mrem} / \mathrm{h}$.

If the assumption of uncorrelated uncertainties is made, then the uncertainty is:

SQRT $\left((0.0096 * 3.48344 E+2)^{2}+(0.0277 * 2.60610 E 0)^{2}+(0.1057 * 3.29047 E+2)^{2}\right) / 6.79997 E+2$ $=0.0514$ for a combined uncertainty on total $\mathrm{mrem} / \mathrm{h}$.

\subsection{SHIELDS AROUND CONTAINERS}

The reduction of neutron and gamma dose rates from containers is possible by shielding the containers by a neutron shield.

Two geometries were considered:

- cylindrical, and

- Square or box.

Three materials were considered for container neutron shielding material:

- Borated Polyethylene 207.

- Borated Polyethylene 210.

- Lucite or Plexiglas (both are methylmethacrylate polymers). 


\begin{tabular}{|c|c|c|}
\hline \multicolumn{3}{|c|}{$\begin{array}{c}\text { Table } 5.8 \text { Storage Can Cylindrical Shields - Dose Rates } \\
\text { Neutron ( } \mathrm{n} \text {. ) and Photon Dose Rates ( } \mathrm{p} \text {. ( (mrem/h) } \\
\text { Source Term is } 3.6 \mathrm{~kg} \text { Plutonium in Dry PuO, }\end{array}$} \\
\hline $\mathrm{PuO}_{2}+30 \mathrm{~cm}$ & glovebox $+30 \mathrm{~cm}$ & glovebox $+152.8 \mathrm{~cm}$ \\
\hline \multicolumn{3}{|c|}{ csc002an.out, no additional shield } \\
\hline $1.43506 E+010.0695$ & $4.45063 E+00 \quad 0.0462$ & $5.71320 \mathrm{E}-01 \quad 0.0541$ \\
\hline \multicolumn{3}{|c|}{ csc002ap. out, no additional shield } \\
\hline $2.72155 \mathrm{E}-010.1384$ & $1.10414 \mathrm{E}-01 \quad 0.3093$ & $1.19380 \mathrm{E}-02 \quad 0.2616$ \\
\hline \multicolumn{3}{|c|}{ csc003an.out, 1" cylindrical shield of borated poly 207} \\
\hline $8.52330 E+00 \quad 0.0845$ & $2.71223 \mathrm{E}+00 \quad 0.0452$ & $3.60051 \mathrm{E}-01 \quad 0.0616$ \\
\hline \multicolumn{3}{|c|}{ csc003ap.out, 1" cylindrical shield of borated poly 207} \\
\hline $2.21859 \mathrm{E}-010.1557$ & $9.69784 \mathrm{E}-02 \quad 0.3526$ & $1.02630 \mathrm{E}-02 \quad 0.3003$ \\
\hline \multicolumn{3}{|c|}{ csc003bn.out, 1" cylindrical shield of borated poly 210} \\
\hline $8.24647 \mathrm{E}+00 \quad 0.0486$ & $2.84517 \mathrm{E}+00 \quad 0.0462$ & $3.69602 \mathrm{E}-01 \quad 0.0549$ \\
\hline \multicolumn{3}{|c|}{ csc003bp.out, 1" cylindrical shield of borated poly 210} \\
\hline $2.30997 E-010.1517$ & $9.78125 E-02 \quad 0.3456$ & $1.03615 \mathrm{E}-02 \quad 0.2933$ \\
\hline \multicolumn{3}{|c|}{ csc003en out, 1" cylindrical shield of Lucite } \\
\hline $8.88006 \mathrm{E}+00 \quad 0.0505$ & $3.03598 E+00 \quad 0.0459$ & $3.97229 E-01 \quad 0.0479$ \\
\hline \multicolumn{3}{|c|}{ csc003ep.out, 1" cylindrical shield of Lucite } \\
\hline $2.28606 \mathrm{E}-01 \quad 0.1533$ & $9.69384 \mathrm{E}-02 \quad 0.3503$ & $1.01284 \mathrm{E}-02 \quad 0.3009$ \\
\hline \multicolumn{3}{|c|}{$\begin{array}{c}\text { Table } 5.9 \text { Storage Can Square Shields - Dose Rates } \\
\text { Neutron ( } \mathrm{n} \text {. ) and Photon Dose Rates ( p. ) (mrem/h) } \\
\text { Source Term is } 3.6 \mathrm{~kg} \text { Plutonium in Dry Pu0, }\end{array}$} \\
\hline $\mathrm{PuO}_{2}+30 \mathrm{~cm}$ & glovebox $+30 \mathrm{~cm}$ & glovebox $+152.8 \mathrm{~cm}$ \\
\hline \multicolumn{3}{|c|}{ csc002an. out, no additional shield } \\
\hline $1.43506 \mathrm{E}+01 \quad 0.0695$ & $4.45063 E+00 \quad 0.0462$ & $5.71320 E-010.0541$ \\
\hline \multicolumn{3}{|c|}{ csc002ap.out, no additional shield } \\
\hline $2.72155 \mathrm{E}-01 \quad 0.1384$ & $1.10414 \mathrm{E}-01 \quad 0.3093$ & $1.19380 \mathrm{E}-02 \quad 0.2616$ \\
\hline \multicolumn{3}{|c|}{ csc003cn.out, 1" square shield of borated poly 207} \\
\hline $1.44633 \mathrm{E}+010.3689$ & $2.89555 E+00 \quad 0.0601$ & $3.80961 E-01 \quad 0.0565$ \\
\hline \multicolumn{3}{|c|}{ csc003cp.out, 1" square shield of borated poly 207} \\
\hline $2.14533 \mathrm{E}-010.1526$ & $9.24376 \mathrm{E}-02 \quad 0.3626$ & $9.88685 \mathrm{E}-03 \quad 0.3047$ \\
\hline \multicolumn{3}{|c|}{ csc003dn.out, 1" square shield of borated poly 210} \\
\hline $8.41078 E+00 \quad 0.0525$ & $2.82536 \mathrm{E}+00 \quad 0.0437$ & $3.81597 \mathrm{E}-01 \quad 0.0644$ \\
\hline \multicolumn{3}{|c|}{ csc003dp.out, 1" square shield of borated poly 210} \\
\hline $2.22954 \mathrm{E}-01 \quad 0.1508$ & $9.62518 \mathrm{E}-02 \quad 0.3510$ & $1.03783 \mathrm{E}-02 \quad 0.2939$ \\
\hline \multicolumn{3}{|c|}{ csc003fn. out, 1" square shield of Lucite ${ }^{\Phi}$} \\
\hline $1.05240 E+010.0664$ & $3.31358 \mathrm{E}+00 \quad 0.0492$ & $4.14267 \mathrm{E}-010.0551$ \\
\hline \multicolumn{3}{|c|}{ csc003fp.out, 1" square shield of Lucite } \\
\hline $2.21675 \mathrm{E}-010.1508$ & $9.51206 \mathrm{E}-02 \quad 0.3542$ & \\
\hline
\end{tabular}


HNF-SD-CP-TI-218 ReV. 0

\subsection{REFERENCES}

Aftanas, B. L., April 30, 1996, PFP Solution Stabi7ization, WHC-SD-CP-FDC-003 Rev. 0, Westinghouse Hanford Company, Richland, Washington.

ANSI/ANS-6.1.1-1977, 1977, Neutron and Gamma-Ray Flux-to-Dose-Rate Factors. American Nuclear Society, La Grange Park, I1linois

ANSI/ANS-6.1.1-1991. 1992. Neutron and Gamma-Ray Fluence-to-Dose Factors, American Nuclear Society, La Grange Park. I1linois

Briesmeister, J. F., Editor, November 1993, MCNP - A General Monte Carlo N-Particle Transport Code, Version 4A, LA-12625, Los Alamos National Laboratory, Los Alamos, New Mexico.

Carter, L. L., June 10, 1996, Certification of MCNP Version $4 A$ for the WHC Computer Platforms, ECN-630773, WHC-SD-MP-SWD-30001, Rev. 8, Westinghouse Hanford Company, Richland, Washington.

Carter, R. D., G. R. Kiel and K. R. Ridgeway, 1968, Criticality Handbook. ARH-600, Atlantic Richfield Hanford Company, Richland, Washington.

Compton, J. A., January 23, 1997, Prototype Vertical Calciner Introductory Guide, HNF-SD-CP-LB-037, Rev. 0. Babcock \& Wilcox Hanford Company, Richland, Washington.

Croff, A. G. July 1980. A User's Manual for the ORIGEN2 Computer Code, ORNL/TM-7175, Oak Ridge National Laboratory, Oak Ridge, Tennessee.

Durni1, J. F. and S. B. Dutta, January 26, 1996, Location Assessment of Vertical Calciner, WHC-SD-CP-TI-200, Rev. 0, Westinghouse Hanford Company, Richland, Washington.

Geiger, J. L., 1995a, CSER 95-005: PFP Vertica7 Denitration Ca7ciner, WHC-SD-SQA-20392, Rev. 0, Westinghouse Hanford Company, Richland, Washington.

Geiger. J. L., 1995b. CSER 95-005 Addendum I Stainless Steel Insulation, WHC-SD-SQA-CSA-20404, Rev. 0, Westinghouse Hanford Company, Richland. Washington.

General Electric. February 10, 1993, 234-5Z Building Elevation of Concrete Wal7s Enclosing Process Area, H-2-16174, General Electric Co. Hanford Works, Richland, Washington.

Hillesland. K. E., May 1997, CSER 97-004: PFP Production Denitration Calciner System, HNF-SD-SQA-CSA-529, Rev. 0. Fluor Daniel Northwest, Richland, Washington.

Jaeger, R. G., E. P. Blizard, A. B. Chilton, M. Grotenhuis, A. Honig, Th. A. Jaeger, H. H. Eisenlohr, Editors, 1975, Engineering Compendium on Radiation Shielding, Volume II Shielding Materials. Springer-Verlag. New York. New York. 
HNF-SD-CP-TI-218 Rev. 0

Merrick, S. B., and P. M. Rickords, January 29, 1996, Technical Assessment of the PFP Vertical Direct Denitration Calciner. WHC-SD-CP-TA-009, Rev. 0, Westinghouse Hanford Company, Richland. Washington.

Schmittroth, F. A., September 1993, Conversion of ORIGEN2 to Sun Workstations, ECN-186709, WHC-SD-NR-SWD-006, Rev. 0-A, Westinghouse Hanford Company, Richland. Washington.

Stubbs, A. M., 1997, Plutonium Finishing Plant Process Flow Document: Calcination of Plutonium Nitrate Solution. PFD-Z-300-001. Rev. A-0. Babcock and Wilcox Hanford Company, Richland, Washington.

U. S. DOE, July 7, 1994, First Floor Plan Part 'C' 234-5Z Building, H-2-16170, Rev. 17, U.S. Department of Energy, DOE Field Office, Richland, Westinghouse Hanford Company, Richland, Washington.

U. S. DOE, Expected in 1997, Calciner Assembly, H-2-95609, Rev. 0 U.S. Department of Energy, Richland Operations Office, B \& W Hanford Company, Richland. Washington.

Wittekind, W. D., July 19, 1994, Software Configuration Control Package for the ORIGEN2 Code. WHC-SD-NR-SWD-006, Westinghouse Hanford Company, Richland. Washington. 
HNF-SD-CP-TI-218 Rev. 0

APPENDIX A

INDEPENDENT REVIEW COMMENTS AND CHECKLIST 
HNF-SD-CP-TI-218 Rev. 0

This page intentionally left blank 
HNF-SD-CP-TI-218 Rev. 0

\section{TECHNICAL PEER REVIEW}

H.J. Goldberg of the Criticality and Shielding group in Specialty Engineering carried out an independent, technical peer review of this shielding calculation. 
HNF-SD-CP-TI-218 Rev. 0

CHECKLIST FOR INDEPENDENT TECHNICAL REVIEW

NUMBER: $\quad$ HNF-SD-CP-TI-218 Rev. 0

DOCUMENT REVIEWED: PFP Vertical Calciner Shield Wall Dose Rate Calculations Using MCNP

AUTHOR(S): _warren D. Wittekind

I. Method(s) of Review

( $)$ Input data checked for accuracy

( ) Independent calculation performed

() Hand calculation

( ) Alternate computer code:

( ) Comparison to experiment or previous resuits

( ) Alternate method (define)

II. Check]ist (either check or enter NA if not applied)

( T) Task completely defined

( 2 Activity consistent with task specification

( $)$ Necessary assumptions explicitly stated and supported

( 1 Resources properly identified and referenced

(1.) Resource documentation appropriate for this application

(1) Input data explicitly stated

( $)$ Input data verified to be consistent with original source

(r) Geometric model adequate representation of actual geometry

(f) Material properties appropriate and reasonable

WAs Mathematical derivations checked including dimensional consistency

(W/A) Hand calculations checked for errors

(1) Assumptions explicitly stated and justified

(a) Computer software appropriate for task and used within range of validity

NA) Use of resource outside range of established validity is justified

(1) Software runstreams correct and consistent with results

(1) Software output consistent with input

WA Results consistent with applicable previous experimental or analytical findings

( $)$ Results and conclusions address all points and are consistent with

task requirements and/or established limits or criteria

(N) Conclusions consistent with analytical results and established Timits

(V) Uncertainty assessment appropriate and reasonable

( ) Other (define)

III. Comments:

The variances on the results are relative errors (in \%) and not or. This shuld be made explicit.

IV

REVIEWER :

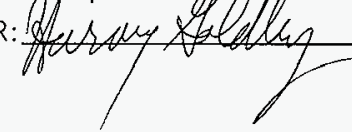

DATE

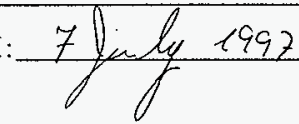


HNF-SD-CP-TI-218 Rev. 0

APPENDIX B

ORIGEN2 PLUTONIUM INPUT FILE AND OUTPUT RESULTS 
HNF-SD-CP-TI-218 Rev. 0

This page intentionally left blank 
HNF-SD-CP-TI-218 Rev. 0

ORIGEN2 PLUTONIUM INPUT FILE

$-1$

$-1$

TIT ORIGEN-II CALCULATIONS FOR CALCINER PU02

LIP $\quad 0000$

LIB $\quad 012320120220393010$

PHO 10110210310

RDA 1 gram

INP $\quad-1 \quad 1 \quad-1 \quad-1 \quad 1 \quad 1$

MOV $\quad-1 \quad 1 \quad 0 \quad 0.008908685969$ original vector low Pu-240 wt\%

INP $\quad-2 \quad 1 \quad-1 \quad-1 \quad 1 \quad 1$

MOV $\quad-2 \quad 5 \quad 0 \quad 0.008745080892$ original vector mid Pu-240 wt\%

INP $\quad-3 \quad 1 \quad-1 \quad-1 \quad 1 \quad 1$

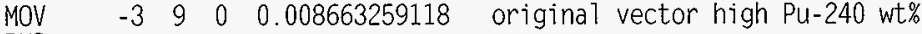

BUP

DEC $\quad 1.0$

DEC $\quad 5.0$

DEC $\quad 10.0$

DEC $\quad 1.0$

DEC $\quad 5.0$

DEC $\quad 10.0$

DEC $\quad 1.0$

DEC $\quad 5.0$

DEC $\quad 10.0$

BUP

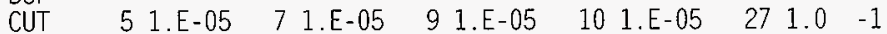

RDA ACTIVATION PRODUCTS OUTPUT TABLE OPTIONS

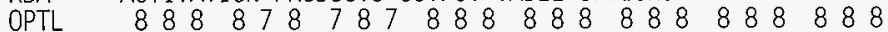

RDA FISSION PRODUCTS OUTPUT TABLE OPTIONS

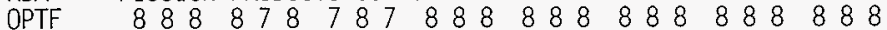

RDA ACTINIDE AND DAUGHTER PRODUCTS OUTPUT TABLE OPTIONS

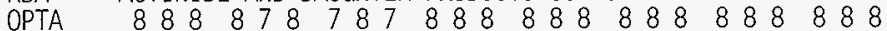

HED $\quad 1$ TO 00 D

HED 2 TO 1 D

HED $\quad 310 \quad 50$

HED $\quad 41010 \mathrm{D}$

HED $\quad 5 \mathrm{md} \quad 0 \mathrm{D}$

HED $\quad 6 \mathrm{md} 1 \mathrm{D}$

HED $\quad 8 \mathrm{md} 10 \mathrm{D}$

HED $\quad 9$ hi $\quad 0$ D

HED $\quad 10$ hi 1 D

HED 11 hi 5 D

HED 12 hi $10 \mathrm{D}$

OUT $121-1 \quad 0$

STP 4

$\begin{array}{rrrrrrrrr}2 & 942380 & 0.01 & 942390 & 92.40 & 942400 & 6.12 & 942410 & 0.26 \\ 2 & 942420 & 0.04 & 952410 & 0.18 & 000000 & 0.00 & 000000 & 0.00 \\ 1 & 80160 & 13.25 & 000000 & 0.00 & 000000 & 0.00 & 000000 & 0.00 \\ 0 & & & & & & & & \\ 2 & 942380 & 0.15 & 942390 & 85.63 & 942400 & 12.45 & 942410 & 1.20 \\ 2 & 942420 & 0.37 & 952410 & 1.06 & 000000 & 0.00 & 000000 & 0.00 \\ 1 & 80160 & 13.49 & 000000 & 0.00 & 000000 & 0.00 & 000000 & 0.00 \\ 0 & & & & & & & & \end{array}$ 


\section{HNF-SD-CP-TI-218 Rev. 0}

$\begin{array}{lrrrrrrrr}2 & 942380 & 0.18 & 942390 & 80.06 & 942400 & 17.84 & 942410 & 1.22 \\ 2 & 942420 & 0.73 & 952410 & 1.79 & 000000 & 0.00 & 000000 & 0.00 \\ 1 & 80160 & 13.61 & 000000 & 0.00 & 000000 & 0.00 & 000000 & 0.00 \\ 0 & & & & & & & & \\ \text { END } & & & & & & & & \end{array}$




\section{ORIGEN2 PLUTONIUM OUTPUT RESULTS}

5 SUMMARY TABLE: CONCENTRATIONS, GRAMS
1000
lo 10
lo 50
$10.0 \mathrm{D}$ ma $10 \mathrm{D}$
mo $0 \quad 0$
md $1 \mathrm{D}$
mod $5 \mathrm{D}$
hi $10 \mathrm{D}$
hi OD
hi $1 \mathrm{D}$ hi $5 \mathrm{D}$

PU238

PU239

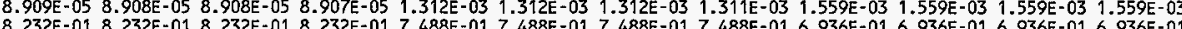

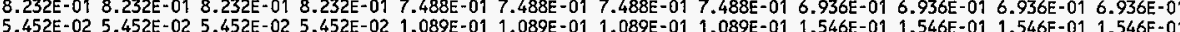

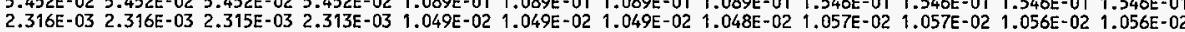
3.563E-04 3.563E-04 3.563E-04 3.563E-04 3.236E-03 3.236E-03 3.236E-03 3.236E-03 6.324E-03 6.324E-03 6.324E-03 6.324E-03 1.604E-03 1.604E-03 1.605E-03 1.607E-03 9.270E-03 9.271E-03 9.276E-03 9.283E-03 $1.551 E-02 \quad 1.551 E-02 \quad 1.551 E-02 \quad 1.552 E-02$

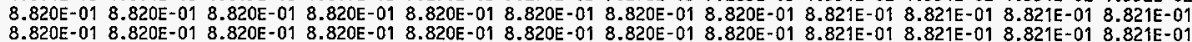

\section{SUMMARY TABLE: RADIOACTIVITY, CURIES}
1000
1010 lo $5 \mathrm{D}$
10.0D md 10
md 00
nd 10
md $5 \mathrm{D}$
hi $10 \mathrm{D}$
hi OD
hi 1 D
hi 50

U237

PU238

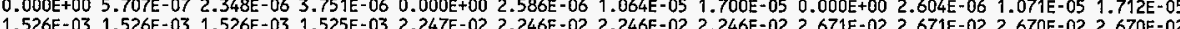

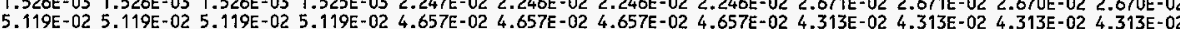
1.243E-02 1.243E-02 1.243E-02 1.243E-02 2.482E-02 2.482E-02 2.482E-02 2.482E-02 3 .523E-02 3.523E-02 3.523E-02 3.523E-02 2.387E-01 2.387E-01 2.386E-01 2.384E-01 1.082E+00 1.081E+00 1.081E+00 1.080E+00 1.089E+00 1.089E+00 1.089E+00 1.088E+00

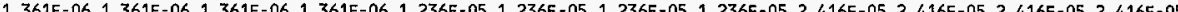
$5.506 \mathrm{E}-035.507 \mathrm{E}-035 \mathrm{5} 11 \mathrm{E}-035.516 \mathrm{E}-033.183 \mathrm{E}-023.183 \mathrm{E}-023.185 \mathrm{E}-023 \mathrm{3}$ $3.094 \mathrm{E} 013.093 \mathrm{E}-013.092 \mathrm{E}-013.091 \mathrm{E}-0113 \mathrm{~B}$

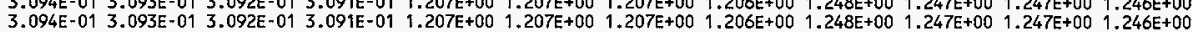


BASIS=

ORIGEN-II CALCULATIONS FOR CALCINER PUOZ (ALPHA, N) NEUTRON SOURCE, NEUTRONS/SEC
Lo 00 lo 10
lo $5 \mathrm{D}$
10.0D ma $10 \mathrm{D}$
md 0 D
md $1 \mathrm{D}$
md $5 \mathrm{D}$ hi $10 \mathrm{D}$
hi OD
hi $1 \mathrm{D}$ hi $5 \mathrm{D}$

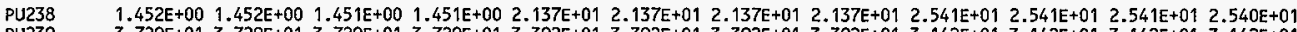
PU239 3.729E+01 3.729E $\$ 013.729 E+013.729 E+013.392 E+013.392 E+013.392 E+013.392 E+01 \quad 3.142 E+013.142 E+01 \quad 3.142 E+013.142 E+01$ PU240 $9.417 E+009.417 E+00 \quad 9.417 E+00 \quad 9.417 E+00 \quad 1.881 E+01 \quad 1.881 E+01 \quad 1.881 E+01 \quad 1.881 E+012.670 E+012.670 E+012.670 E+012.670 E+01$ AN241 5.281E+00 5.282E+00 5.286E+00 5.291E+00 3.053E+01 3.053E+01 3.055E+01 3.057E+01 $5.107 E+01 \quad 5.107 E+01 \quad 5.109 E+01 \quad 5.111 E+01$

TOTALS

TABLE

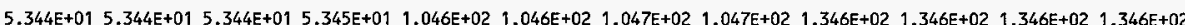

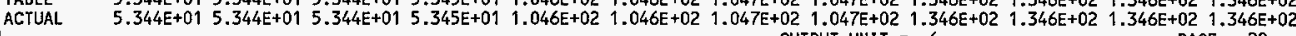
$1^{\text {ACTUA }}$ ORIGEN-II CALCULATIONS FOR CALCINER PUO2 SPONTANEOUS FISSION NEUTRON SOURCE, NEUTRONS/SEC BASIS= OUTPUT UNIT $=6$

PAGE 28
LO 00 lo 10 LO 50
10.00 md 100
md $O D$ and 10
md $5 \mathrm{D}$
hi $10 \mathrm{D}$
hi 0 D
hi $1 \mathrm{D}$ hi $5 \mathrm{D}$

PU238 2.367E-01 2.367E-01 2.367E-01 2.367E-01 $3.486 E+00 \quad 3.486 E+00 \quad 3.485 E+00 \quad 3.485 E+00 \quad 4.144 E+00 \quad 4.144 E+00 \quad 4.143 E+00 \quad 4.143 E+00$ PU240 4.964E+01 4.964E+01 $4.964 E+01 \quad 4.964 E+01 \quad 9.913 E+01 \quad 9.913 E+01 \quad 9.913 E+019.913 E+01 \quad 1.407 E+02 \quad 1.407 E+02 \quad 1.407 E+02 \quad 1.407 E+02$ PU242 6.007E-01 6.007E-01 6.007E-01 6.007E-01 5.454E+00 5.454E+00 5.454E+00 5.454E+00 1.066E+011.066E+011.066E+01 $1.066 E+01$

\section{0.......}

TOTALS

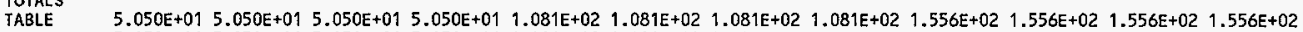
ACTUAL $\quad 5.050 E+01 \quad 5.050 E+01 \quad 5.050 E+01 \quad 5.050 E+01 \quad 1.081 E+02 \quad 1.081 E+02 \quad 1.081 E+02 \quad 1.081 E+02 \quad 1.556 E+02 \quad 1.556 E+02 \quad 1.556 E+02 \quad 1.556 E+02$ 0-.......

OVERALL

TOTALS

TABLE

ACTUAL

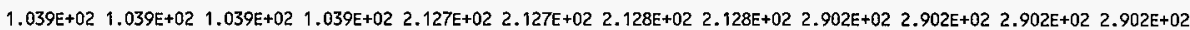

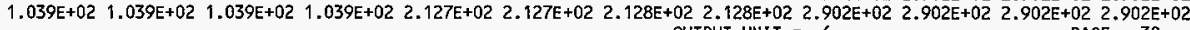


EMEAN

ORIGEN-I I CALCULATIONS FOR CALCINER PUO2

POWER $=1.00 \mathrm{MH}$, BURNUP $=$ i. MWD, FLUX $=1.00 \mathrm{~N}+00 \mathrm{~N} / \mathrm{CM} \star * 2-\mathrm{SEC}$

18 GROUP PHOTON RELEASE RATES, PHOTONS/SECOND

BASIS=

$\begin{array}{lllllllllllllll}1.500 E-02 & 2.343 E+08 & 2.343 E+08 & 2.344 E+08 & 2.345 E+08 & 6.420 E+08 & 6.421 E+08 & 6.425 E+08 & 6.428 E+08 & 8.923 E+08 & 8.924 E+08 & 8.928 E+08 & 8.932 E+08\end{array}$

$2.500 E-02 \quad 5.152 E+06 \quad 5.154 E+06 \quad 5.160 E+06 \quad 5.166 E+06 \quad 2.978 E+07 \quad 2.979 E+07 \quad 2.982 E+07 \quad 2.985 E+07 \quad 4.983 E+07 \quad 4.983 E+07 \quad 4.986 E+07 \quad 4.989 E+07$

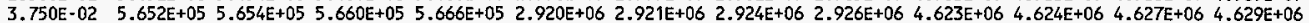

$5.750 \mathrm{E}-02 \quad 7.632 \mathrm{E}+07 \quad 7.634 \mathrm{E}+07 \quad 7.643 \mathrm{E}+07 \quad 7.652 \mathrm{E}+07 \quad 4.386 \mathrm{E}+08 \quad 4.387 \mathrm{E}+08 \quad 4.391 \mathrm{E}+08 \quad 4.395 \mathrm{E}+08 \quad 7.334 \mathrm{E}+08 \quad 7.335 \mathrm{E}+087.339 \mathrm{E}+08 \quad 7.343 \mathrm{E}+08$

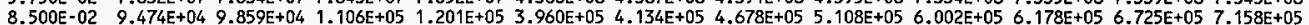

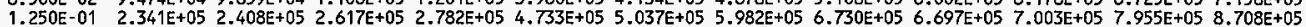

$2.250 \mathrm{E}-01 \quad 2.741 \mathrm{E}+04 \quad 3.246 \mathrm{E}+04 \quad 4.817 \mathrm{E}+04 \quad 6.058 \mathrm{E}+04 \quad 4.504 \mathrm{E}+04 \quad 6.790 \mathrm{E}+04 \quad 1.391 \mathrm{E}+05 \quad 1.953 \mathrm{E}+05 \quad 5.630 \mathrm{E}+04 \quad 7.932 \mathrm{E}+04 \quad 1.510 \mathrm{E}+05 \quad 2.076 \mathrm{E}+05$

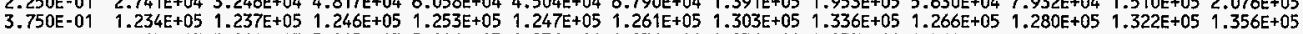

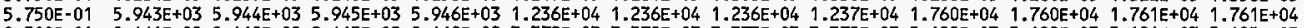

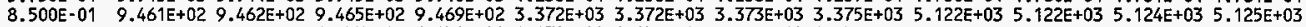

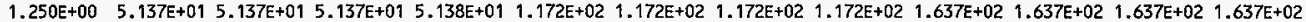

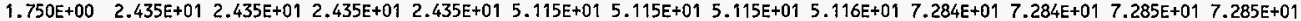

$\begin{array}{lllllllllllllll}2.250 \mathrm{E}+00 & 1.393 \mathrm{E}+01 & 1.393 \mathrm{E}+01 & 1.393 \mathrm{E}+01 & 1.393 \mathrm{E}+01 & 2.934 \mathrm{E}+01 & 2.934 \mathrm{E}+01 & 2.934 \mathrm{E}+01 & 2.934 \mathrm{E}+01 & 4.183 \mathrm{E}+01 & 4.183 \mathrm{E}+01 & 4.183 \mathrm{E}+01 & 4.183 \mathrm{E}+01\end{array}$

$2.750 \mathrm{E}+007.995 \mathrm{E}+007.995 \mathrm{E}+007.995 \mathrm{E}+007.995 \mathrm{E}+001.687 \mathrm{E}+011.687 \mathrm{E}+011.687 \mathrm{E}+011.687 \mathrm{E}+012.408 \mathrm{E}+012.408 \mathrm{E}+012.408 \mathrm{E}+012.408 \mathrm{E}+01$

$3.500 \mathrm{E}+007.064 \mathrm{E}+007.064 \mathrm{E}+007.064 \mathrm{E}$

2.136E+01 $2.136 \mathrm{E}+01$

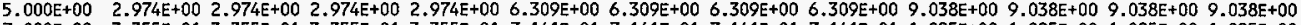

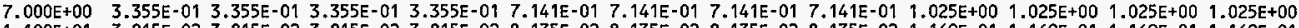

$1.100 \mathrm{E}+01 \quad 3.815 \mathrm{E}-02 \quad 3.815 \mathrm{E}-02 \quad 3.815 \mathrm{E}-02$ 3.815E-02 8.135E-02 $8.135 \mathrm{E}-02$ 8.135E-02 8.135E-02 $1.169 \mathrm{E}-011.169 \mathrm{E}-01 \quad 1.169 \mathrm{E}-011.169 \mathrm{E}-01$

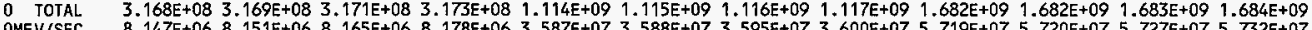

OMEV/SEC $\quad 8.147 E+06 \quad 8.151 E+06 \quad 8.165 E+06 \quad 8.178 E+06 \quad 3.587 E+07 \quad 3.588 E+07 \quad 3.595 E+07 \quad 3.600 E+07 \quad 5.719 E+07 \quad 5.720 E+07 \quad 5.727 E+07 \quad 5.732 E+07$ 18 GROUP SPECIFIC ENERGY RELEASE RATES, MEV/WATT-SEC BASIS=

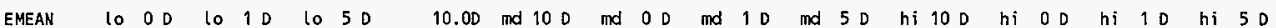

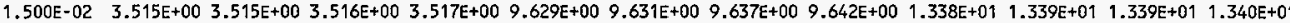
2.500E-02 1.288E-01 1.289E-01 1.290E-01 1.292E-01 7.446E-01 7.448E-01 7.455E-01 7.462E-01 $1.246 \mathrm{E}+00 \quad 1.246 \mathrm{E}+00 \quad 1.247 \mathrm{E}+00 \quad 1.247 \mathrm{E}+00$ 3.750E-02 2.120E-02 2.120E-02 2.123E-02 2.125E-02 1.095E-01 1.095E-01 1.096E-01 1.097E-01 1.734E-01 1.734E-01 1.735E-01 1.736E-01 $\begin{array}{lllllllllllllllll}5.750 \mathrm{E}-02 & 4.388 \mathrm{E}+00 & 4.390 \mathrm{E}+00 & 4.394 \mathrm{E}+00 & 4.400 \mathrm{E}+00 & 2.522 \mathrm{E}+01 & 2.523 \mathrm{E}+01 & 2.525 \mathrm{E}+01 & 2.527 \mathrm{E}+01 & 4.217 \mathrm{E}+01 & 4.218 \mathrm{E}+01 & 4.220 \mathrm{E}+01 & 4.222 \mathrm{E}+01\end{array}$

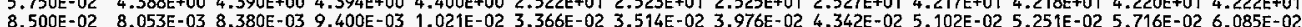

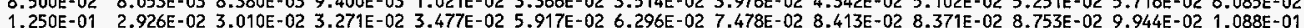

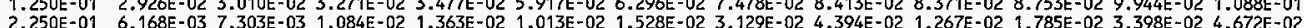

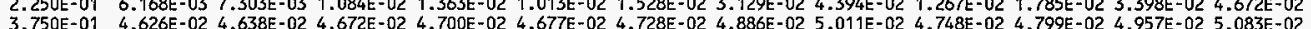

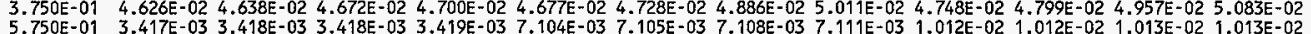

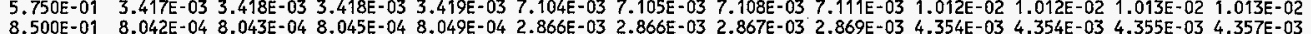

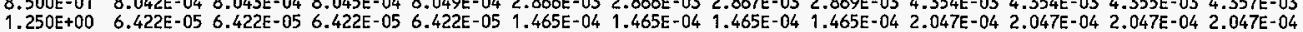

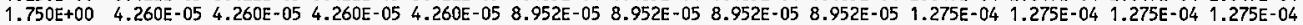
2.250E+00 3.134E-05 3.134E-05 3.134E-05 3.134E-05 6.601E-05 6.601E-05 6.601E-05 6.601E-05 9.412E-05 9.412E-05 9.412E-05 9.412E-05

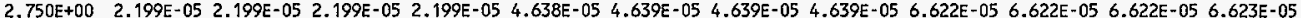
$3.500 E+00 \quad 2.472 E-052.472 E-052.472 E-05$ 2.472E-05 5.228E-05 5.228E-05 5.228E-05 5.228E-05 7.475E-05 7.475E-05 $7.475 E-057.475 E-05$

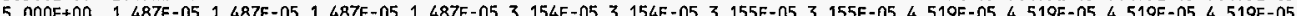
(1) $7.000 \mathrm{E}+00 \quad 2.349 \mathrm{E}-06 \quad 2.349 \mathrm{E}-06$ 2.349E-06 2.349E-06 4.999E-06 4.999E-06 4.999E-06 4.999E-06 7.176E-06 7.176E-06 7.176E-06 7.176E-06

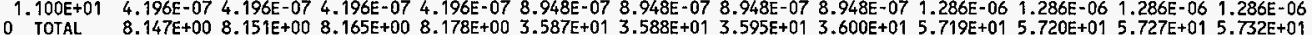

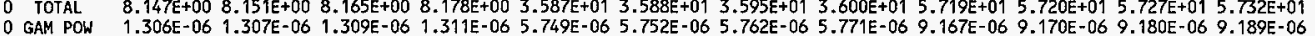


HNF-SD-CP-TI-218 Rev. 0

This page intentionally left blank 
HNF-SD-CP-TI-218 Rev. 0

APPENDIX C

NEUTRON SHIELDED OPERATOR DOSE RATE MCNP INPUT FILES

CCA08ANP. INP Vertical Calciner, $3.5 \mathrm{~kg} \mathrm{Pu}$, neutron and capture gammas

CCA08AP. INP Vertical Calciner, $3.5 \mathrm{~kg}$ Pu, gamma ray calculation

CFT08ANP. INP Feed Tank, $3.5 \mathrm{~kg}$ Pu, neutron and capture gammas

CFT08AP. INP Feed Tank. $3.5 \mathrm{~kg}$ Pu, gamma ray calculation

CSC08ANP. INP Storage Can. $3.6 \mathrm{~kg} \mathrm{Pu}$, neutron and capture gammas

CSCO8AP. INP Storage Can, $3.6 \mathrm{~kg} \mathrm{Pu}$, gamma ray calculation 
HNF-SD-CP-TI-218 Rev. 0

This page intentionally left blank 


\section{NEUTRON SHIELDED OPERATOR DOSE RATE MCNP INPUT FILES}

CCA08ANP.INP Vertical Calciner, $3.5 \mathrm{~kg} \mathrm{Pu}$, neutron \& capture gammas

NEUTRON Dose Rate Calcs for calciner CCAOBANP

c CALCINER PUO2 Neutron Source is in Cell 3

$N$ Shield Lucite - $1.185 \mathrm{~g} / \mathrm{cm}^{3}-4$ inches top, 5 inches bottom

Lower Shields are 1/8 inch of Stainless Steel (both sides)

Upper shields are $3 / 4$ inch of Glass (no Boron)(both sides)

Full Room Model with Concrete Walls, Roof, and Metal Partitions $x x-x x$ yy $-y y \quad z z-z z$ rr $-r r \$$

$110-1.29 \mathrm{e}-3$

$\begin{array}{lll}2 & 7 & -7.84 \\ 3 & 4 & -5.00\end{array}$

$\begin{array}{lcl}3 & 4 & -5.00 \\ 4 & 10 & -1.29 e-3\end{array}$

$\begin{array}{llll}5 & 7 & -7.84\end{array}$

$\begin{array}{lcc}6 & 10 & -1.29 e-3 \\ 7 & 7 & -7.84\end{array}$

$\begin{array}{lcc}8 & 15 & -0.387\end{array}$

$9 \begin{array}{lll}9 & 10 & -1.29 \mathrm{e}-3\end{array}$

$107-7.84$

$\begin{array}{lll}11 & 6 & -7.84 \\ 11 & -0.793\end{array}$

$1210-1.29 e-3$

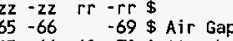

$\begin{array}{lllll}65 & -66 & 69 & -70 & \$ \text { Ss tube inside }\end{array}$

\begin{tabular}{lllll}
65 & -66 & 70 & -71 & $\$$ \\
\hline & PuO2 product
\end{tabular}

$\begin{array}{llll}66 & -67 & -75 & \$ \text { SS dome inside }\end{array}$

$\begin{array}{lllll}66 & -67 & 75 & -76 & \$ \text { SS dome }\end{array}$

$\begin{array}{llll}66 & -67 & 76 & -71 \\ \text { \$ Air Annulus }\end{array}$

$\begin{array}{lllll}65 & -67 & 71 & -72 & \$ \text { S }\end{array}$ S tube outside

$\begin{array}{llll}65 & -67 & 72 & -74\end{array}$ L Lower Insulation

$\begin{array}{lll}67 & -68 & -71 \text { \$ Air Gap }\end{array}$

$\begin{array}{lllll}67 & -68 & 71 & -72 & \$ \text { S }\end{array}$ S tube outside

$\begin{array}{lllll}67 & -68 & 72 & -73 & \$\end{array}$ Upper Insulation

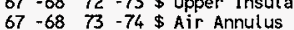

$1310-1.290-3 \quad 24-25 \quad 34-359-15 \quad(-65: 68: 74)$ \$ Inside Glovebox

$14 \quad 7-7.84 \quad 23-26 \quad 33-36 \quad 8-16(-24: 25:-34: 35:-9: 15)$ \$ Ss Inside

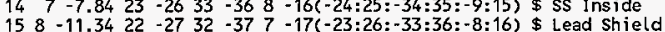

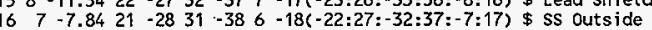

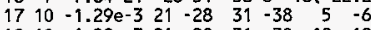

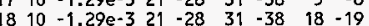

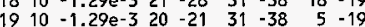

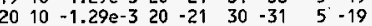

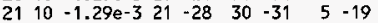

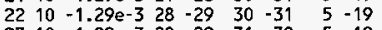

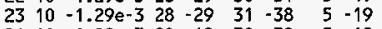

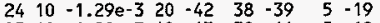

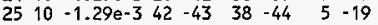

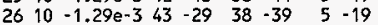

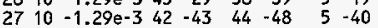

$\begin{array}{lllllllll}28 & 7 & -7.84 & 55 & -43 & 48 & -49 & 5 & -40\end{array}$

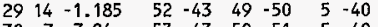

$\begin{array}{lllllllll}30 & 7 & -7.84 & 53 & -43 & 50 & -51 & 5 & -40 \\ 31 & 10 & -1.29 e-3 & 56 & -43 & 51 & -47 & 5 & -40\end{array}$

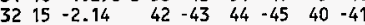

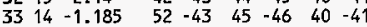

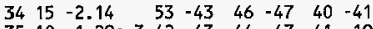

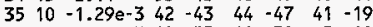

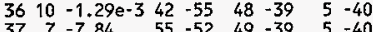

$\begin{array}{lllllll}55 & -52 & 49 & -39 & 5 & -40\end{array}$

$\begin{array}{lllllllll}38 & 14 & -1.185 & 52 & -53 & 50 & -39 & 5 & -40 \\ 39 & 7 & -7.84 & 53 & -56 & 51 & -39 & 5 & -40\end{array}$

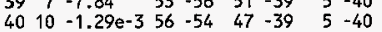

$\begin{array}{lllllllrl}40 & 10 & -1.29 e-3 & 56 & -54 & 47 & -39 & 5 & -40 \\ 41 & 15 & -2.14 & 42 & -52 & 45 & -39 & 40 & -41\end{array}$

$\begin{array}{lllllllll}42 & 14 & -1.185 & 52 & -53 & 46 & -39 & 40 & -4\end{array}$

$\begin{array}{lllllllll}43 & 15 & -2.14 & 53 & -54 & 47 & -39 & 40 & -41\end{array}$

$\$$ Top L Middle $1.0^{\prime \prime}$

$\$$ Top L Inside $3 / 4^{\prime \prime}$

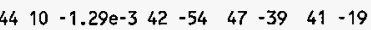

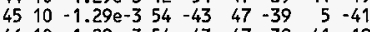

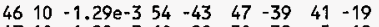

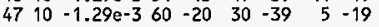
$4810-1.29 e-3 \quad 60-20 \quad 63-30 \quad 5-19$

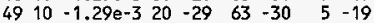

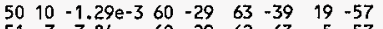
$\begin{array}{lllllllll}51 & 7 & -7.84 & 60 & -29 & 62 & -63 & 5 & -57\end{array}$ $\begin{array}{lllllllll}52 & 7 & -7.84 & 60 & -29 & 39 & -64 & 5 & -57\end{array}$ $\begin{array}{lllllllll}53 & 11 & -2.30 & 59 & -60 & 62 & -64 & 5 & -57\end{array}$

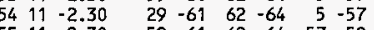
$\begin{array}{lllllllll}55 & 11 & -2.30 & 59 & -61 & 62 & -64 & 57 & -58 \\ 56 & 11 & -2.30 & 59 & -61 & 62 & -64 & 4 & -5\end{array}$

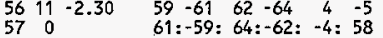

Above Shield Behind $n$ Shield Behind $n$ Shield $\$$ Roon, Left Side $\$$ Room, Left Back $\$$ Room, Back Part $\$$ Roon to Ceiling $\$$ Ss Wall Back $\$$ Ss Wall Back $\$$ Concrete Wall Left \$ Concrete Wall Right $\$$ Concrete Roof

$\$$ Concrete Floor

$\$$ OUTSIDE WORLD
$\$$ FEED TANK O.R. - (5/16"-0.040") $\$$ FEED TANK O.R.

$\$$ FEED TANK FLANGE $0 . R$.

$\$$ CONCRETE FLOOR bottom (24" thick)

$\$$ CONCRETE FLOOR surface

$\$$ GLOVEBOX bottom surface

\$ GLOVEBOX Stainless steel (3/32")

$\$$ GLOVEBOX Lead Shield (3/16")

\$ GLOVEBOX stainless steel (3/32")

$\$$ FEED TANK Bottom $F$ lange bottom

$\$$ FEED TANK Bottom Flange top

$\$$ FEED TANK Solution level $(10 \mathrm{~L})$

$\$$ FEED TANK TOP Flange bottom

$\$$ FEED TANK TOP Flange top

\$ GLOVEBOX top inside surface

\$ GLOVEBOX stainless steel (3/32")

$\$$ GLOVEBOX Lead Shield $\left(3 / 16^{11}\right)$

\$ GLOVEBOX Stainless steel (3/32")

$\$$ MODEL TOP (GB roof $+100 \mathrm{~cm}$ )

$\$$ MODEL LEFT SIDE (GB side $+100 \mathrm{~cm}$ )

\$ GLOVEBOX left surface

\$ GLOVEBOX stainless steel (3/32")

GLOVEBOX Lead Shield (3/16")

GLOVEBOX Stainless steel (3/32")

\$ GLOVEBOX right inside surface

\$ GLOVEBOX stainless steel (3/32")

\$ GLOVEBOX Lead Shield (3/16")

\$ GLOVEBOX Stainless Steel (3/32")
\$ MODEL RIGHT SIDE (GB side +75")

$\$$ MODEL BACK SIDE (GB back $+100 \mathrm{~cm}$ )

$\$$ GLOVEBOX back surface

$\$$ GLOVEBoX Stainless steel (3/32")

$\$$ GLOVEBOX Lead Shield (3/16")

$\$$ GLOVEBOX stainless Steel (3/32")

$\$$ GLOVEBOX front inside surface

$\$$ GLOVEBOX stainless steel (3/32")

$\$$ GLOVEBOX Lead Shield (3/16")

py 82.7881 


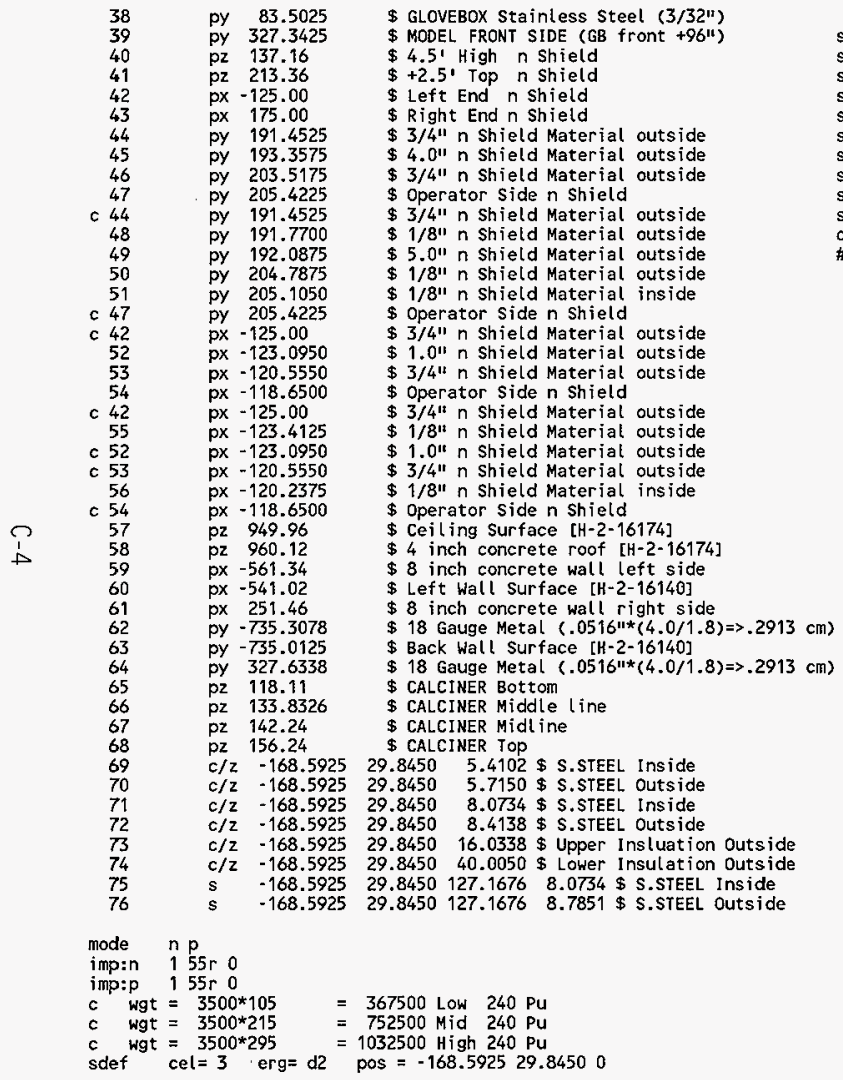

$\mathrm{rad}=\mathrm{d} 3$ ext $=\mathrm{d} 4 \quad$ axs $=0001$ wgt $=1032500$.

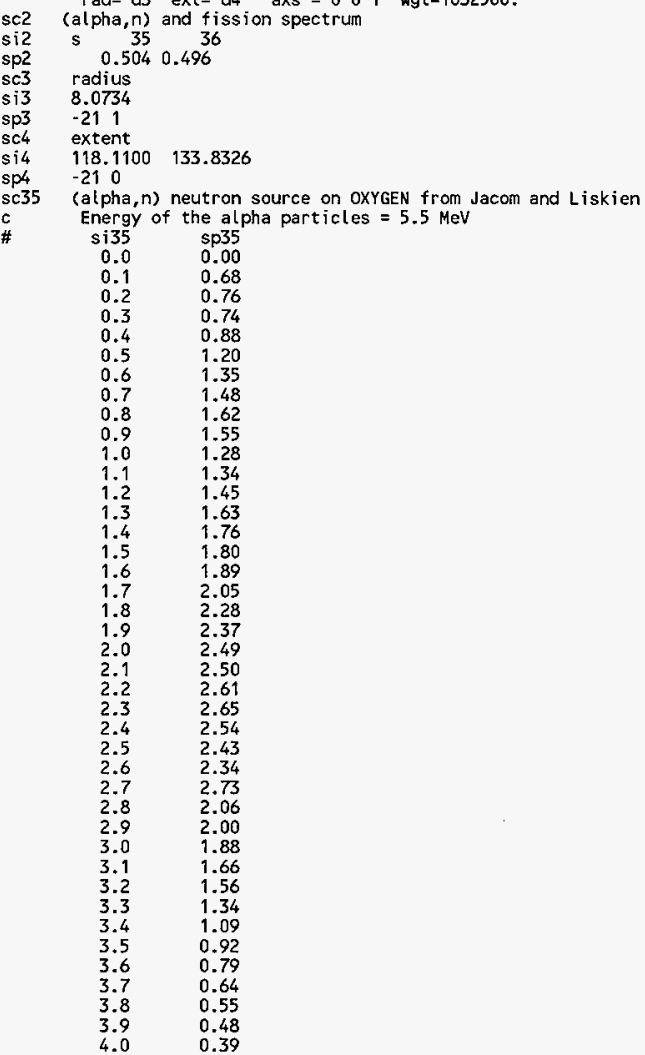


$4.1 \quad 0.35$

4.20 .23

$4.3 \quad 0.18$

$4.5 \quad 0.5$

sc36 spontaneous fission source spectrum (Pu240)

$\begin{array}{llllll}\text { sp3.6 } & -3 & 0.799 & 4.903 & \$ \text { Watt Fisson spectrum }\end{array}$

$94238.50 c-0.00027994239 .55 c-0.124257 \quad 94240.50 c-0.027689$

$94241.50 c-0.001894 \quad 94242.50 c-0.001133 \quad 95241.50 c-0.002778$

$7014.50 c-0.099011 \quad 8016.50 c-0.693830 \quad 1001.50 c-0.049129$

$\mathrm{mt1} \quad$ lwtr.01t

$94238.50 c-0.00008994239 .55 c-0.82309094240 .50 c-0.054516$

$94241.50 c-0.00231694242 .50 c-0.000356 \quad 95241.50 c-0.001603$

$8016.50 \mathrm{c}-0.118030 \$$ PuO2 for Low 240 Weight \% [CALCINE2.WQ1]

$\mathrm{m} 3$

$\begin{array}{lllll}94238.50 c & -0.001312 & 94239.55 c-0.748841 & 94240.50 c-0.108876\end{array}$

$8016.50 c-0.117971$ \$ PuO2 for Med 240 Height \% [CALCINE2.WQ1]

$\mathrm{m} 4$

$94238.50 c-0.00155904239 .55 c-0.693581 \quad 94240.50 c-0.154553$

$94241.50 c-0.010569 \quad 94242.50 c-0.006324 \quad 95241.50 c-0.015507$

$8016.50 \mathrm{c}-0.117907 \$$ PU02 for High 240 Weight \% [CALCINE2. WQ1]

$14000.50 c-0.376753 \quad 5010.50 c-0.007986 \quad 5011.55 c-0.032386$

$11023.50 c-0.029674 \quad 13027.50 c-0.012173 \quad 19000.50 c-0.000830$

$8016.50 \mathrm{c}-0.540198 \$ 7740$ PYREX TYPe I Glass [CALCINE1. WQ1]

mo $26000.55 \mathrm{c}-0.74 \quad 24000.50 \mathrm{c}-0.18 \quad 28000.50 \mathrm{c}-0.08 \$ \mathrm{SS} 304 \mathrm{~L}$. Flanges

$m 7 \quad 26000.55 c-0.98 \quad 28000.50 c-0.02 \quad \$$ Stainless Steel Glovebox

$m 8 \quad 82000.50 c-1.00$

$26000.55 c-1$

\$ Stainless Steel Glovelox
$\$$ Lead Glovebox Shield

$\$$ ss 310 S

$7014.50 c-.78084 \quad 8016.50 c-0.20946 \quad 18000.35 c-0.00934$ $1001.50 \mathrm{C}-.00036 \$ \mathrm{Air}$ \begin{tabular}{ccccc} 
mt10 & lwtr.01t & & \multicolumn{2}{c}{$\$$ hydrogen in liquid, gas } \\
m11 & $13027.50 \mathrm{c}-.034$ & $20000.50 \mathrm{c}-0.044$ & $26000.55 \mathrm{c}-0.014$ \\
& $1001.50 \mathrm{c}-.001$ & $8016.50 \mathrm{c}-0.532$ & $14000.50 \mathrm{c}-0.337$
\end{tabular}

$11023.50 \mathrm{c}-.029$ Concrete (common Portland) ARH-600 II.F.1-3

mt11 poly.01t

$\$$ hydrogen in sol id

m12 $6000.50 c-0.5925 \quad 5010.50 c-0.0590 \quad 5011.55 c-0.2410$ \$ Neutron $1001.50 c-0.0854 \quad 14000.50 c-0.0101 \quad 8016.50 c-0.0076 \$$ Shield $26000.55 c-0.0038 \quad 13027.50 c-0.0004 \quad 25055.50 c-0.0002 \$ 210$

mt12 poly.01t grph.01t \$ hydrogen, carbon in solid (1.19 g/cm3)

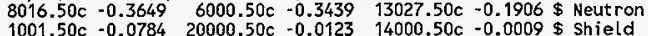
$\begin{array}{lllll}1001.50 c-0.0784 & 20000.50 c & -0.0123 & 14000.50 c-0.0009 & \text { Shiel }\end{array}$

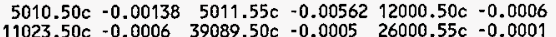

$19000.50 \mathrm{c}-0.000116032 .50 \mathrm{c}-0.0001 \$ Y 89$ subst $i$ tuted for $\mathrm{Sr}$.

$\mathrm{mt} 13$

$\mathrm{m} 14$

$\mathrm{mt} 14$

$\mathrm{m} 15$

$\mathrm{m} 16$

$\mathrm{mt} 16$

c de4 Photon dose conversion factors from ANS 6.1.1 199 poly.01t grph.01t \$ hydrogen, carbon in solid $\left(1.4 \mathrm{~g} / \mathrm{cm}^{3}\right)$ $6000.50 c-0.599848 \quad 1001.50 c-0.080538 \quad 8016.50 c-0.319614$ \& Lucite poly.01t grph.01t \$ hydrogen in solid $(1.185 \mathrm{~g} / \mathrm{cm} 3)$ Lucite $8016.50 c-0.364913027 .50 c-0.190614000 .50 c-0.0009 \$ .387 \mathrm{~g} / \mathrm{cc}$ $6000.50 c-0.7557511001 .50 c-0.0554918016 .50 c-0.188758$ \$ Lexan poly.01t grph.01t \$ hydrogen in solid $(1.2 \mathrm{~g} / \mathrm{cm} 3)$ Lexan

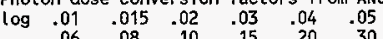

$\begin{array}{cccccc}.06 & .08 & .08 & .15 & .04 & .05 \\ .40 & .50 & .60 & .80 & 1.0 & 1.5 \\ 2.0 & 3.0 & 4.0 & 5.0 & 6.0 & 8.0\end{array}$

df4

$10.0 \quad 12.0$

og $2.232 e-5 \quad 5.652 e-5 \quad 8.568 e-5 \quad 1.184 e-4 \quad 1.314 e-4 \quad 1.382 e-4$

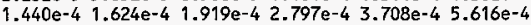

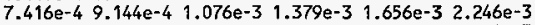
$2.758 \mathrm{e}-3 \quad 3.672 \mathrm{e}-3 \quad 4.500 \mathrm{e}-3 \quad 5.292 \mathrm{e}-3 \quad 6.012 \mathrm{e}-3 \quad 7.488 \mathrm{e}-3$ $8.892 \mathrm{e}-31.040 \mathrm{e}-2$

fc4 Photon Dose behind Neutron Shield, Right Side Leak Around

f $4: \mathrm{p} \quad 45 \quad$ \$ Operator behind Neutron Shield

cf $26 \quad \$$ Flagging Cells for leak-through cf 4 ing 26

Photon dose conversion factors from ANS 6.1.1 1991

de14 log $\begin{array}{llllll}.01 & .015 & .02 & .03 & .04 & .05\end{array}$

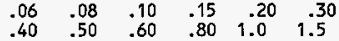

$\begin{array}{cccccc}.40 & .50 & .60 & .80 & 1.0 & 1.5 \\ 0.0 & 3.0 & 4.0 & 5.0 & 6.0 & 8.0\end{array}$

$10.0 \quad 12.0$

df $14 \quad \log 2.232 e-5 \quad 5.652 e-5 \quad 8.568 e-5 \quad 1.184 e-4 \quad 1.314 e-4 \quad 1.382 e-4$ $1.440 \mathrm{e}-4 \quad 1.624 \mathrm{e}-4 \quad 1.919 \mathrm{e}-4 \quad 2.797 \mathrm{e}-4 \quad 3.708 \mathrm{e}-4 \quad 5.616 \mathrm{e}-4$ $7.416 e-40.144 e-41.076 e-31.379 e-31.656 e-32.246 e-3$ $2.758 e-33.672 e-34.500 e-35.292 e-36.012 e-37.488 e-3$ $8.892 \mathrm{e}-3$ 1.040e-2

fc14 Photon Dose behind Neutron Shield, Front Lower $n$ Shield Leak Through f14:p 45 \$ Operator behind Neutron Shield

31 \$ Flagging Cells for leak-through

skyshine

$c$ Photon dose conversion factors from ANS 6.1.1 1991

de24 $\log \begin{array}{llllll}01 & .015 & .02 & .03 & .04 & .05\end{array}$

$\begin{array}{llllll}.06 & .08 & .10 & .15 & .20 & .050 \\ .40 & .50 & .60 & .80 & 1.0 & 1.5\end{array}$

$\begin{array}{llllll}2.0 & 3.0 & 4.0 & 5.0 & 6.0 & 8.0\end{array}$

$10.0 \quad 12.0$

df24 log $2.232 \mathrm{e}-5 \quad 5.652 \mathrm{e}-5 \quad 8.568 \mathrm{e}-5 \quad 1.184 \mathrm{e}-4 \quad 1.314 \mathrm{e}-4 \quad 1.382 \mathrm{e}-4$

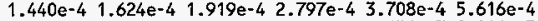

$7.440 e-41.624 e-41.919 e-42.797 e-43.708 e-45.616 e-4$

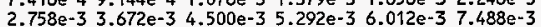

$8.892 \mathrm{e}-3$ 1.040e-2

fc24 Photon Dose behind Neutron Shield, Front Upper $n$ shield Leak Through f24:p 45 \$ Operator behind Neutron shield

cf24 34 \$ Flagging Cells for leak-through,

skyshine

de34 Photon dose conversion factors from ans 6.1.1 1991

$\begin{array}{llllll}.01 & .015 & .02 & .03 & .04 & .05 \\ .06 & .08 & .10 & .15 & .20 & .30\end{array}$

$\begin{array}{llllll}.06 & .08 & .10 & .15 & .20 & .30 \\ .40 & .50 & .60 & .80 & 1.0 & 1.5\end{array}$

$\begin{array}{llllll}2.0 & 3.0 & 4.0 & 5.0 & 6.0 & 8.0\end{array}$

$10.0 \quad 12.0$

$\log 2.232 e-5 \quad 5.652 e-5 \quad 8.568 e-5 \quad 1.184 e-4 \quad 1.314 e-4 \quad 1.382 e-4$

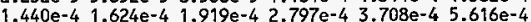

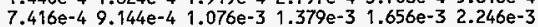

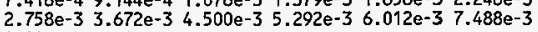
$8.892 \mathrm{e}-3 \quad 1.040 \mathrm{e}-2$

fc34 Photon Dose behind Neutron Shield, Left Lower $n$ shield Leak Through $f 34: \mathrm{p} \quad 45 \quad$ \$ Operator behind Neutron Shield

$\mathrm{cf3} 4^{\mathrm{p}}$

\$ Flagging Cells for leak-through, 
skyshine

c Photon dose conversion factors from ANS 6.1.1 1991

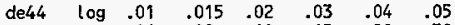

$\begin{array}{cccccc}.06 & .08 & .10 & .15 & .20 & .30 \\ .40 & .50 & .60 & .80 & 1.0 & 1.5 \\ 2.0 & 3.0 & 4.0 & 5.0 & 6.0 & 8.0\end{array}$

$2.0 \quad 3.0 \quad 4.0 \quad 5.0 \quad 6.0 \quad 8.0$

10.012 .0

df44 $\log 2.232 e-5 \quad 5.652 e-5 \quad 8.568 e-5 \quad 1.184 e-4 \quad 1.314 e-4 \quad 1.382 e-4$

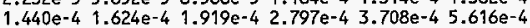

$\begin{array}{llllll}1.440 e-4 & 1.624 \mathrm{e}-4 & 1.919 \mathrm{e}-4 & 2.797 \mathrm{e}-4 & 3.708 \mathrm{e}-4 & 5.616 \mathrm{e}-4 \\ 7.416 \mathrm{e}-4 & 9.144 \mathrm{e}-4 & 1.076 \mathrm{e}-3 & 1.379 \mathrm{e}-3 & 1.656 \mathrm{e}-3 & 2.246 \mathrm{e}-3\end{array}$

$2.758 \mathrm{e}-3 \quad 3.672 \mathrm{e}-3 \quad 4.500 \mathrm{e}-3 \quad 5.292 \mathrm{e}-3 \quad 6.012 \mathrm{e}-3 \quad 7.488 \mathrm{e}-3$ $8.892 \mathrm{e}-3$ 1.040e-2

fc44 Photon Dose behind Neutron Shield, Left Upper n Shield leak Through df84

f44:p 45 \$ Operator behind Neutron Shield

cf44 43 \$ Flagging Cells for leak-through,

skyshine

c Photon dose conversion factors from ANS 6.1 .11991

de54 $\log \begin{array}{llllll}.01 & .015 & .02 & .03 & .04 & .05\end{array}$

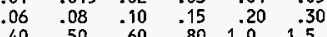

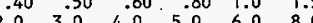

$2.0 \quad 3.0$

df54 $\log 2.232 e-5 \quad 5.652 e-5 \quad 8.568 e-5 \quad 1.184 e-4 \quad 1.314 e-4 \quad 1.382 e-4$

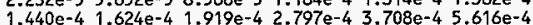

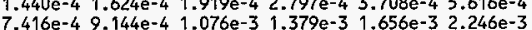

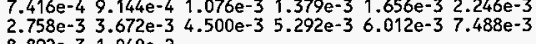
$8.892 \mathrm{e}-31.040 \mathrm{e}-2$

fc54 Photon Dose behind Neutron Shield, Roof Reflection

f54:P 45 \$ Operator behind Neutron Shield

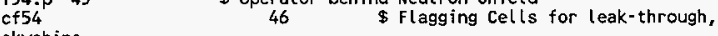

skyshine

Photon dose conversion factors from ANS 6.1 .11991

de64 log $\begin{array}{llllll}.01 & .015 & .02 & .03 & .04 & .05\end{array}$

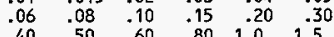

$2.40 \quad .50 \quad .60 \quad 5.00 \quad 1.00 \quad 1.5$

$10.0 \quad 12.0$

df64 $\log 2.232 \mathrm{e}-5 \quad 5.652 \mathrm{e}-5 \quad 8.568 \mathrm{e}-5 \quad 1.184 \mathrm{e}-4 \quad 1.314 \mathrm{e}-4 \quad 1.382 \mathrm{e}-4$

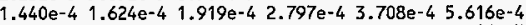
$7.416 e-4 \quad 9.144 e-4 \quad 1.076 e-3 \quad 1.379 e-3 \quad 1.656 e-3 \quad 2.246 e-3$ $2.758 \mathrm{e}-3 \quad 3.672 \mathrm{e}-3 \quad 4.500 \mathrm{e}-3 \quad 5.292 \mathrm{e}-3 \quad 6.012 \mathrm{e}-3 \quad 7.488 \mathrm{e}-3$ $8.892 \mathrm{e}-3 \quad 1.040 \mathrm{e}-2$

fc64 Photon Dose behind Neutron Shield, Steel Wall Back Reflection f64:p 45 \$ Operator behind Neutron Shield \$ Operator behind Neutron Shield
cf64

Photon dose conversion factors from ANS 6.1.1 1991

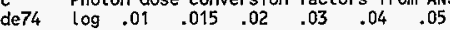

$\begin{array}{llllll}.06 & .08 & .10 & .15 & .04 & .05 \\ .40 & .50 & .60 & .80 & 1.0 & 1.5\end{array}$

$\begin{array}{cccccc}.40 & .50 & .60 & .80 & 1.0 & 1.5 \\ 2.0 & 3.0 & 4.0 & 5.0 & 6.0 & 8.0\end{array}$

10.012 .0

$\begin{array}{lllllll}\text { df7 } \quad \log \quad 2.232 e-5 & 5.652 e-5 & 8.568 e-5 & 1.184 e-4 & 1.314 e-4 & 1.382 e-4\end{array}$

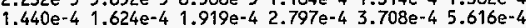

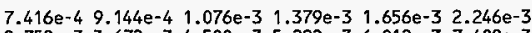
$2.758 \mathrm{e}-3 \quad 3.672 \mathrm{e}-3 \quad 4.500 \mathrm{e}-3 \quad 5.292 \mathrm{e}-3 \quad 6.012 \mathrm{e}-3 \quad 7.488 \mathrm{e}-3$ $8.892 \mathrm{e}-3 \quad 1.040 \mathrm{e}-2$

fc74 Photon Dose behind Neutron Shield, Concrete Floor Effect

f74:P $45 \quad \$$ Operator behind Neutron Shield

Photon dose conversion factors from ANS 6.1 .11991

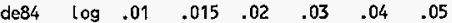

$\begin{array}{llllll}.06 & .08 & .10 & .15 & .20 & .050\end{array}$

$\begin{array}{llllll}.00 & 3.0 & 4.0 & 5.0 & 6.0 & 8.0\end{array}$

$10.0 \quad 12.0$

$\log 2.232 \mathrm{e}-5 \quad 5.652 \mathrm{e}-5 \quad 8.568 \mathrm{e}-5 \quad 1.184 \mathrm{e}-4 \quad 1.314 \mathrm{e}-4 \quad 1.382 \mathrm{e}-4$ $1.440 \mathrm{e}-4 \quad 1.624 \mathrm{e}-4 \quad 1.919 \mathrm{e}-4 \quad 2.797 \mathrm{e}-4 \quad 3.708 \mathrm{e}-4 \quad 5.616 \mathrm{e}-4$

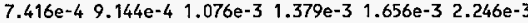
$2.758 \mathrm{e}-3 \quad 3.672 \mathrm{e}-3 \quad 4.500 \mathrm{e}-3 \quad 5.292 \mathrm{e}-3 \quad 6.012 \mathrm{e}-3 \quad 7.488 \mathrm{e}-3$ $8.892 \mathrm{e}-31.040 \mathrm{e}-2$

fc84 Photon Dose behind Neutron Shield, Sum of All Cells FLAGGING!!

f84:P $45 \quad$ \$ Operator behind Neutron Shield

cf84 $26 \quad 31 \quad 34 \quad 40 \quad 43 \quad 46 \quad 51 \quad 56$ \$ Flagging Cells

Neutron dose conversion factors from ANS 6.1.1 1991

de $0 \quad 2.50 \mathrm{e}-08 \quad 1.00 \mathrm{e}-07 \quad 1.00 \mathrm{e}-06 \quad 1.00 \mathrm{e}-05 \quad 1.00 \mathrm{e}-04 \quad 1.00 \mathrm{e}-03$

$1.00 \mathrm{e}-02 \quad 2.00 \mathrm{e}-02 \quad 5.00 \mathrm{e}-02 \quad 1.00 \mathrm{e}-01 \quad 2.00 \mathrm{e}-01 \quad 5.00 \mathrm{e}-01$

$\begin{array}{llllll}1.00 \mathrm{e}-02 & 2.00 \mathrm{e}-02 & 5.00 \mathrm{e}-02 & 1.00 \mathrm{e}-01 & 2.00 \mathrm{e}-01 & 5.00 \mathrm{e}-01 \\ 1.00 \mathrm{e}+00 & 1.50 \mathrm{e}+00 & 2.00 \mathrm{e}+00 & 3.00 \mathrm{e}+00 & 4.00 \mathrm{e}+00 & 5.00 \mathrm{e}+00\end{array}$

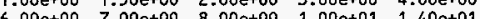

df0 $\quad 1.440 \mathrm{e}-03 \quad 1.584 \mathrm{e}-03 \quad 1.735 \mathrm{e}-03 \quad 1.606 \mathrm{e}-03 \quad 1.490 \mathrm{e}-03 \quad 1.379 \mathrm{e}-03$

$1.631 \mathrm{e}-03 \quad 2.113 \mathrm{e}-03 \quad 3.924 \mathrm{e}-03 \quad 7.128 \mathrm{e}-03 \quad 1.390 \mathrm{e}-02 \quad 3.132 \mathrm{e}-02$

$5.148 \mathrm{e}-02 \quad 6.588 \mathrm{e}-02 \quad 7.704 \mathrm{e}-02 \quad 9.504 \mathrm{e}-02 \quad 1.080 \mathrm{e}-011.177 \mathrm{e}-01$

$1.249 \mathrm{e}-01 \quad 1.314 \mathrm{e}-01 \quad 1.368 \mathrm{e}-01 \quad 1.476 \mathrm{e}-011.728 \mathrm{e}-01$

fc204 Neutron Dose behind Neutron Shield, Right Side Leak Around

f204:n 45 \$ Operator behind Neutron Shield

fm204 2.0 \$ Factor of 2 times the ANS 6.1.1 1991 standard.

cf204 26 \$ Flagging Cells for leak-through,

fc214 Neutron Dose behind Neutron Shield, Front Lower $n$ Shield Leak

Through

214:n 45 \$ Operator behind Neutron Shield

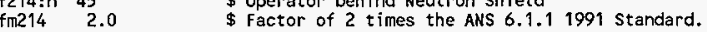

cf214 31 \$ Flagging Cells for leak-through,

skyshine

fc224 Neutron Dose behind Neutron Shield, front Upper $n$ Shield Leak

Through

f224: $45 \quad$ \$ Operator behind Neutron Shield

fm224 2.0 \$ Factor of 2 times the ANS 6.1.1 1991 standard.

cf224 $34 \quad \$$ flagging Cells for leak-through,

skyshine

fc234

Through

$\begin{array}{ll}f 234: n & 45 \\ f m 234 & 2.0\end{array}$

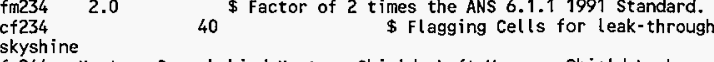

fc244 Neutron Dose behind Neutron Shield, Left Upper n Shield Leak

Through 
$\begin{array}{lr}f 244: n & 45 \\ f m 244 & 2.0\end{array}$

cf 244

skyshine

fc254 Neutron Dose behind Neutron Shield, Roof Reflection

fc254 Neutron Dose behind Neutron Shield, Roof Reflectic

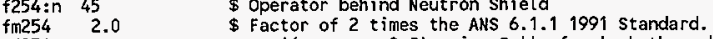

cf254 46 \$ Flagging Celis for leak-through

fc264 Neutron Dose behind Neutron Shield, Steel Wall Reflection

f264: $\mathrm{n} \quad 45$ \$ Operator behind Neutron Shield

fm264 $2.0 \quad$ \$ Factor of 2 times the ANS 6.1 .11991 standard.

cf 264

skyshine

fc274 Neutron Dose behind Neutron Shield, Sum of All Cells FLAGGING!!

$\$$ Operator behind Neutron Shield

fm274 2.0 Factor of 2 times the ANS 6.1 .11991 standard.

2 flagging cells for leak-through.

cf 274 .

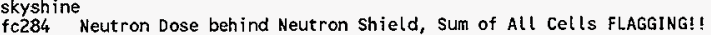

fc284 Neutron Dose behind Neutron Shield, Sum of All
f284:n 45

$\begin{array}{lll}\text { f284:n } & 45 & \text { \$ Operator behind Neutron Shield } \\ \text { fm284 } & 2.0 & \text { \$ Factor of } 2 \text { times the ANS } 6.1 .11991 \text { standard. }\end{array}$

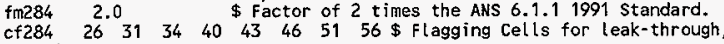

skyshine

fc5 Neutron dose at $30 \mathrm{~cm}$ from outside surface of Calciner

c Total Dist from outside $=30.0+29.8450+40.0050=69.85 \mathrm{~cm}$ $f 5: n \quad-168.5925 \quad 69.85 \quad 125.9713 \quad 0$

fm5 2.0 \$ Factor of 2 times the ANS 6.1.1 1991 Standard.

fc15 Neution dose at $30 \mathrm{~cm}$ from outside surface of Glovebox

fc15 Neutron ios from outside $=30.0+83.5025=113.5025 \mathrm{~cm}$

f15: $\quad-168.5925113 .5025125 .9713$

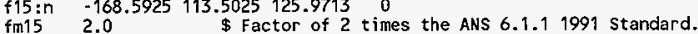

$\begin{array}{lll}\mathrm{fm} 15 & 2.0 \quad \text { Neutron dose at } 5 \text { feet from outside surface of Glovebox } \\ \mathrm{fc} 25 & \end{array}$

fc25 Neutron dose at 5 feet from outside surface of Glovebox
Total Dist from outside $=152.8+83.5025=235.9025 \mathrm{~cm}$

$\begin{array}{lll}c & \text { Total Dist from outside }=152.8+ \\ \mathrm{f} 25: \mathrm{n} & -168.5925 & 235.9025 \\ \text { 125.9713 } 0\end{array}$

fm25 2.0 \$ Factor of 2 times the ANS 6.1 .11991 standard.

fc35 Neutron dose at 6 in. in front of lower $n$ Shield

c Total Dist from outside $=191.4525-6 \star 2.54=176.2125 \mathrm{~cm}$

$\mathrm{f} 35 \mathrm{n} \quad \mathrm{n} \quad \mathrm{O} 176.2125 \quad 91.44 \quad 0$

$\mathrm{fm} 352.0 \quad$ \$ Factor of 2 times the ANS 6.1.1 1991 standard.

fc45 Neutron dose in middle of outer 1/8" SST $n$ shield

$c$ Total Dist from outside $=(191.7700+192.0875) / 2=191.9288 \mathrm{~cm}$

f45: $\quad 0$ r $191.9288 \quad 91.44 \quad 0$

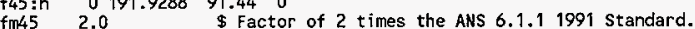

fm45 2.0 Neutron dose in middle of 5.0 "1 lower $n$ Shield

$\mathrm{fc} 55$ Neutron dose in middle of 5.011
Total Dist from outside $=(192.0875+204.7875) / 2=198.4375 \mathrm{~cm}$

$\begin{array}{cc}c & \text { Total Dist from outs ide } \\ \text { f55:n } \quad 0198.4375 \quad 91.44 \quad 0\end{array}$

fm55 2.0 \$ Factor of 2 times the ANS 6.1 .11991 standard. fc65 Neutron dose in middle of inner $1 / 8$ " Stainless steel $n$ Shield

c Total Dist from outside $=(204.7875+205.1050) / 2=204.9462 \mathrm{~cm}$

f65:n $\quad 0204.9462 \quad 91.44 \quad 0$

fm65 2.0 \$ Factor of 2 times the ANS 6.1.1 1991 standard.

fc75 Neutron dose at $6 \mathrm{in}$. behind inside lower $\mathrm{n}$ Shield

c f75:n

f $\mathrm{f} 75$

$8176.2125182 .88 \quad 0$

2.0 Factor of 2 times the ANS 6.1.1 1991 Standard.

f95 Neutron dose in middle of outer 3/4" glass n Shield

c Total Dist from outside $=(191.4525+193.3575) / 2=192.4050 \mathrm{~cm}$

f95:n $\quad 0 \quad 192.4050 \quad 182.88 \quad 0$

2.0 \$ Factor of 2 times the ANS 6.1.1 1991 Standard.

fc105 Neutron dose in middle of 4.0" upper $n$ Shield

c Total Dist from outside $=(193.3575+203.5175) / 2=198.4375 \mathrm{~cm}$

f105:n 0 198.4375 182.88 0

fm105 2.0 \$ Factor of 2 times the ANs 6.1 .11991 standard. fc115 Neutron dose in middle of inner $3 / 4^{\prime \prime}$ glass $n$ shield

$c$ Total Dist from outside $=(203.5175+205.4225) / 2=204.4700 \mathrm{~cm}$ f115:n $0204.4700182 .88 \quad 0$

fm115 2.0 \$ Factor of 2 times the ANS 6.1.1 1991 standard. fc125 Neutron dose at 6 in. behind inside upper $n$ shield

Total Dist from outside $=205.4225+6 * 2.54=220.6225 \mathrm{~cm}$

f125:n 0220.6625182 .88 o

fmi25 2.0 \$ Factor of 2 times the ANS 6.1 .11991 standard. print $10 \quad 110 \quad 120$

ctme 180.0

nps $\quad 1.0 \mathrm{e} 5$

CCA08AP.INP Vertical Calciner, $3.5 \mathrm{~kg} \mathrm{Pu}$, gamma ray calculation

PHOTON DOSe Rate Calcs for calciner CCA08aP

c CALCINER PU02 Gama Source is in Cell 3

c N Shield Lucite $-1.185 \mathrm{~g} / \mathrm{cm}^{3}-4$ inches top, 5 inches bottom

Lower Shields are $1 / 8$ inch of Stainless Steel (both sides)

Lower Shields are $1 / 8$ inch of Stainless Steel (both sides)
Upper Shields are $3 / 4$ inch of Glass (no Boron)(both sides)

Full Room Model with concrete Walls, Roof and Metal Partitions

c $x x-x x$ yy -yy $22-z z \quad r r-r r \$$

$110-1.29 \mathrm{e}-3 \quad 65-66 \quad-69 \$$ Air Gap

$\begin{array}{llll}2 & 7 & -7.84\end{array}$

$\begin{array}{lll}3 & 4 & -5.00\end{array}$

$410-1.29 \mathrm{e}-3$

5 7 - 7.84

$610-1.29 \mathrm{e}-3$

$7 \quad 7 \quad-7.84$

$815-0.387$

$910-1.29 \mathrm{e}-3$

$107 \begin{array}{lll}10 & 7 & -7.84 \\ 11 & 6 & -0.793\end{array}$

$\begin{array}{rrr}11 & 6 & -0.793 \\ 12 & 10 & -1.29 e-3\end{array}$

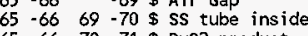

$\begin{array}{lllll}65 & -66 & 70 & -71 & \$ \\ 66 & -67 & -75 & \$ \text { PuO2 product }\end{array}$

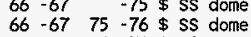

$66-67 \quad 76-71$ \$ Air Annulus

$\begin{array}{lllll}65 & -67 & 71 & -72 & \$\end{array}$ SS tube outs ide

$\begin{array}{lllll}65 & -67 & 72 & -74 & \text { \$ Lower Insulation }\end{array}$

$67-68 \quad-71$ \$ Air Gap

$\begin{array}{lllll}67 & -68 & 71 & -72 & \$\end{array}$ SS tube outside

$\begin{array}{lllll}67 & -68 & 72 & -73 & \$\end{array}$ Upper Insulation

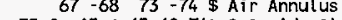

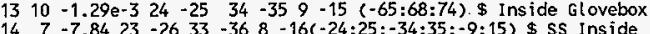
$14 \quad 7-7.8423-26 \quad 33-36 \quad 8-16(-24: 25:-34: 35:-9: 15)$ \$ SS Inside

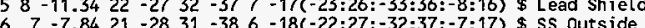




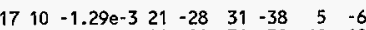

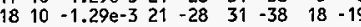

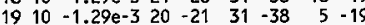

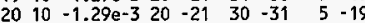

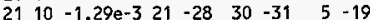

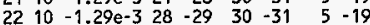

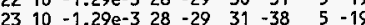

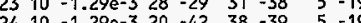

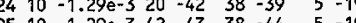

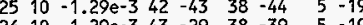

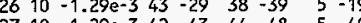

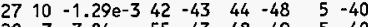
$\begin{array}{lllllllll}28 & 7 & -7.84 & 55 & -43 & 48 & -49 & 5 & -40\end{array}$ $\begin{array}{lllllllll}29 & 14 & -1.185 & 52 & -43 & 49 & -50 & 5 & -40\end{array}$ $\begin{array}{lllllllll}30 & 7 & -7.84 & 53 & -43 & 50 & -51 & 5 & -40\end{array}$

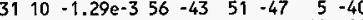
$\begin{array}{lllllllll}32 & 15 & -2.14 & 42 & -43 & 44 & -45 & 40 & -41\end{array}$ $\begin{array}{lllllllll}33 & 14 & -1.185 & 52 & -43 & 45 & -46 & 40 & -41\end{array}$ $\begin{array}{lllllllll}34 & 15 & -2.14 & 53 & -43 & 46 & -47 & 40 & -41\end{array}$

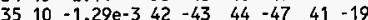

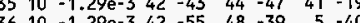
$\begin{array}{llll}48 & -39 & 5 & -40\end{array}$

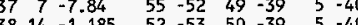

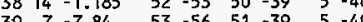
$\begin{array}{lllllllll}39 & 7 & -7.84 & 53 & -56 & 51 & -39 & 5 & -40\end{array}$

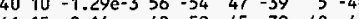
$\begin{array}{lllllllll}41 & 15 & -2.14 & 42 & -52 & 45 & -39 & 40 & -41\end{array}$

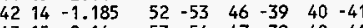
$\begin{array}{llllllllll}43 & 15 & -2.14 & 53 & -54 & 47 & -39 & 40 & -41 \\ 1 & 44 & 10 & -1.29 e-3 & 42 & -54 & 47 & -39 & 41 & -19\end{array}$

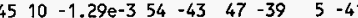

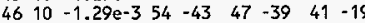

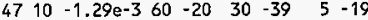

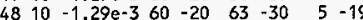

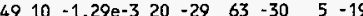

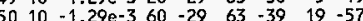

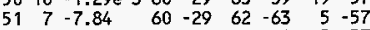

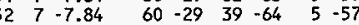
$\begin{array}{lllllllll}53 & 11 & -2.30 & 59 & -60 & 62 & -64 & 5 & -57\end{array}$

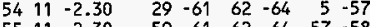
$\begin{array}{rrrrrrrrr}55 & 11 & -2.30 & 59 & -61 & 62 & -64 & 57 & -58 \\ 56 & 11 & -2.30 & 59 & -61 & 62 & -64 & 4 & -5\end{array}$ 570 61:-59: $64:-62:-4: 58$
Inder Glovebox

Glovebox

- Over Glovebox

Left Glovebox

Behind Glovebox

R-B Glovebox

$\$$ Right Glovebox

$\$$ Front Glovebox

$\$$ Front Glovebox

$\$$ Front Glovebox

$\$$ Bot Fr Outside air

$\$$ Bot Fr Outer 1/8

$\$$ Bot Fr Middle $5.0^{\text {r }}$

$\$$ Bot Fr Inside $1 / 8^{\prime \prime}$

$\$$ Bot Fr Inside air

\$ Top Fr Outer 3/4"

\$ Top Fr Middle $4.0^{\prime \prime}$

$\$$ Top Fr Inside $3 / 4^{\prime \prime}$

$\$$ Above Shield

$\$$ Bot L Outside air

$\$$ Bot L Middle 1.0"

Bot L Inside 1/8"

Bot L Inside air

Top L Outer 3/4"

Top L Middle $1.0^{\prime \prime}$

$\$$ Top L Inside 3/4"

$\$$ above shield

$\$$ Behind $n$ shield

$\$$ Behind $n$ shield

$\$$ Room, Left Side

$\$$ Room, Left Back

Room, Back Part

Room to Ceiling

ss Wall Back

SS Wall Front

s.

$\$$ Concrete Wall Left

$\$$ Concrete Wall

Concrete floor

$\$$ OUTSIDE WORLD

$\begin{array}{rrr}1 & \mathrm{cz} & 7.7610 \\ 2 & \mathrm{cz} & 8.4534 \\ 3 & \mathrm{cz} & 14.2875 \\ 4 & \mathrm{pz} & -60.9600 \\ 5 & \mathrm{pz} & 0.0000 \\ 6 & \mathrm{pz} & 86.3600 \\ 7 & \mathrm{pz} & 86.5981 \\ 8 & \mathrm{pz} & 87.0744 \\ 9 & \mathrm{pz} & 87.3125 \\ 10 & \mathrm{pz} & 171.4500 \\ 11 & \mathrm{pz} & 171.7200 \\ 12 & \mathrm{pz} & 224.5663\end{array}$

$\$$ FEED TANK O.R. - (5/16"1-0.040"1)

FEED TANK O.R.

\$ FeEd tank flange o.R.

$\$$ CONCRETE FLOOR bottom (24" thick)

$\$$ CONCRETE FLOOR surface

$\$$ GLOVEBOX bottom surface

\$ GLOVEBOX Stainless Steel (3/32")

GLOVEBOX Lead Shield (3/16")

$\$$ GLOVEBOX Stainless Steel (3/32")

$\$$ FEED TANK Bottom Flange bottom

$\$$ FEED TANK Bottom Flange to

$\$$ FEED TANK Solution level (10 L)

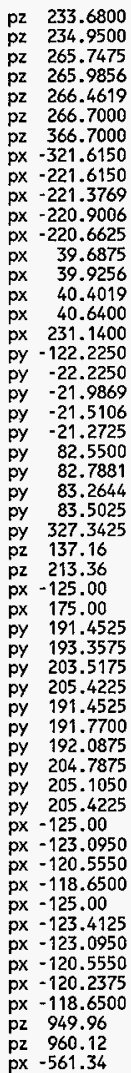

$\$$ FEED TANK TOP Flange bottom

$\$$ FEED TANK TOP Flange top

$\$$ GLOVEBOX top inside surface

$\$$ GLOVEBOX Stainless Steel $\left(3 / 32^{12}\right)$

$\$$ GLOVEBOX Lead Shield (3/16")

$\$$ GLOVEBOX Stainless Steel (3/32'x)

$\$$ MODEL TOP (GB roof $+100 \mathrm{~cm}$ )

$\$$ MODEL LEFT SIDE (GB side $+100 \mathrm{~cm}$ )

\$ GLOVEBOX left surface

\$ GLOVEBOX Stainless Steel (3/32"x)

\$ GLOVEBOX Lead Shield (3/16")

$\$$ GLovEBOX Stainless Steel (3/32"1)

$\$$ GLOVEBOX right inside surface

$\$$ GLOVEBOX Stainless steel $\left(3 / 32^{12}\right)$

$\$$ GLOVEBOX Lead Shield (3/16")

$\$$ GLOVEBOX Stainless Steel $\left(3 / 32^{12}\right)$

\$ MODEL RIGHT SIDE (GB side +75")

$\$$ MODEL BACK SIDE (GB back $+100 \mathrm{~cm}$ )

\$ GLOVEBOX back surface

\$ GLOVEBOX Stainless Steel (3/32")

$\$$ GLOVEBOX Lead Shield (3/16")

$\$$ GLOVEBOX Stainless Steel (3/32"1)

$\$$ GLOVEBoX front inside surface

$\$$ GLOVEBOX Stainless steel $\left(3 / 32^{12}\right)$

\$ GLOVEBOX Lead Shield (3/16")

$\$$ GLOVEBOX Stainless Steel (3/321i)

$\$$ MODEL FRONT SIDE (GB front +96")

$\$ 4.51$ High $n$ Shield

$\$+2.5$ ' Top $n$ shield

$\$$ Left End $n$ Shield

$\$$ Right End $n$ Shield

$\$ 3 / 4 "$ n Shield Material outside

$\$ 4$.0" n Shield Material outside

$\$ 3 / 4 "$ n Shield Material outside

$\$$ operator side $n$ shield

$\$ 3 / 4 "$ n Shield Material outside

$\$ 1 / 8$ n $n$ Shield Material outside

$\$ 1 / 8^{\prime \prime}$ n Shield Material outside

$\$ 5.0^{\prime \prime}$ n Shield Material outside

$\$ 1 / 8 "$ n Shield Material outside

$\$ 1 / 8 "$ n Shield Material

$\$ 3 / 4^{\prime \prime} n$ Shield Material outside

$\$ 1.0 "$ n Shield Material outside

$\$ 3 / 4 "$ n Shield Material outside

$\$$ Operator Side $n$ Shield

$\$ 3 / 4 "$ n Shield Material outside

$\$ 1 / 8$ " n shield Material outside

$\$ 1$ " n shield Material outside

$\$ 1.0$ " n shield Material outside

$\$ 3 / 4 "$ n Shield Material outside

$\$ 1 / 8$ n shield Material in

$\$$ Operator side $n$ Shield

$\$$ Ceiling surface [H-2-16174]

$\$ 4$ inch concrete roof [H-2-16174]

$\$ 8$ inch concrete wall left side 


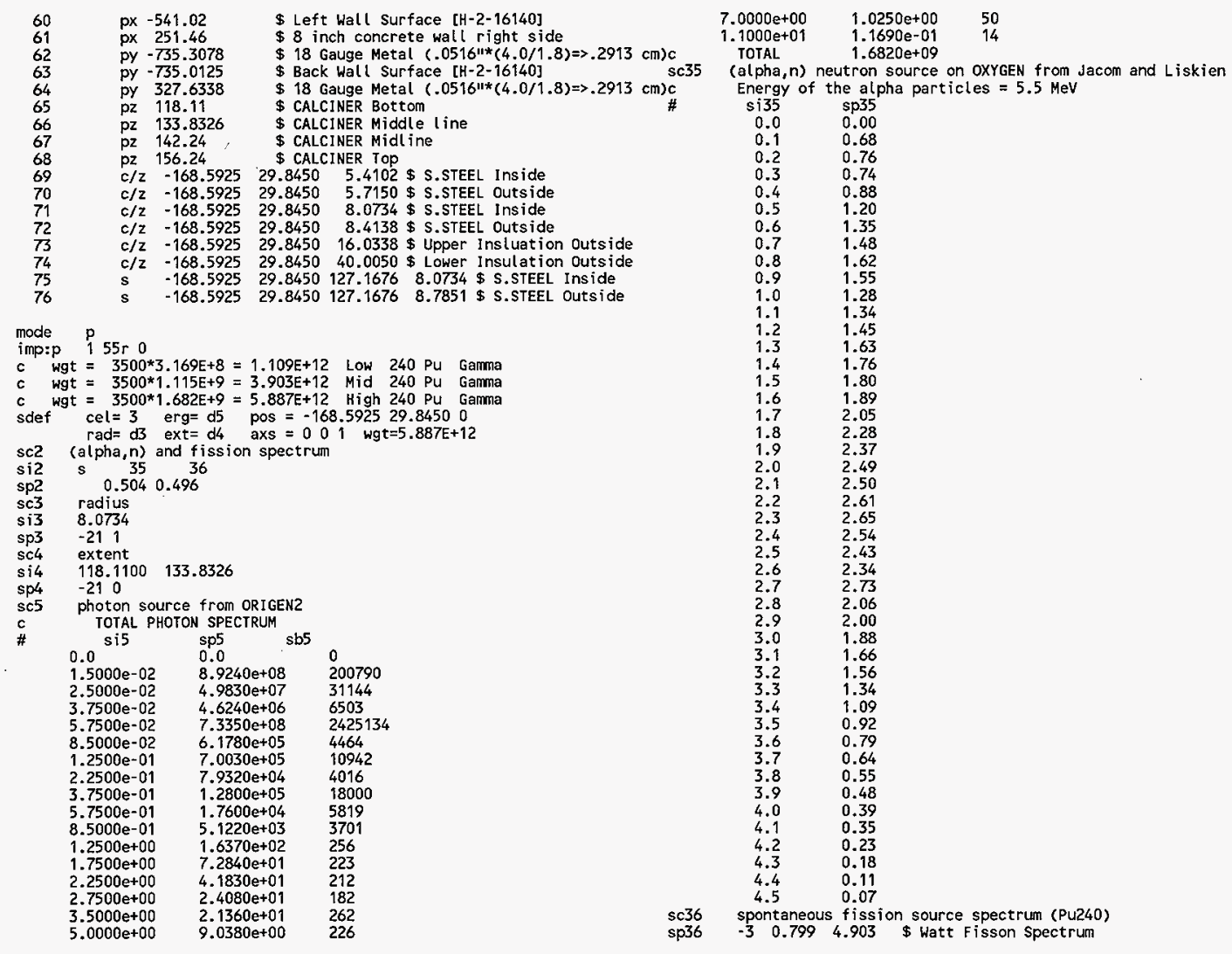


94238. -0.000279 94239. $-0.12425794240,-0.027689$ 94241. -0.001894 94242. -0.001133 95241. -0.002778 7014. $-0.0990118016 .-0.693830$ 1001. -0.049129 c mt lwtr.01t \$ hydrogen in liquid [CALCINE4. WQ1]

94238, $-0.00008994239,-0.823090$ 94240. -0.054516

94241. -0.00231694242 . $-0.00035695241 .-0.001603$

$\mathrm{m}^{3}$

94238. $-0.00131294239,-0.74884194240,-0.108876$

$94241 .-0.01049494242 .-0.00323695241 .-0.009270$

8016. -0.117971 \$ PuO2 for Med 240 Weight \% [CALCINE2. WQ1]

94238. -0.001559 94239. $-0.69358194240 .-0.154553$

94241 . $-0.01056994242,-0.00632495241 .-0.015507$
$8016 .-0.117907$ \$ PuO2 for High 240 Weight \% [CALCINE2. WQ1]

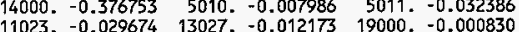

8016. $-0.540198 \$ 7740$ PYREX Type I Glass [CALCINE1.WQ1]

$26000,-0.7424000$. $-0.18 \quad 28000$. -0.08 \$ SS $304 \mathrm{~L}$ Flanges

26000 . $-0.9828000 .-0.02$ \$ Stainless steel Glovebox

$82000-1.00$

26000 . -1

26000. 1

$\$$ lead Glovebox Shield

$\$$ SS 310 S

meto. .00036 \$ Air

c mt10 litr.01t $\$$ hydrogen in liquid, gas

13027. -.034 20000. $-0.044 \quad 26000$. -0.014

$1001 .-001 \quad 8016 .-0.532$

11023. - .029 \$ Concrete (common Portland) ARH-600 I1.F.1-3

c mt11 poly.01t

$\$$ hydrogen in solid

5010. -0.0590 5011. -0.2410 \$ Neutron

1001. -0.0854 14000. -0.0101 8016. $-0.0076 \$$ Shield $\mathrm{g} / \mathrm{cm} 3)$ met12 poly 010

$\$$ hydrogen, carbon in solid $(1.19$

$\begin{array}{lllllll}\mathrm{m} 13 & 8016 & -0.3649 & 6000 & -0.3439 & 13027 & -0.1906 \text { \$ Neutron }\end{array}$ 1001. -0.0784 20000- 0.0123 14000 $-0.0009 \$$ Shield

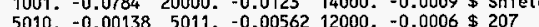

5010. -0.00138 5011. -0.00562 12000. -0.0006

$11023 .-0.0006$ 39089. -0.0005 26000. -0.0001 y 160 substituted for $\mathrm{sr}$ c mt13 poly.01t grph.01t \$ hydrogen, carbon in sol id $(1.4$ $\mathrm{g} / \mathrm{cm} 3)$

m14 6000, -0.599848 1001. -0.080538 8016. -0.319614 \$ Lucite c mt14 poly.01t grph.01t \$ hydrogen in solid $(1.185 \mathrm{~g} / \mathrm{cm} 3)$

$\begin{array}{llllll}\text { Lucite } & \text { 8016. }-0.3649 & 13027 & -0.1906 \quad 14000 & -0.0009 \$ .387 \mathrm{~g} / \mathrm{cc}\end{array}$

m15 $6000-0.755751,1001-0.0554918016,-0.188758$ Lexan

c mt16 poly.01t grph.01t \$ hydrogen in solid $(1.2 \mathrm{~g} / \mathrm{cm} 3)$

Lexan

Photon dose conversion factors from ANS 6.1 .11991

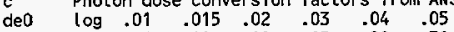

$\begin{array}{llllll}.06 & .08 & .10 & .15 & .02 & .30 \\ .40 & .50 & .60 & .80 & 1.0 & 1.5\end{array}$

$\begin{array}{llllll}2.0 & 3.0 & 4.0 & 5.0 & 6.0 & 8.0\end{array}$

$10.0 \quad 12.0$

dfo $\quad \log 2.232 e-5 \quad 5.652 e-5 \quad 8.568 e-5 \quad 1.184 e-4 \quad 1.314 e-4 \quad 1.382 e-4$

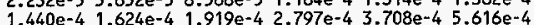

$\begin{array}{llllll}7.416 e-4 & 9.144 \mathrm{e}-4 & 1.076 \mathrm{e}-3 & 1.379 \mathrm{e}-3 & 1.656 \mathrm{e}-3 & 2.246 \mathrm{e}-3\end{array}$ $2.758 \mathrm{e}-3 \quad 3.672 \mathrm{e}-3 \quad 4.500 \mathrm{e}-3 \quad 5.292 \mathrm{e}-3 \quad 6.012 \mathrm{e}-3 \quad 3.488 \mathrm{e}-3$ $8.892 \mathrm{e}-3$ 1.040e-2

fc4 Photon Dose behind Neutron Shield, Right Side Leak Around

$f 4: p \quad 45 \quad \$$ Operator behind Neutron Shield

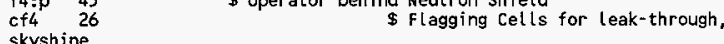

fc14 Photon Dose behind Neutron Shield, Front Lower $n$ Shield Leak Through

fc14 Photon Dose behind Neutron Shield, Front Lower $n$ Shield Leak Through
f14:p $45 \quad$ \$ Operator behind Neutron Shield

cf14 31

skyshine

fe24 Photon Dose behind Neutron Shield, Front Upper n Shield Leak Through

f24:P $45 \quad$ \$Operator behind Neutron Shield

cf24 $34 \quad \$$ Flagging Cells for leak-through,

skyshine

fc34 Photon Dose behind Neutron Shield, Left Lower $n$ shield Leak Through

$\begin{array}{lll}\text { f34:P } 45 & 40 & \$ \text { Operator behind Neutron Shield } \\ \text { cf34 } & \$ \text { Flagging Cells for leak-through, }\end{array}$

skyshine

fc44 Photon Dose behind Neutron Shield, Left Upper $\mathrm{n}$ Shield Leak Through

fc44 Photon Dose behind Neutron Shield, Left Upper $n$
f44:p 45
\$ Operator behind Neutron Shield

$\begin{array}{lll}\text { f44:P } 45 & \$ \text { Operator behind Neutron Shield } \\ \text { cf } 44 & 43 & \text { Flagging Cells for leak-through, }\end{array}$

skyshine

fC54 Photon Dose behind Neutron Shield, Roof Reflection

f54:p $45 \quad$ \$Operator behind Neutron Shield

cf54 $46 \quad \$$ Flagging cells for leak-through,

skyshine

fc64 Photon Dose behind Neutron Shield, steel Wall Back Reflection

f64:P 45 \$ Operator behind Neutron Shield

cf64 51 \$ Flagging Cells for leak-through,

fkyshine

fc74 $474: \mathrm{P}$ 45oton Dose behind Neutron Shield, Concrete floor

f74:p $45 \quad$ Operator behind Neutron Shield

fc84 Photon Dose behind Neutron shield, Sum of All Cells FLAGGing!!

$\begin{array}{ll}\text { fc84 } & \text { Photon Dose behind Neutron Shield, Sum of All Cells } \\ \text { f84:p } 45 & \$ \text { Operator behind Neutron Shield }\end{array}$

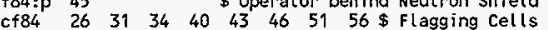

fc5 Photon dose at $30 \mathrm{~cm}$ from outside surface of Calciner

$\begin{array}{llll}c & T o t a l & 0 \text { ist from outside }=30.0+29.8450+40.0050=69.85 \quad \mathrm{~cm}\end{array}$

fc15 Photon dose at $30 \mathrm{~cm}$ from outside surface of Glovebox

c Total Dist from outside $=30.0+83.5025=113.5025 \mathrm{~cm}$

$f 15: p \quad-168.5925 \quad 113.5025 \quad 125.9713 \quad 0$

fc25 Photon dose at 5 feet from outside surface of Glovebox

c Total Dist from outside $=152.8+83.5025=235.9025 \mathrm{~cm}$

$f 25: p \quad-168.5925235 .9025125 .9713 \quad 0$

fc35 Photon dose at 6 in in front of lower $n$ shield

Total Dist from outside $=191.4525-6^{* 2.54}=176.2125 \mathrm{~cm}$

f35:P $0176.2125 \quad 91.44 \quad 0$

fc45 Photon dose in middle of outer $1 / 8^{11}$ SST $n$ shield

c Total Dist from outside $=(191.7700+192.0875) / 2=191.9288 \mathrm{~cm}$

f45:P $0191.928891 .44 \quad 0$

fc55 Photon dose in middle of 5.0 " lower $n$ shield

c Total Dist from outside $=(192.0875+204.7875) / 2=198.4375 \mathrm{~cm}$ 
f55:p $\quad 0198.4375 \quad 91.44 \quad 0$

fc65 Photon close in middle of inner $1 / 8^{11}$ stainless steel $n$ shield c Total Dist from outside $=(204.7875+205.1050) / 2=204.9462 \mathrm{~cm}$ f65:p $0204.9462 \quad 91.44 \quad 0$

fc75 Photon dose at 6 in. behind inside lower $n$ shield

c Total Dist from outside $=205.4225+6 * 2.54=220.6225 \mathrm{~cm}$

f75:P $\quad 0220.6625 \quad 91.44 \quad 0$

fC85 Photon dose at 6 in in tront of upper $n$ shield

c Total from outside $=191.4525-6 * 2.54=176.2125 \mathrm{~cm}$

f85:P 0176.2125182 .88 o

$f c 95$ Photon dose in middle of outer $3 / 4^{\prime \prime}$ glass $n$ Shield

c Total Dist from outside $=(191.4525+193.3575) / 2=192.4050 \mathrm{~cm}$ f95:P $0192.4050 \quad 182.88 \quad 0$

fc105 Photon dose in middle of 4.0" upper $n$ Shield

c Total Dist from outside $=(193.3575+203.5175) / 2=198.4375 \mathrm{~cm}$ f105:p $0198.4375 \quad 182.88 \quad 0$

fc115 Photon dose in middle of inner 3/4" glass $n$ Shield

c Total Dist from outside $=(203.5175+205.4225) / 2=204.4700 \mathrm{~cm}$ f115:P $0204.4700 \quad 182.88 \quad 0$

fc125 photon dose at 6 in behind inside upper $n$ shield

Iotal ist from outside $=205.4225+6 \star 2.54=220.6225 \mathrm{~cm}$

f125:p $0220.6625 \quad 182.88 \quad 0$

print $10 \quad 110 \quad 120$

ctme 180.0
CFTOBANP. INP Feed Jank, $3.5 \mathrm{~kg} \mathrm{Pu}$, neutron and capture gammas

NEUTRON DOSe Rate Calcs for calciner - CFTOBANP

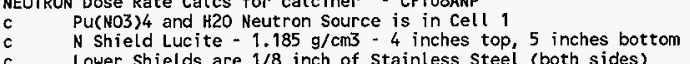

Lower Shields are $1 / 8$ inch of Stainless Steel (both sides)
Upper shields are $3 / 4$ inch of Glass (no Boron)(both sides)

Upper Shields are $3 / 4$ inch of Glass (no Boron) Moth sides)

c Full Room Model with Concrete Walls, Roof,

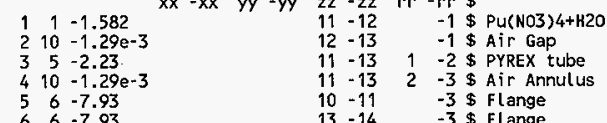

7 10 $-1.930-3 \quad 24-25 \quad 34-359-15 \quad(-10: 14: 3)$ \$ Inside Glovebox

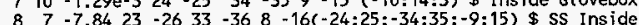

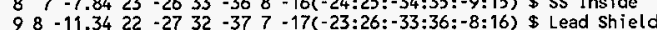

$10 \quad 7-7.8421 \quad-28 \quad 31-386-18(-22: 27:-32: 37:-7: 17)$ \$ S Outside

$11 \quad 10-1.29 \mathrm{e}-3 \quad 21-28 \quad 31-38 \quad 5-6 \quad 5 \quad$ S Under Glovebox

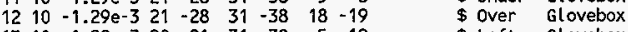

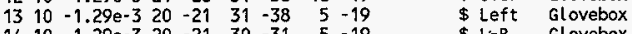

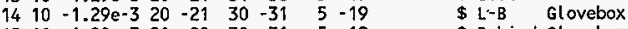

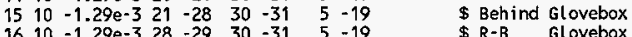

$1710-1,290-328-29-31-38$

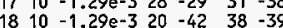
19 10 $-1.29 \mathrm{e}-3 \quad 20 \quad-42 \quad 38-39$

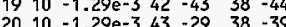

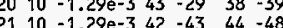

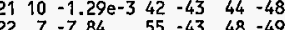
$\begin{array}{lllll}-7.84 & 55 & -43 & 48 & -49\end{array}$ $\begin{array}{lllllll}23 & 14 & -1.185 & 52 & -43 & 49 & -50\end{array}$ $\begin{array}{lllllll}24 & 7 & -7.84 & 53 & -43 & 50 & -51\end{array}$ $25 \quad 10-1.29 e-3 \quad 56-43 \quad 51-47$

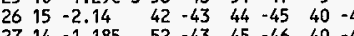

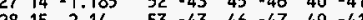
$\begin{array}{lll} & -1\end{array}$

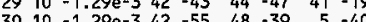

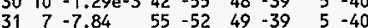

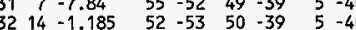
$\begin{array}{lllllllll}33 & 7 & -7.84 & 53 & -56 & 51 & -39 & 5 & -40\end{array}$

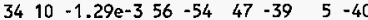

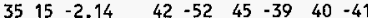

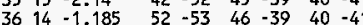

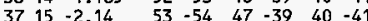

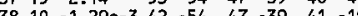

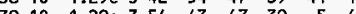

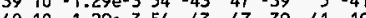
40 10 -1.29e-3 $54-43 \quad 47-39-41-19$ $41 \quad 10-1.29 e-3 \quad 60-20 \quad 30-39 \quad 5-19$ $4210-1.29 e-3 \quad 60-20 \quad 63-30 \quad 5-19$

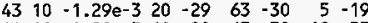

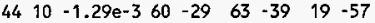

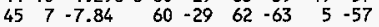
$\begin{array}{lll}46 \quad 7 & -7.84\end{array}$ $4711-2.30$ $4811-2.30$ $4911-2.30$ $\begin{array}{lll}49 & 11 & -2.30 \\ 50 & 11 & -2.30\end{array}$ 510 $\begin{array}{llll}60 & -29 & 39 & -6\end{array}$ $\begin{array}{llll}59 & -60 & 62 & -64\end{array}$ $\begin{array}{ll}5 & -57 \\ 5 & -57\end{array}$ $\begin{array}{llllll}59 & -61 & 62 & -64 & 57 & -58\end{array}$ $59-61 \quad 62-64 \quad 4 \quad-5$ $61:-59: 64:-62:-4: 58$
Right Glovebox Front Glovebox $\$$ Front Glovebox Front Glovebox \$ Bot Fr Outside air Bot Fr Outer 1/8' Bot Fr Middle $5.0 "$ Bot fr Inside 1/8" Bot fr minside 1/8"

- Bot fr Inside air

Top Fr Outer 3/4"

Top Fr Midale 4.0"

Top Fr Inside 3/4"

Above Shield

Bot L Outside ai

\$ot 1 Outer $1 / 8$

$\$$ Bot 1 Middle $1.0^{\prime \prime}$

Bot 1 Inside $1 / 8^{\prime \prime}$

Bot 1 Inside air

$\$$ TOP L Outer 3/4"

$\$$ Top L Middle 1.0"

\$ Top L Inside 3/4"

$\$$ Above Shield

Behind $n$ Shield

\$ Behind n shield

$\$$ Room, Left side

$\$$ Room, Left Back

Room, Back Part

$\$$ Room to Ceiling

$\$$ SS Wall Back

ss Hall Front

$\$$ Concrete Wall Left

- Concrete Wall Right

$\$$ Concrete Roof

Concrete Floor

OUTSIDE WORLD

$\begin{array}{lr}\text { cz } & 7.7610 \\ \text { cz } & 8.4534 \\ \text { cz } & 14.2875 \\ \text { pz } & -60.9600 \\ \text { pz } & 0.0000 \\ \text { pz } & 86.3600 \\ \text { pz } & 86.5981 \\ \text { pz } & 87.0744 \\ \text { pz } & 87.3125 \\ \text { pz } & 171.4500 \\ \text { pz } & 171.7200 \\ \text { pz } & 224.5663 \\ \text { pz } & 233.6800 \\ \text { pz } & 234.9500 \\ \text { pz } & 265.7475 \\ \text { pz } & 265.9856 \\ \text { pz } & 266.4619 \\ \text { pz } & 266.7000\end{array}$

$\$$ FEED TANK O.R. - (5/16"1-0.040:1)

FEED TANK O.R.

FEED TANK FLANGE O.R.

$\$$ CONCRETE FLOOR bottom (24" thick)

$\$$ CONCRETE FLOOR surface

$\$$ GLOVEBOX bottom surface

\$ GLOVEBOX Stainless steel (3/32")

$\$$ GLOVEBOX Lead Shield (3/16"i)

\$ GLOVEBOX Stainless steel (3/32ti)

FEED TANK Bottom Flange bottom

FEED TANK Bottom Flange top

$\$$ FEED TANK Bot on Flange top

$\$$ FEED IANK TOP Flange bot tom

$\$$ FEED TANK TOP Flange top

$\$$ GLOVEBOX top inside surface

\$ GLOVEBOX stainless steel (3/32")

$\$$ GLOVEBOX Lead Shield (3/16")

\$ GLOVEBOX stainless steel (3/32") 


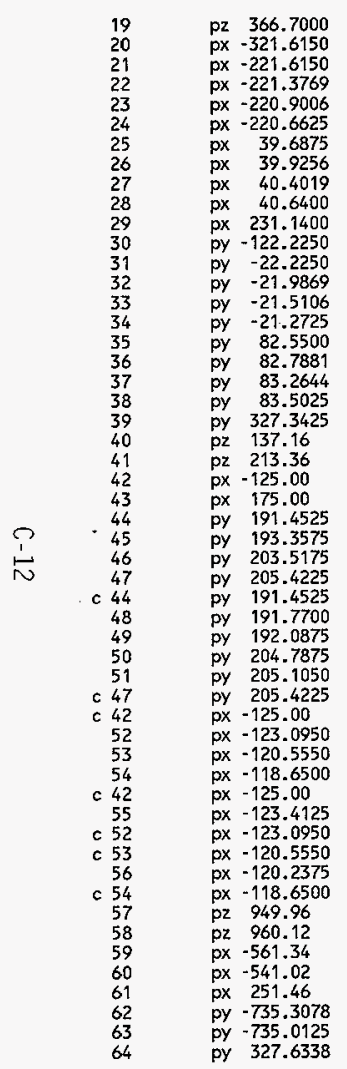

$\$$ MODEL TOP (GB roof $+100 \mathrm{~cm}$ )

$\$$ MODEL LEFT SIDE (GB side $+100 \mathrm{~cm}$ )

$\$$ GLOVEBOX left surface

\$ GLOVEBOX Stainless Steel (3/32'")

$\$$ GLOVEBOX Lead shield (3/16/1)

$\$$ GLOVEBOX Stainless Steel (3/32")

$\$$ GLOVEBOX right inside surface

\$ GLOVEBOX stainless steel (3/32")

$\$$ GLOVEBOX Lead shield (3/16"1)

\$ GLOVEBOX Stainless steel (3/32")

$\$$ MODEL RIGHT SIDE (GB side $+75^{\prime \prime}$ )

$\$$ MODEL BACK SIDE (GB back $+100 \mathrm{~cm}$ )

$\$$ GLOVEBOX back surface

\$ GLOVEBOX Stainless Steel (3/32")

$\$$ GLOVEBOX Lead Shield (3/16"1)

\$ GLOVEBOX stainless steel (3/32")

$\$$ GLOVEBOX front inside surface

\$ GLOVEBOX Stainless Steel (3/32")

\$ GLOVEBOX Lead Shield (3/16")

\$ GLOVFBOX Stainless Steel (3/32")

$\$$ MODEL FRONT SIDE (GB front +96")

$\$ 4.5$ ' High $n$ Shield

$\$+2.5$ : Top $n$ shield

$\$$ Left End $n$ Shield

$\$$ Right End $n$ Shield

$\$ 3 / 4 "$ n Shield Material outside

$\$ 4.0 "$ n Shield Material outside

$\$ 3 / 4 "$ n Shield Material outside

$\$$ Operator side $n$ Shield

$\$ 3 / 4^{\prime \prime} n$ Shield Material outside

$\$ 1 / 8^{\prime \prime}$ n Shield Material outside

$\$ 5$.0" n Shield Material outside

$\$ 1 / 8$ " n shield Material outside

$\$ 1 / 8$ " n Shield Material outside

$\$$ Operator side $n$ Shield

$\$ 3 / 4^{: 11} n$ Shield Material outside

$\$ 1.0^{\prime \prime}$ n Shield Material outside

$\$$ Operator side $n$ shield

\$3/4" n Shield Material outside

\$1/8" n Shield Material outside

$\$ 1.0 "$ n shield Material outside

$\$ 3 / 4 "$ n shield Material outside

1/8" n shield Material inside

$\$ 1 / 8$ n

Operator side $n$ Shield

$\$$ Ceiling Surface [H-2-16174]
$\$ 4$ inch concrete roof [H-2-16174]

$\$ 4$ inch concrete roof [H-2-16174]

$\$ 8$ inch concrete wall left side

\$ Left Wall Surface [H-2-16140]

$\$ 8$ inch concrete wall right side

$\$$ Back Wall Surface [H-2-16140]

$\$ 18$ Gauge Metal $\left(.0516^{11 *}(4.0 / 1.8) \Rightarrow .2913 \mathrm{~cm}\right)$

mode $\quad \mathrm{n}$ p

imp:p $149 r^{\circ}$

$c$ wgt $=3500 * 105=367500$ Low $240 \mathrm{Pu}$

wgt $=3500 * 215=752500 \mathrm{Mid} 240 \mathrm{Pu}$

c wgt $=3500 * 295=1032500 \mathrm{High} 240 \mathrm{Pu}$

sdef cel $=1 \quad$ erg $=d 2 \quad$ pos $=0000$ rad $=d 3$

cel $=1 \quad$ erg $=d 2 \quad$ pos $=0 \begin{array}{llll}0 & 0 & 0 & \mathrm{rad}=\mathrm{d} 3\end{array}$

$\mathrm{sc2}$ (alpha, $n$ ) and fission spectrum

$\begin{array}{llll}\mathrm{si2} & \mathrm{s} & 35 & 36 \\ \mathrm{sp2} & & 0.504 & 0.496\end{array}$

$\mathrm{sc3}$ radius

si3 7.7610

$\mathrm{sp3}-211$

sc4 extent

si4 $171.7200 \quad 224.5663$

sp4 -210

sc35 (alpha, n) neutron source on OXYGEN from Jacom and Liskien

c Energy of the alpha particles $=5.5 \mathrm{MeV}$

si35 of the alph

$\begin{array}{ll}\text { si35 } & \text { sp35 } \\ 0.0 & 0.00 \\ 0.1 & 0.68 \\ 0.2 & 0.76 \\ 0.3 & 0.74 \\ 0.4 & 0.88 \\ 0.5 & 1.20 \\ 0.6 & 1.35 \\ 0.7 & 1.48 \\ 0.8 & 1.62 \\ 0.9 & 1.55 \\ 1.0 & 1.28 \\ 1.1 & 1.34 \\ 1.2 & 1.45 \\ 1.3 & 1.63 \\ 1.4 & 1.76 \\ 1.5 & 1.80 \\ 1.6 & 1.89 \\ 1.7 & 2.05 \\ 1.8 & 2.28 \\ 1.9 & 2.37 \\ 2.0 & 2.49 \\ 2.1 & 2.50 \\ 2.2 & 2.61 \\ 2.3 & 2.65 \\ 2.4 & 2.54 \\ 2.5 & 2.43 \\ 2.6 & 2.34 \\ 2.7 & 2.73 \\ 2.8 & 2.06 \\ 2.9 & 2.00 \\ 3.0 & 1.88 \\ 3.1 & 1.66 \\ 3.2 & 1.56 \\ 3.3 & 1.34 \\ & \end{array}$




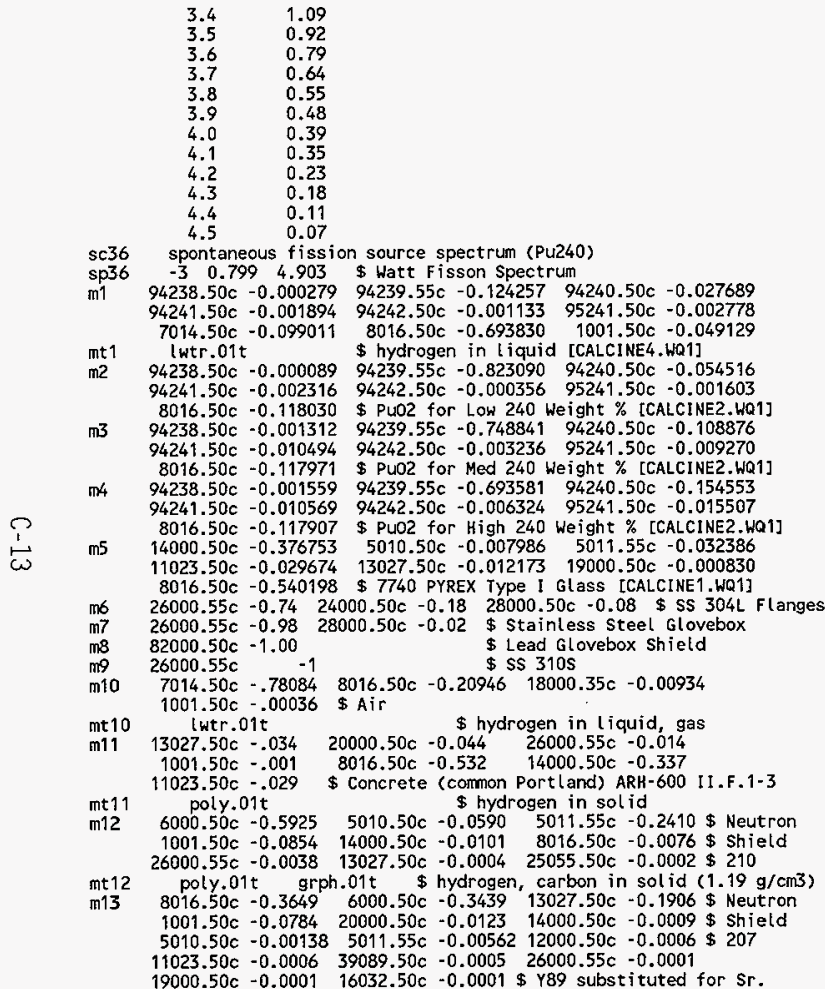

$11023.50 c-0.030923 \quad 13027.50 c-0.01268519000 .50 c-0.000865$ $8016.50 \mathrm{c}-0.562924 \$ 7740$ PYREX TYPe 1 GLASS [CALCINE1.WQ1] m16 6000.50c-0.755751 1001.50c-0.055491 8016.50c -0.188758 \$ Lexan me16 poly.01t grph.01t \$ hydrogen in solid $(1.2 \mathrm{~g} / \mathrm{cm} 3)$ Lexan c. Photon dose conversion factors from ANS 6.1.1 1991

de4 $\begin{array}{lllllll}\log & .01 & .015 & .02 & .03 & .04 & .05\end{array}$

$\begin{array}{llllll}.01 & .015 & .02 & .03 & .04 & .05 \\ .06 & .08 & .10 & .15 & .20 & .30\end{array}$

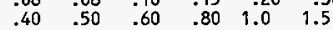

$\begin{array}{llllll}2.0 & 3.0 & 4.0 & 5.0 & 6.0 & 8.0\end{array}$

df4 $\log 2.232 \mathrm{e}-5 \quad 5.652 \mathrm{e}-5$ 8.568e-5 $1.184 \mathrm{e}-41.314 \mathrm{e}-41.382 \mathrm{e}-4$

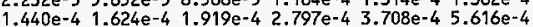
$7.416 \mathrm{e}-4 \quad 9.144 \mathrm{e}-4 \quad 1.076 \mathrm{e}-3 \quad 1.379 \mathrm{e}-3 \quad 1.656 \mathrm{e}-3 \quad 2.246 \mathrm{e}-3$ $2.758 \mathrm{e}-3 \quad 3.672 \mathrm{e}-3 \quad 4.500 \mathrm{e}-3 \quad 5.292 \mathrm{e}-3 \quad 6.012 \mathrm{e}-3 \quad 7.488 \mathrm{e}-3$ $8.892 \mathrm{e}-3 \quad 1.040 \mathrm{e}-2$

fc4 Photon Dose behind Neutron Shield, Right Side Leak Around

$\mathrm{f} 4: \mathrm{p} \quad 39 \quad$ \$ Operator behind Neutron Shield

cf 20 \$ Flagging Cells for leak-through, cturine

Photon dose conversion factors from ANS 6.1.1 1991

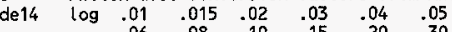

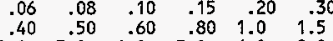

$\begin{array}{llllll}2.0 & 3.0 & 4.0 & 5.0 & 6.0 & 8.0\end{array}$

df $14 \quad \log 2.232 \mathrm{e}-5 \quad 5.652 \mathrm{e}-5 \quad 8.568 \mathrm{e}-5 \quad 1.184 \mathrm{e}-4 \quad 1.314 \mathrm{e}-4 \quad 1.382 \mathrm{e}-4$ $1.440 \mathrm{e}-4 \quad 1.624 \mathrm{e}-4 \quad 1.919 \mathrm{e}-4 \quad 2.797 \mathrm{e}-4 \quad 3.708 \mathrm{e}-4 \quad 5.616 \mathrm{e}-4$

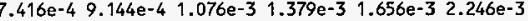
$2.758 \mathrm{e}-3 \quad 3.672 \mathrm{e}-3 \quad 4.500 \mathrm{e}-3 \quad 5.292 \mathrm{e}-3 \quad 6.012 \mathrm{e}-3 \quad 7.488 \mathrm{e}-3$ $8.892 \mathrm{e}-3 \quad 1.040 \mathrm{e}-2$

fc14 Photon Dose behind Neutron Shield, Front Lower $n$ Shield Leak Through f14:p 39 \$ Operator behind Neutron Shield

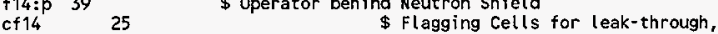
skyshine

Photon dose conversion factors from ANS 6.1.1 1991

de24 $\log \begin{array}{llllll}.01 & .015 & .02 & .03 & .04 & .05\end{array}$

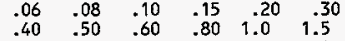

$\begin{array}{cccccc}2.0 & 3.0 & 4.0 & 5.0 & 6.0 & 8.0\end{array}$

df24 $\log 2.232 \mathrm{e}-5 \quad 5.652 \mathrm{e}-5 \quad 8.568 \mathrm{e}-5 \quad 1.184 \mathrm{e}-4 \quad 1.314 \mathrm{e}-4 \quad 1.382 \mathrm{e}-4$

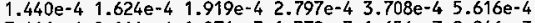

$\begin{array}{llllll}7.416 e-4 & 9.144 e-4 & 1.076 e-3 & 1.379 e-3 & 1.656 e-3 & 2.246 e-3\end{array}$

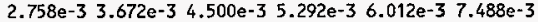

\section{$8.892 \mathrm{e}-31.040 \mathrm{e}-2$}

fc24 Photon Dose behind Neutron Shield, Front Upper $\mathrm{n}$ Shield Leak Through $\begin{array}{ll}\text { fc24 Photon Dose behind Neutron Shield, Front Upper } \\ \text { f24:p } 39 & \text { \$Operator behind Neutron Shield }\end{array}$
cf24:p 39
28
$\$$ Flagging Cells for leak-through,

$19000.50 \mathrm{c}-0.0001 \quad 16032.50 \mathrm{c}-0.0001 \$ \mathrm{Y} 89$ substituted for $\mathrm{Sr}$.

c Photon dose conversion factors from ANS 6.1.1 1991

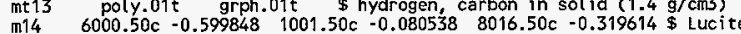

mt 14 poly.01t grph.01t \$hydrogen in solid $(1.185 \mathrm{~g} / \mathrm{cm} 3)$ Lucite

m15 $14000.50 \mathrm{c}-0.392603 \$ 7740$ PYREX Type I Glass [no Boron, $2.14 \mathrm{~g} / \mathrm{cm} 3$ ]

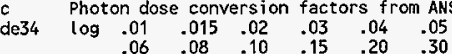

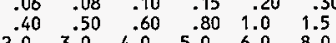


df 34

$10.0 \quad 12.0$

$\log 2.232 e-5 \quad 5.652 e-5 \quad 8.568 e-5 \quad 1.184 e-4 \quad 1.314 e-4 \quad 1.382 e-4$ $1.440 \mathrm{e}-4 \quad 1.624 \mathrm{e}-4 \quad 1.919 \mathrm{e}-4 \quad 2.797 \mathrm{e}-4 \quad 3.708 \mathrm{e}-4 \quad 5.616 \mathrm{e}-4$

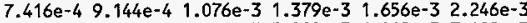
$2.758 \mathrm{e}-3 \quad 3.672 \mathrm{e}-3 \quad 4.500 \mathrm{e}-3 \quad 5.292 \mathrm{e}-3 \quad 6.012 \mathrm{e}-3 \quad 7.488 \mathrm{e}-3$ $8.892 \mathrm{e}-3 \quad 1.040 \mathrm{e}-2$

fc34 Photon Dose behind Neutron Shield, Left Lower $n$ Shield Leak Through $\begin{array}{lll}\mathrm{f} 34: \mathrm{p} & 39 & \text { \$ Operator behind Neutron Shield } \\ \mathrm{cf} 34 & 34 & \text { \$Flagging Cells for leak-through, }\end{array}$ c Photon dose conversion factors from ANS 6.1.1 1991

de44 $\log \begin{array}{llllll}.01 & .015 & .02 & .03 & .04 & .05\end{array}$

$\begin{array}{cccccc}.06 & .08 & .10 & .15 & .20 & .30 \\ .40 & .50 & .60 & .80 & 1.0 & 1.5 \\ 2.0 & 3.0 & 4.0 & 5.0 & 6.0 & 8.0 \\ 10.0 & 12.0 & & & & \end{array}$

df44

$$
10.0 \quad 12.0
$$

2.232e-5 $5.652 e-5 \quad 8.568 e-5 \quad 1.184 e-4 \quad 1.314 e-4 \quad 1.382 e-4$ $1.440 \mathrm{e}-4 \quad 1.624 \mathrm{e}-4 \quad 1.919 \mathrm{e}-4 \quad 2.797 \mathrm{e}-4 \quad 3.708 \mathrm{e}-4 \quad 5.616 \mathrm{e}-4$

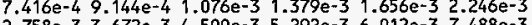
$2.758 \mathrm{e}-3 \quad 3.672 \mathrm{e}-3$ 4.500e-3 $5.292 \mathrm{e}-3$ $6.012 \mathrm{e}-3 \quad 7.488 \mathrm{e}-3$ $8.892 e-31.040 e-2$

fc44 Photon Dose behind Neutron Shield, Left Upper $n$ Shield Leak Through df84 f44:p 39 \$ Operator behind Neutron Shield

cf44 37 \$ Flagging Cells for leak-through, skyshine

c Photon dose conversion factors from ANS 6.1.1 1991

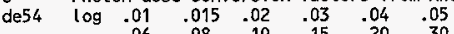

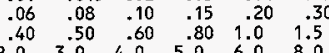

$$
\begin{array}{cccccc}
.40 & .50 & .60 & .80 & 1.0 & 1.5 \\
2.0 & 3.0 & 4.0 & 5.0 & 6.0 & 8.0
\end{array}
$$

df54 $\log 2.232 e-5 \quad 5.652 e-5 \quad 8.568 e-5 \quad 1.184 e-4 \quad 1.314 e-4 \quad 1.382 e-4$

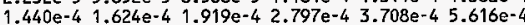

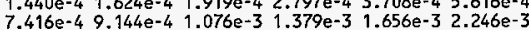

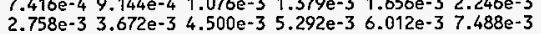
$\begin{array}{ll}2.758 \mathrm{e}-3 & 3.672 \mathrm{e}-3 \\ 8.892 \mathrm{e}-3 & 1.040 \mathrm{e}-2\end{array}$

fc54 Photon Dose behind Neutron Shield, Roof Reflection

f54:p 39 \$ Operator behind Neutron Shield

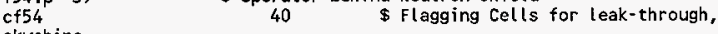

skyshin

c Photon dose conversion factors from ANS 6.1.1 1991

de64 $\begin{array}{lllllll}\log & .01 & .015 & .02 & .03 & .04 & .05\end{array}$

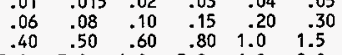

$\begin{array}{llllll}2.0 & 3.0 & 4.0 & 5.0 & 6.0 & 8.0\end{array}$

$10.0 \quad 12.0$

$\log 2.232 \mathrm{e}-5 \quad 5.652 \mathrm{e}-5 \quad 8.568 \mathrm{e}-5 \quad 1.184 \mathrm{e}-4 \quad 1.314 \mathrm{e}-4 \quad 1.382 \mathrm{e}-4$

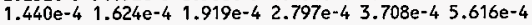

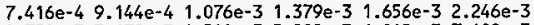
$2.758 \mathrm{e}-3 \quad 3.672 \mathrm{e}-3 \quad 4.500 \mathrm{e}-3 \quad 5.292 \mathrm{e}-3 \quad 6.012 \mathrm{e}-3 \quad 7.488 \mathrm{e}-3$ $8.892 \mathrm{e}-3 \quad 1.040 \mathrm{e}-2$

fc64 Photon Dose behind Neutron Shield, Steel Wall Back Reflection

$\begin{array}{lll}\text { f64:p } 39 & 39 & \$ \text { Operator behind Neutron Shield } \\ \text { cf64 } & 45 \quad \text { \$ Flagging Cells for leak-through, }\end{array}$

skyshine

c Photon dose conversion factors from ANS 6.1.1 1991

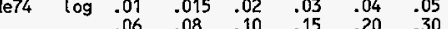

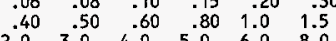

$\begin{array}{llllll}2.0 & 3.0 & 4.0 & 5.0 & 6.0 & 8.0\end{array}$

df74 $\log 2.232 \mathrm{e}-5 \quad 5.652 \mathrm{e}-5 \quad 8.568 \mathrm{e}-5 \quad 1.184 \mathrm{e}-4 \quad 1.314 \mathrm{e}-4 \quad 1.382 \mathrm{e}-4$

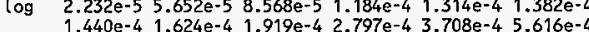

$\begin{array}{llllll}1.440 \mathrm{e}-4 & 1.624 \mathrm{e}-4 & 1.919 \mathrm{e}-4 & 2.797 \mathrm{e}-4 & 3.708 \mathrm{e}-4 & 5.616 \mathrm{e}-4 \\ 7.416 \mathrm{e}-4 & 9.144 \mathrm{e}-4 & 1.076 \mathrm{e}-3 & 1.379 \mathrm{e}-3 & 1.656 \mathrm{e}-3 & 2.246 \mathrm{e}-3\end{array}$

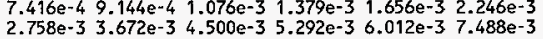
$8.892 \mathrm{e}-3$ 1.040e-2

fc74 Photon Dose behind Neutron Shield, Concrete Floor Effect

f74:p 39 \$ Operator behind Neutron Shield

c Photon dose conversion factors from ANS 6.1 .11991

$\begin{array}{lllllll}\text { de84 } \log & .01 & .015 & .02 & .03 & .04 & .05\end{array}$

$\begin{array}{llllll}.06 & .08 & .10 & .15 & .20 & .30 \\ .40 & .50 & .60 & .80 & 1.0 & 1.5\end{array}$

$\begin{array}{rrrrrr}.40 & .50 & .60 & .80 & 1.0 & 1.5 \\ 2.0 & 3.0 & 4.0 & 5.0 & 6.0 & 8.0\end{array}$

$10.0 \quad 12.0$

$\log 2.232 \mathrm{e}-5 \quad 5.652 \mathrm{e}-5 \quad 8.568 \mathrm{e}-5 \quad 1.184 \mathrm{e}-4 \quad 1.314 \mathrm{e}-4 \quad 1.382 \mathrm{e}-4$

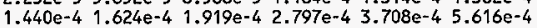
$7.416 \mathrm{e}-4 \quad 9.144 \mathrm{e}-4 \quad 1.076 \mathrm{e}-3 \quad 1.379 \mathrm{e}-3 \quad 1.656 \mathrm{e}-3 \quad 2.246 \mathrm{e}-3$ $2.758 \mathrm{e}-3 \quad 3.672 \mathrm{e}-3 \quad 4.500 \mathrm{e}-3 \quad 5.292 \mathrm{e}-3 \quad 6.012 \mathrm{e}-3 \quad 7.488 \mathrm{e}-3$ $8.892 \mathrm{e}-31.040 \mathrm{e}-2$

fc84 Photon Dose behind Neutron Shield, Sum of All Cells FLAGgING! f84:p $39 \quad$ \$ Operator behind Neutron Shield

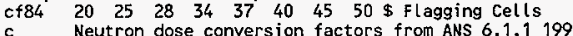

$\begin{array}{lllllll}\text { de } 0 & 2.50 \mathrm{e}-08 & 1.00 \mathrm{e}-07 & 1.00 \mathrm{e}-06 & 1.00 \mathrm{e}-05 & 1.00 \mathrm{e}-04 & 1.00 \mathrm{e}-03\end{array}$

$1.00 \mathrm{e}-02 \quad 2.00 \mathrm{e}-02 \quad 5.00 \mathrm{e}-02 \quad 1.00 \mathrm{e}-01 \quad 2.00 \mathrm{e}-01 \quad 5.00 \mathrm{e}-01$

$1.00 \mathrm{e}+00 \quad 1.50 \mathrm{e}+00 \quad 2.00 \mathrm{e}+00 \quad 3.00 \mathrm{e}+00 \quad 4.00 \mathrm{e}+00 \quad 5.00 \mathrm{e}+00$

$1.00 \mathrm{e}+0031.50 \mathrm{e}+00 \quad 2.00 \mathrm{e}+00 \quad 3.00 \mathrm{e}+00 \quad 4.00 \mathrm{e}+00$

dfo $\quad 1.440 \mathrm{e}-03 \quad 1.584 \mathrm{e}-03 \quad 1.735 \mathrm{e}-03 \quad 1.606 \mathrm{e}-03 \quad 1.490 \mathrm{e}-03 \quad 1.379 \mathrm{e}-03$

$\begin{array}{llllll}1.440 \mathrm{e}-03 & 1.584 \mathrm{e}-03 & 1.735 \mathrm{e}-03 & 1.606 \mathrm{e}-03 & 1.490 \mathrm{e}-03 & 1.379 \mathrm{e}-03 \\ 1.631 \mathrm{e}-03 & 2.113 \mathrm{e}-03 & 3.924 \mathrm{e}-03 & 7.128 \mathrm{e}-03 & 1.390 \mathrm{e}-02 & 3.132 \mathrm{e}-02\end{array}$

$\begin{array}{llllll}1.631 \mathrm{e}-03 & 2.113 \mathrm{e}-03 & 3.924 \mathrm{e}-03 & 7.128 \mathrm{e}-03 & 1.390 \mathrm{e}-02 & 3.132 \mathrm{e}-02 \\ 5.148 \mathrm{e}-02 & 6.588 \mathrm{e}-02 & 7.704 \mathrm{e}-02 & 9.504 \mathrm{e}-02 & 1.080 \mathrm{e}-01 & 1.177 \mathrm{e}-01\end{array}$

$\begin{array}{lllll}5.148 \mathrm{e}-02 & 6.588 \mathrm{e}-02 & 7.704 \mathrm{e}-02 & 9.504 \mathrm{e}-02 & 1.080 \mathrm{e}-01 \\ 1.249 \mathrm{e}-01 & 1.314 \mathrm{e}-01 & 1.368 \mathrm{e}-01 & 1.476 \mathrm{e}-01 & 1.728 \mathrm{e}-01\end{array}$

fc204 Neutron Dose behind Neutron Shield, Right Side Leak Around

$4204: \mathrm{n} 39 \quad \$$ Operator behind Neutron Shield

$4 \mathrm{~m} 204 \quad 2.0 \quad$ \$ Factor of 2 times the ANS 6.1 .11991 Standard.

cf 20420

skyshine

fc214 Neutron Dose behind Neutron Shield, front Lower n Shield Leak

Through

f $214: \mathrm{n} 39 \quad \$$ Operator behind Neutron Shield

fm214 2.0 \$ Factor of 2 times the ANS 6.1.1 1991 standard.

cf214 25

skyshine

Through

$\mathrm{f} 224: \mathrm{n} 39$

\$Operator behind Neutron Shield

\$ Factor of 2 times the ANS 6.1 .11991 standard. $\$$ Flagging cells for leak-through

$\{224$

28

\$ Flagging Cells for leak-through, 
skyshine

fc234 Neutron Dose behind Neutron Shield, Left Lower $n$ Shield Leak Through

f234:n 39

$\begin{array}{ll}f 234: n & 39 \\ f m 234 & 2.0\end{array}$

fm234

$\$$ Operator behind Neutron Shield

\$ Factor of 2 times the ANS 6.1 .11991 Standard.

cf234

$\$$ Flagging Cells for leak-through,

fc244 Neutron Dose behind Neutron Shield, Left Upper $n$ Shield Leak

Through

$\begin{array}{ll}\text { f244:n } 39 & \text { \$ Operator behind Neutron Shield } \\ \text { fm244 2.0 } & \text { Factor of } 2 \text { times the ANS } 6.1 .1 \text { 1991 Standard. }\end{array}$

cf244

skyshine

fc254 Neutron Dose behind Neutron Shield, Roof Reflection

f254:n 39 \$ Operator behind Neutron Shield

fm254 2.0 Factor of 2 times the ANS 6.1 .11991 standard.

40 Flagging Cells for leak-through,

skyshine

fc264 Neutron Dose behind Neutron Shield, Steel Wall Back Reflection
f264:n 39

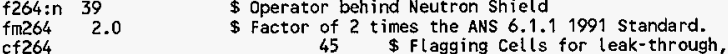

cf264

skyshine

fc274 Neutron Dose behind Neutron Shield, Concrete Floor Effect

f274:n 39 \& Operator behind Neutron Shield

fm274 $2.0 \quad$ \$ Factor of 2 times the ANS 6.1 .11991 standard.

() 1274

50 \& Flagging Cellis for leak-through

skyshine

fc284 Neutron Dose behind Neutron Shield, Sum of All Cells FLAGGING!!

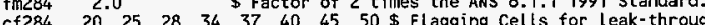

$\mathrm{fc5}$ Neutron dose at $30 \mathrm{~cm}$ from outside surface of $6 \mathrm{in}$. pipe

Total Dist from outside $=30.0+8.4534=38.4534 \mathrm{~cm}$

$\mathrm{f} 5 \mathrm{n} \quad \mathrm{0} \quad 38.4534200 .0$

fm5 2.0 \$ Factor of 2 times the ANS 6.1 .11991 standard.

fc15 Neutron dose at $30 \mathrm{~cm}$ from outside surface of Glovebox

c Total Dist from outside $=30.0+83.5025=113.5025 \mathrm{~cm}$

$+15: n \quad 0 \quad 113.5025200 .0 \quad 0$

fm15 2.0 \$ Factor of 2 times the ANS 6.1 .11991 standard.

fc25 Neutron dose at 5 feet from outside surface of Glovebox

c Total Dist from outside $=152.8+83.5025=235.9025 \mathrm{~cm}$

f25: $\mathrm{n} 0235.9025200 .0$

\$ Factor of 2 times the ANS 6.1.1 1991 standard.

fc35 Neutron dose at 6 in. in front of lower $n$ shield

c Total Dist from outside $=191.4525-6{ }^{\star} 2.54=176.2125 \mathrm{~cm}$

f $35: \mathrm{n} \quad 0176.2125 \quad 91.44 \quad 0$

fm35 2.0 \$ Factor of 2 times the ANS 6.1 .11991 standard.

fc45 Neutron dose in middle of outer $1 / 8^{\prime \prime}$ SST $n$ Shield

c Total Dist from outside $=(191.7700+192.0875) / 2=191.9288 \mathrm{~cm}$

f $45: n \quad 0191.9288 \quad 91.440$

fm45 2.0 \$ Factor of 2 times the ANS 6.1 .11991 Standard.

fc55 Neutron dose in middle of $5.0^{\prime \prime}$ lower $n$ shield c Total Dist from outside $=(192.0875+204.7875) / 2=198.4375 \mathrm{~cm}$ f55:n $\quad 0198.4375 \quad 91.44 \quad 0$

$f \mathrm{~m} 55 \quad 2.0$ \$ Factor of 2 times the ANS 6.1.1 1991 standard.

fc65 Neutron dose in middle of inner 1/8" Stainless steel $n$ shield

c Total Dist from outside $=(204.7875+205.1050) / 2=204.9462 \mathrm{~cm}$

f65:n $0204.9462 \quad 91.44 \quad 0$

fm65 2.0 \$ Factor of 2 times the ANS 6.1.1 1991 standard.

fc75 Neutron dose at 6 in. behind inside lower $n$ shield

Total Dist from outside $=205.4225+6 * 2.54=220.6225 \mathrm{~cm}$

f75:n $0220.6625 \quad 91.44 \quad 0$

2.0 \& Factor of 2 times the ANS 6.1 .11991 Standard. 2.0 Neutron dose at 6 in. in front of upper $n$ Shield
Totor of 2 times the AlS 6.1 .1

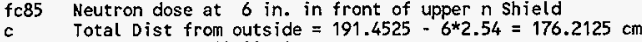
f85:n $0176.2125 \quad 182.88 \quad 0$

fmB5 2.0 \$ Factor of 2 times the ANS 6.1 .11991 standard.

fc95 Neutron dose in middle of outer 3/4" glass n Shield

c Total Dist from outside $=(191.4525+193.3575) / 2=192.4050 \mathrm{~cm}$ $0192.4050182 .88 \quad 0$

fm95 2.0 \$ Factor of 2 times the ANS 6.1.1 1991 standard.

feutron dose in middle of 4.0105 upper $n$ shield

c

c $105:$ Total Dist from outside $=(193.3575+203.5175) / 2=198.4375 \mathrm{~cm}$

2.0 Factor of 2 times the ANS 6.1 .11991 standard.

fc115 Neutron dose in middle of inner $3 / 4 "$ glass $n$ Shield

c Total Dist from outside $=(203.5175+205.4225) / 2=204.4700 \mathrm{~cm}$ f115:n 0204.4700182 .880

fm115 2.0 \$ Factor of 2 times the ANS 6.1 .11991 standard. fc125 Neutron dose at 6 in. behind inside upper $n$ Shield

c Total Dist from outside $=205.4225+6 * 2.54=220.6225 \mathrm{~cm}$

f125:n 0220.6625182 .88 o

fm125 $2.0 \quad \$$ Factor of 2 times the ANS 6.1 .11991 Standard.

print $10 \quad 110 \quad 120$

ctme 576.0

nps $1.6 \mathrm{e} 5$

CFTOBAP. IMP Feed Tank, $3.5 \mathrm{~kg} \mathrm{Pu}$, gamma ray calculation

PHOTON Dose Rate Calcs for calciner - CFTO8AP

c Pu(NO3)4 and H2O Photon Source is in Cell 1

N Shield Lucite - $1.185 \mathrm{~g} / \mathrm{cm} 3-4$ inches top, 5 inches bottom

Lower Shields are $1 / 8$ inch of Stainless Steel (both sides)

Upper Shields are $3 / 4$ inch of Glass (no Boron) (both sides)

Full Room Model with Concrete Walls, Roof, and Metal Partitions $x x-x x$ yy $-y y \quad z z-z z \quad r r-r r \$$

$1 \quad 1-1.582$

$\begin{array}{lrl}2 & 10 & -1.29 \mathrm{e}-3 \\ 3 & 5 & -2.23\end{array}$

$410-1.29 \mathrm{e}-3$

$\begin{array}{lll}5 & 6 & -7.93 \\ 6 & 6 & -7.93\end{array}$

$11-12$

$\begin{array}{ll}12 & -13 \\ 11 & -13\end{array}$

$\$$ Air Gap

$\begin{array}{llll}6 & 6 & -7.93\end{array}$

$11-13 \quad 2 \quad-3 \$$ Air Annulus

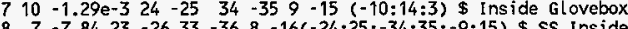

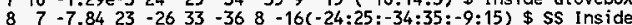


$98-11.3422-2732-377-17(-23: 26:-33: 36:-8: 16)$ \$ Lead Shield $107-7.8421 \quad-28 \quad 31-38 \quad 6-18(-22: 27:-32: 37:-7: 17)$ \$ Ss Outside

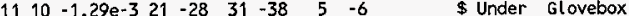

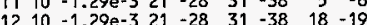

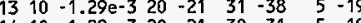
$14 \quad 10-1.29 \mathrm{e}-3 \quad 20-21 \quad 30-31 \quad 5-19$

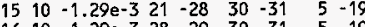
$16 \quad 10-1.29 e-3 \quad 28-29 \quad 30-31 \quad 5-19$

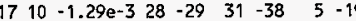

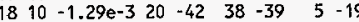

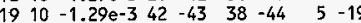

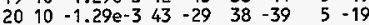

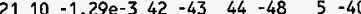

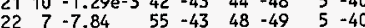

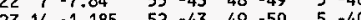

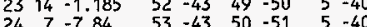

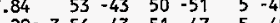
$25 \quad 10-1.29 \mathrm{e}-3 \quad 56-43 \quad 51-47 \quad 5-40$

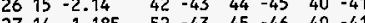
$\begin{array}{lllllllll}27 & 14 & -1.185 & 52 & -43 & 45 & -46 & 40 & -41\end{array}$ $\begin{array}{lllllllll}28 & 15 & -2.14 & 53 & -43 & 46 & -47 & 40 & -4\end{array}$

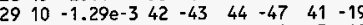

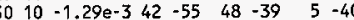
$\begin{array}{lllllllll}31 & 7 & -7.84 & 55 & -52 & 49 & -39 & 5 & -40\end{array}$

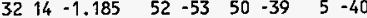
$\begin{array}{lllllllll}33 & 7 & -7.84 & 53 & -56 & 51 & -39 & 5 & -40\end{array}$

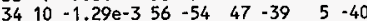
$\begin{array}{lllllllll}35 & 15 & -2.14 & 42 & -52 & 45 & -39 & 40 & -41\end{array}$

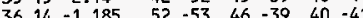
$\begin{array}{lllllllll}37 & 15 & -2.14 & 53 & -54 & 47 & -39 & 40 & -41\end{array}$

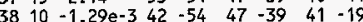

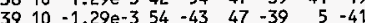

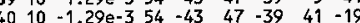

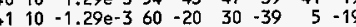

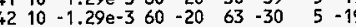

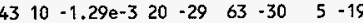

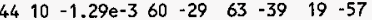
$\begin{array}{lllllllll}45 & 7 & -7.84 & 60 & -29 & 62 & -63 & 5 & -57\end{array}$ $\begin{array}{lllllllll}46 & 7 & -7.84 & 60 & -29 & 39 & -64 & 5 & -57\end{array}$ $\begin{array}{lllllllll}47 & 11 & -2.30 & 59 & -60 & 62 & -64 & 5 & -57\end{array}$ $\begin{array}{lllllllll}48 & 11 & -2.30 & 29 & -61 & 62 & -64 & 5 & -57\end{array}$

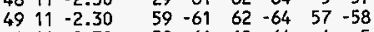

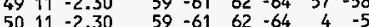
510

$61:-59: 64:-62:-4: 58$

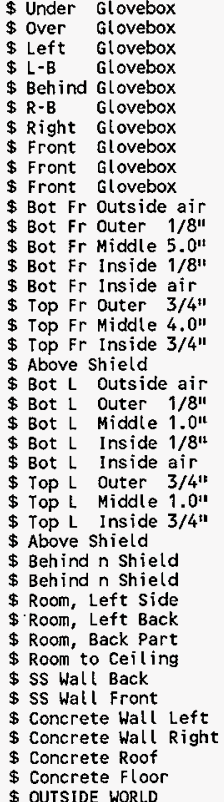

OUTSIDE WORLD

$\begin{array}{rrr}1 & \mathrm{cz} & 7.7610 \\ 2 & \mathrm{cz} & 8.4534 \\ 3 & \mathrm{cz} & 14.2875 \\ 4 & \mathrm{pz} & -60.9600 \\ 5 & \mathrm{pz} & 0.0000 \\ 6 & \mathrm{pz} & 86.3600 \\ 7 & \mathrm{pz} & 86.5981 \\ 8 & \mathrm{pz} & 87.0744 \\ 9 & \mathrm{pz} & 87.3125 \\ 10 & \mathrm{pz} & 171.4500\end{array}$

$\$$ FEEO TANK 0.R. - (5/16"-0.040")

\$ FEED TANK O.R.

\$ FEED tANK FLANGE 0.R.

$\$$ CONCRETE FLOOR botton (24" thick) $\$$ CONCRETE FLOOR surface

\$ GLOVEBOX bottom surface

\$ GLOVEBOK Stainless steel (3/321")

\$ GLOVEBOX Lead Shield (3/16")

\$ GLOVEBOX Stainless steel (3/32"1)

$\$$ FEED TANK Bottom Flange botton

$\$$ FEED TANK Bottom $F$ lange tap

$\$$ FEED TANK Solution level (10 L)

$\$$ FEED TANK TOp Flange bottom

$\$$ FEED TANK TOp Flange top

$\$$ GLOVEBOX top inside surface

\$ GLOVEBOX Stainless steel (3/32")

$\$$ GLOVEBOX

\$ GLOVEBOX Stainless Steel (3/32")

$\$$ GLOVEBOX Stainless steel (3/32

$\$$ MODEL TOP (GB roof $+100 \mathrm{~cm}$ )
$\$$ MODEL LEFT SIDE (GB side $+100 \mathrm{~cm}$ )

$\$$ MODEL LEFT SIDE (GB side

$\$$ GLOVEBOX Stainless

\$ GLOVEBOX Lead Shield (3/16")

\$ GLOVEBOX Stainless Steel (3/32")

$\$$ GLOVEBOX right inside surface

\$ GLOVEBOX Stainless Steel (3/32")

GLOVEBOX Lead Shield (3/16")

$\$$ GLOVEBOX Stainless Steel (3/32")

$\$$ GLOVEBOX Stainless Steel (3/32"1)

$\$$ MODEL RACK SIDE (GB back $+100 \mathrm{~cm}$ )

\$ MODEL BACK SIDE (GB back

$\$$ GLOVEBOX back surface

\$ GLOVEBOX Stainless Steel (3/3
$\$$ GLOVEBOX Lead Shield (3/16")

\$ GLOVEBOX Lead Shield (3/16")

$\$$ GLOVEBOX Stainless Steel (3/32

\$ GLOVEBOX Stainless Steel (3/32")

$\$$ GLOVEBOX Lead Shield (3/16")

\$ GLOVEBOX Stainless steel (3/32")

$\$$ MODEL FRONT SIDE (GB front +96")

$\$ 4.5$ ' High $n$ shield

$\$+2.5$ ' Top $n$ shield

$\$$ Left End $n$ Shield

$\$$ Right End $n$ Shield

3/4" n Shield Material outside

$\$ 4$.0" n shield Material outside

$\$ 3 / 4 "$ n Shield Material outside

\$ Operator side $n$ Shield

$\$ 3 / 4^{\prime \prime}$ n Shield Material outside $\$ 1 / 8 "$ n Shield Material outside $\$ 5.0 " \mathrm{n}$ Shield Material outside $\$ 1 / 8 "$ n Shield Material outside

\$1/8" n shield Material inside

$\$$ Operator side $n$ shield

$\$$ Operator Side n Shield

$\$ 1.0^{\prime \prime}$ n Shield Material outside

$\$ 3 / 4^{\prime \prime}$ n Shield Material outside

\$ Operator side $n$ shield

$\$ 3 / 4^{1 *} \mathrm{n}$ Shield Material outside

$\$ 1 / 8^{14} \mathrm{n}$ Shield Material outside

$\$ 1.014$ Shield Material outside

$\$ 3 / 4^{1 *} n$ Shield Material outside

$\$ 1 / 8^{\prime \prime} n$ Shield Haterial inside

$\$$ Operator side $n$ Shield 


$\begin{array}{llr}57 & \text { pz } & 949.96 \\ 58 & p z & 960.12 \\ 59 & p x & -561.34 \\ 60 & p x & -541.02 \\ 61 & \text { px } & 251.46 \\ 62 & \text { py } & -735.3078 \\ 63 & \text { py } & -735.0125 \\ 64 & \text { py } & 327.6338\end{array}$

$\$$ Ceil ing surface [H-2-16174] $\$ 4$ inch concrete roof [H-2-16174]

$\$ 8$ inch concrete wall left side

$\$$ Left Wall Surface $[\mathrm{H}-2-16140]$

$\$ 8$ inch concrete wall right side

$\$ 18$ Gauge Metal $(.051611 *(4.0 / 1.8)=>2913 \mathrm{~cm})$

Back Wall Surf

$\$ 18$ Gauge Metal $\left(.0516^{11 \star}(4.0 / 1.8)=>.2913 \mathrm{~cm}\right)$

mode $\quad \mathrm{p}$ imp:p $149 \mathrm{r} 0$

c wgt $=3500 * 3.169 \mathrm{E}+8=1.109 \mathrm{E}+12$ Low $240 \mathrm{Pu}$

c wgt $=3500 * 1.115 \mathrm{E}+9=3.903 \mathrm{E}+12$ Mid $240 \mathrm{Pu}$

c wgt $=3500 * 1.682 \mathrm{E}+9=5.887 \mathrm{E}+12$ High $240 \mathrm{Pu}$

sdef cel $=1$ erg $=d 5 \quad$ pos $=000$ rad $=d 3$

ext $=d 4 \quad$ axs $=001$ wgt $=5.887 \mathrm{E}+12$

sc2 (alpha, $n$ ) and fission spectrum

$\mathrm{si2} s \quad 35 \quad 36$

$\mathrm{sp2} \quad 0.504 \quad 0.496$

radius

$\begin{array}{ll}\text { si3 } & 7.7610 \\ \text { sp3 } & -211\end{array}$

sp3 -211

$\begin{array}{lll}\text { sc4 } & \text { extent } & \\ \text { si4 } & 171.7200 & 224.5663\end{array}$

$-210$

sc5 photon source from ORLGEN2

TOTAL PHOTON SPECTRUM

\begin{tabular}{lll}
\multicolumn{1}{c}{ si5 } & sp5 & \multicolumn{2}{c}{ sb5 } & \\
0.0 & 0.0 & 0 \\
$1.5000 \mathrm{e}-02$ & $8.9240 \mathrm{e}+08$ & 200790 \\
$2.5000 \mathrm{e}-02$ & $4.9830 \mathrm{e}+07$ & 31144 \\
$3.7500 \mathrm{e}-02$ & $4.6240 \mathrm{e}+06$ & 6503 \\
$5.7500 \mathrm{e}-02$ & $7.3350 \mathrm{e}+08$ & 2425134 \\
$8.5000 \mathrm{e}-02$ & $6.1780 \mathrm{e}+05$ & 4464 \\
$1.2500 \mathrm{e}-01$ & $7.0030 \mathrm{e}+05$ & 10942 \\
$2.2500 \mathrm{e}-01$ & $7.9320 \mathrm{e}+04$ & 4016 \\
$3.7500 \mathrm{e}-01$ & $1.2800 \mathrm{e}+05$ & 18000 \\
$5.7500 \mathrm{e}-01$ & $1.7600 \mathrm{e}+04$ & 5819 \\
$8.5000 \mathrm{e}-01$ & $5.1220 \mathrm{e}+03$ & 3701 \\
$1.2500 \mathrm{e}+00$ & $1.6370 \mathrm{e}+02$ & 256 \\
$1.7500 \mathrm{e}+00$ & $7.2840 \mathrm{e}+01$ & 223 \\
$2.2500 \mathrm{e}+00$ & $4.1830 \mathrm{e}+01$ & 212 \\
$2.7500 \mathrm{e}+00$ & $2.4080 \mathrm{e}+01$ & 182 \\
$3.5000 \mathrm{e}+00$ & $2.1360 \mathrm{e}+01$ & 262 \\
$5.0000 \mathrm{e}+00$ & $9.0380 \mathrm{e}+00$ & 226 \\
$7.0000 \mathrm{e}+00$ & $1.0250 \mathrm{e}+00$ & 50 \\
$1.1000 \mathrm{e}+01$ & $1.1690 \mathrm{e}-01$ & 14 \\
TOTAL & $1.6820 \mathrm{e}+09$ &
\end{tabular}

c TOTAL

$$
1.6820 \mathrm{e}+09
$$

00790

6503

2425134

4464

4016

8000

819

56

212

182

14

sc35 (alpha, $n$ ) neutron source on OXYGEN from Jacom and Liskien

c Energy of the alpha particles $=5.5 \mathrm{MeV}$

$\begin{array}{cc}\text { si35 } & \text { sp35 } \\ 0.0 & 0.00 \\ 0.1 & 0.68 \\ 0.2 & 0.76\end{array}$

0.00

$0.2 \quad 0.76$

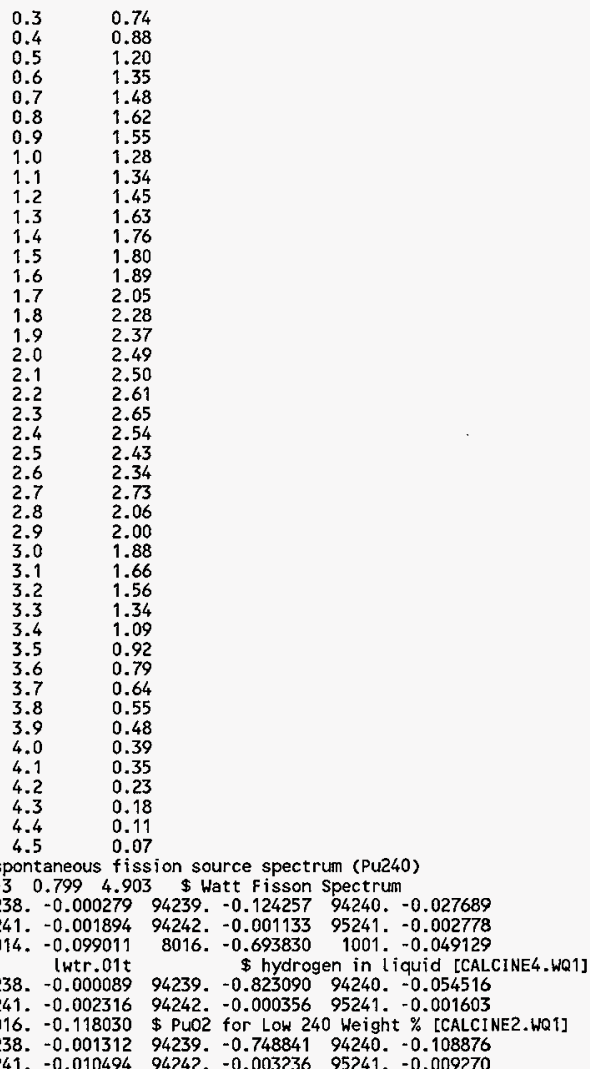

$\mathrm{sp} 36$

m1

$c$
$m 2$ 
8016. -0.117971 \$ PLO2 for Med 240 Weight \% [CALCINE2. WQ1] 94238. $-0.00155994239,-0.69358194240 .-0.154553$ 94241. -0.010569 94242. -0.00632495241 . -0.015507 8016. -0.117907 \$ PUO2 for High 240 Weight \% [CALCINE2.WQ1] 14000. $-0.376753 \quad 5010 .-0.0079865011 .-0.032386$ 8016. $-0.540198 \$ 7740$ PYREX TYPe I Glass [CALCINE1. WQ1] m6 26000. -0.74 24000. -0.18 28000. -0.08 \$ SS 304L Flanges m7 26000. -0.98 28000. -0.02 \& Stainless steel Glovebox $m 882000,-1.00 \quad \$$ Lead Glovebox Shield $m 926000$. $-1 \quad \$$ SS $310 \mathrm{~s}$

m10 7014:-.78084 8016. $-0.2094618000 .35 \mathrm{c}-0.00934$ 1001. -.00036 \& Air

c mt10 Iwtr.01t \$ hydrogen in liquid, gas

m11 13027. -.034 20000. -0.044 26000. -0.014 1001. -.001 8016. -0.532 14000. -0.337 11023. - $.029 \$$ Concrete (common Port land) ARH-600 II.F.1-3 c $m t 11$ poly.01t \$ hydrogen in solid m12 6000. -0.5925 5010. -0.0590 5011. $-0.2410 \$$ Neutron 1001. -0.0854 14000. -0.0101 8016. -0.0076 \$ Shield 26000 - $0.003813027-0.0004$ 25055 -0.0002 \$210

$c$ mt12 poly.01t grph.01t \$ hydrogen, carbon in solid $(1.19$ $\mathrm{g} / \mathrm{cm}^{3}$

8016. $-0.36496000 .-0.3439$ 13027. -0.1906 \$ Neutron 1001. $-0.078420000 .-0.0123$ 14000. $-0.0009 \$$ Shield 5010. -0.00138 5011. -0.0056212000 . $-0.0006 \$ 207$ 11023 . -0.0006 39089. -0.0005 26000. -0.0001

19000, -0.0001 16032. $-0.0001 \$ Y 89$ substituted for $\mathrm{sr}$. $c$ mt13 poly.01t grph.01t $\$$ hydrogen, carbon in solid (1.4

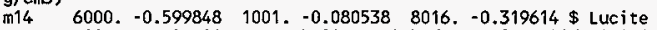
c mt14 poly.01t grph.01t \$ hydrogen in solid $(1.185 \mathrm{~g} / \mathrm{cm})$ Lucite

$\$$ hydrogen in sol id $(1.185 \mathrm{~g} / \mathrm{cm} 3)$

$\begin{array}{lll}14000 & -0.392603 & \$ 7740 \text { PYREX Type I Glass [no Boron } \\ 11023 .-0.030923 & 13027 .-0.012685 & 19000 .-0.000865\end{array}$

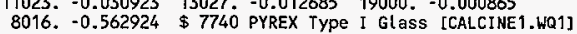

m16 6000. -0.755751 1001. $-0.0554918016 .-0.188758 \$$ Lexan Lexan $m$ t16 poly.01t grph.01t $\$$ hydrogen in solid $(1.2 \mathrm{~g} / \mathrm{cm} 3)$

c Photon dose conversion factors from ANS 6.1.1 1991

$\begin{array}{lllllll}\text { de } 0 \quad \log & .01 & .015 & .02 & .03 & .04 & .05\end{array}$

$\begin{array}{cccccc}.06 & .08 & .10 & .15 & .20 & .30 \\ .40 & .50 & .60 & .80 & 1.0 & 1.5 \\ 2.0 & 3.0 & 4.0 & 5.0 & 6.0 & 8.0\end{array}$

dfo

$10.0 \quad 12.0$

$\log 2.232 e-5 \quad 5.652 e-5 \quad 8.568 e-5 \quad 1.184 e-4 \quad 1.314 e-4 \quad 1.382 e-4$

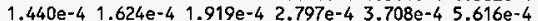

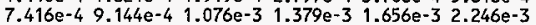
$2.758 e-3 \quad 3.672 e-3 \quad 4.500 e-3 \quad 5.292 e-3 \quad 6.012 e-3 \quad 7.488 e-3$ $8.892 \mathrm{e}-3 \quad 1.040 \mathrm{e}-2$

fc4 Photon Dose behind Neutron Shield, Right side Leak Around $f 4: \mathrm{p} 39$ \$ Operator behind Neutron Shield

cf $4 \quad 20$

skyshine

fc14 Photon Dose behind Neutron Shield, Front Lower $n$ Shield Leak Through f14:p 39 \$ Operator behind Neutron Shield

cf14 $25 \quad$ \$ Flagging Cells for leak-through,

skyshine

fc24 Photon Dose behind Neutron Shield, Front Upper $n$ Shield Leak Through f24:p 39 \$ Operator behind Neutron Shield

282 \$ Flagging Cells for leak-through,

fc34 Photon Dose behind Neutron Shield, Left Lower $n$ Shield Leak Through

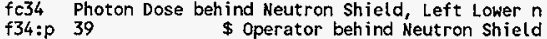

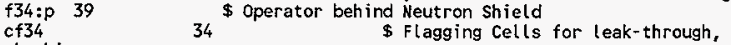

skyshine

fe44 Photon Dose behind Neutron Shield, Left Upper $n$ Shield Leak Through f44:p 39 \$ Operator behind Neutron Shield

cf44 37 Flagging Cells for leak-through,

skyshine

fc54 Photon Dose behind Neutron Shield, Roof Reflection

f54:p $39 \quad \$$ Operator behind Neutron Shield

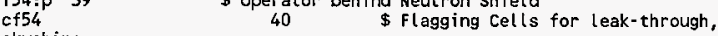

skyshine

fc64 Photon Dose behind Neutron Shield, steel Wall Back Reflection

f64:p 39 \$ Operator behind Neutron Shield

cf64 45 \$ Flagging Cells for leak-through,

skyshine

fC74 Photon Dose behind Neutron Shield, Concrete Floor Effect

$f 74: \mathrm{p} 39 \quad$ \$ Operator behind Neutron Shield

cf74 50 \$ Flagging Cells

fc84 Photon Dose behind Neutron Shield, Sum of All Cells FLAGgING!!

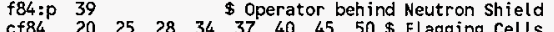

$\mathrm{fc} 5$ Photon dose at $30 \mathrm{~cm}$ from outside surface of 6 in pipe

fc5

$\mathrm{f} 5: \mathrm{p} \quad 0 \quad 38.4534200 .0 \quad 0 \quad 38.4534 \mathrm{~cm}$

fc15 Photon dose at $30 \mathrm{~cm}$ from outside surface of Glovebox

c Total Dist from outside $=30.0+83.5025=113.5025 \mathrm{~cm}$

f15: $\mathrm{p} \quad 0113.5025200 .0$ 0

fc25 Photon dose at 5 feet from outside surface of Glovebox

c Total Dist from outside $=152.8+83.5025=235.9025 \mathrm{~cm}$

f25: 0235.9025200 .00

fc35 Photon dose at 6 in. in front of lower $n$ shield

c Total Dist from outside $=191.4525-6 * 2.54=176.2125 \mathrm{~cm}$

f35: $p \quad 0$ 176.2125 $91.44 \quad 0$

fc45 Photon dose in middle of outer 1/8" SST n Shield

c Total Dist from outside $=(191.7700+192.0875) / 2=191.9288 \mathrm{~cm}$

f45:P $0191.9288 \quad 91.44 \quad 0$

fc55 Photon dose in middle of $5.0^{\prime \prime}$ lower $n$ Shield

c Total Dist from outside $=(192.0875+204.7875) / 2=198.4375 \mathrm{~cm}$

f55:p $\quad 0198.4375 \quad 91.44 \quad 0$

fc65 Photon dose in middle of inner 1/8" stainless steel n shield

$c$ Total Dist from outside $=(204.7875+205.1050) / 2=204.9462 \mathrm{~cm}$

f65:p $0204.9462 \quad 91.44 \quad 0$

fc75 Photon dose at $6 \mathrm{in}$. behind inside lower $\mathbf{n}$ Shield

c Total Dist from outside $=205.4225+6 * 2.54=220.6225 \mathrm{~cm}$

$f 75: p \quad 0220.6625 \quad 91.44 \quad 0$ 


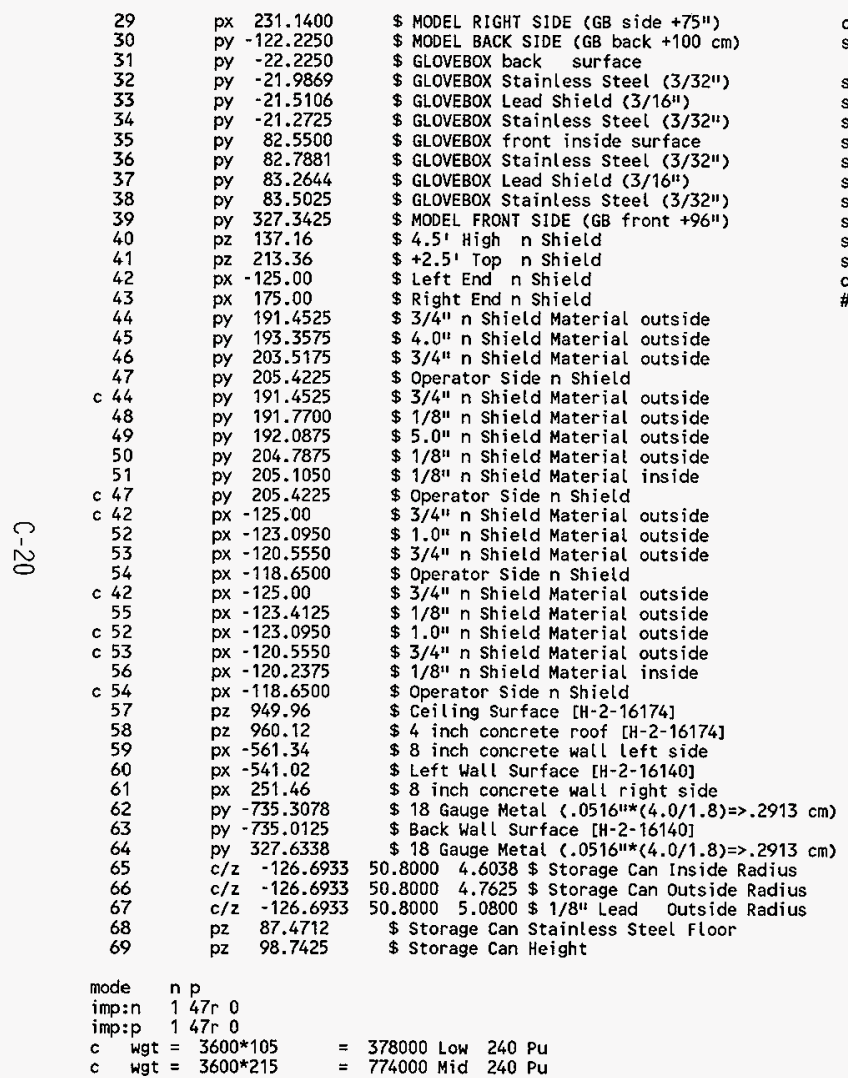

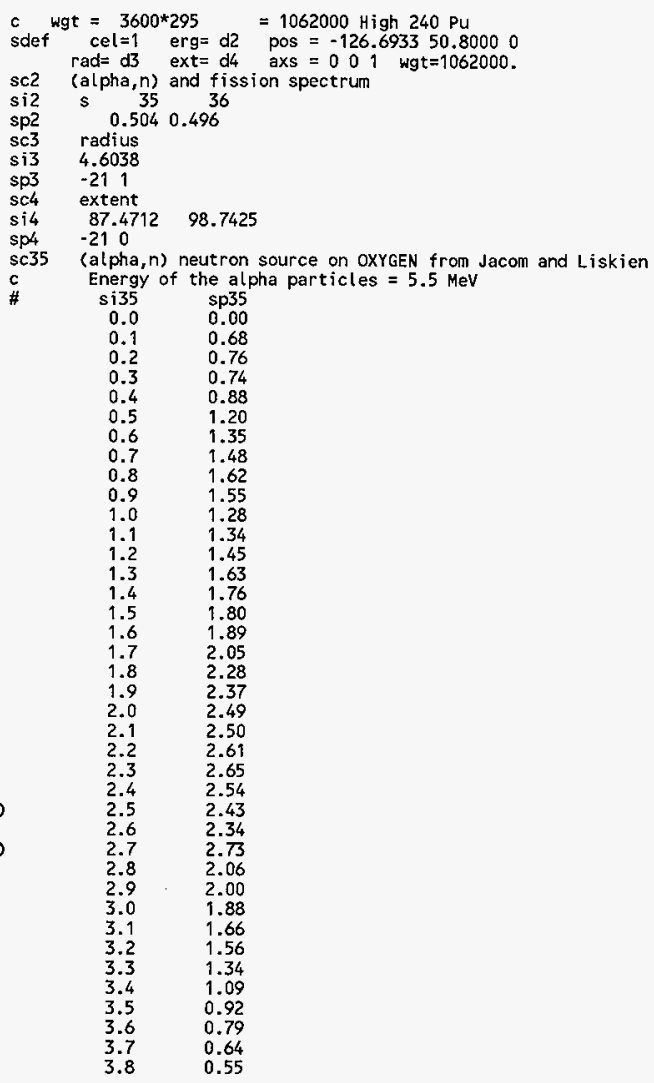




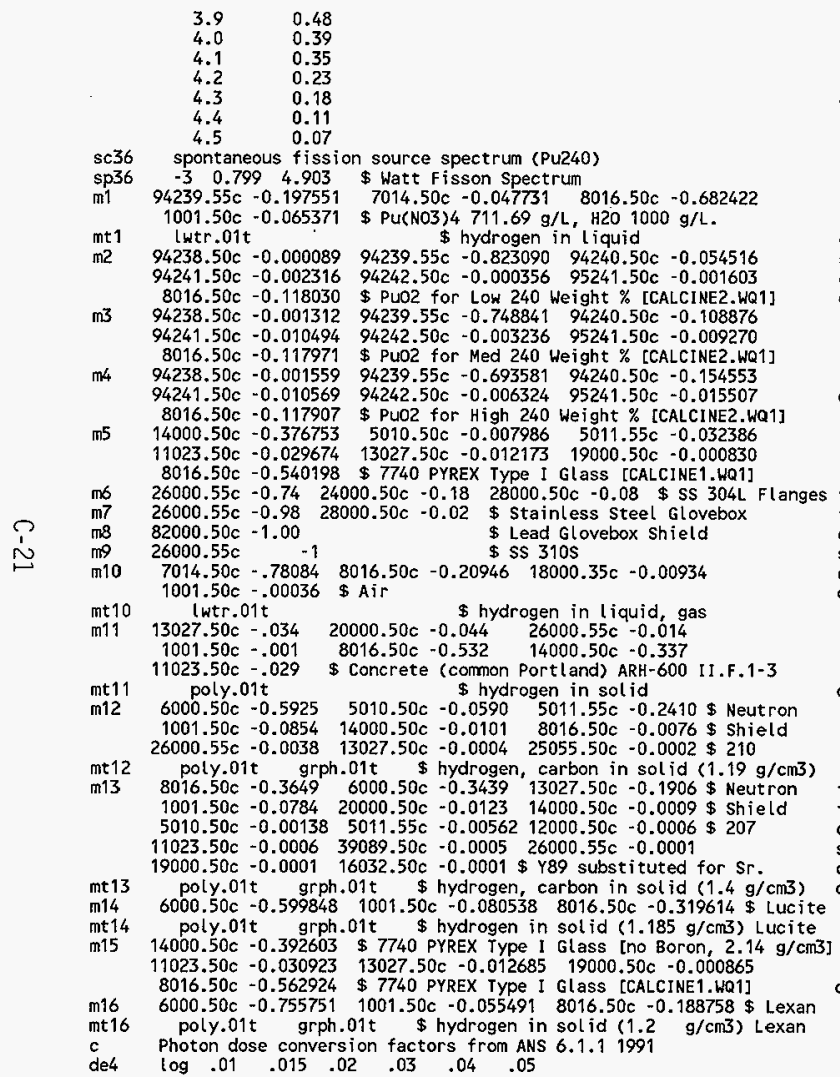

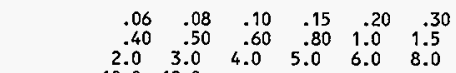

df4

$10.0 \quad 12.0$

$\log 2.232 e-5 \quad 5.652 e-5 \quad 8.568 e-5 \quad 1.184 e-4 \quad 1.314 e-4 \quad 1.382 e-4$

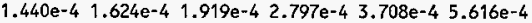

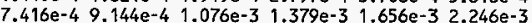

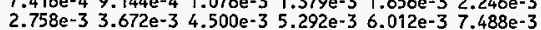
$2.758 \mathrm{e}-3$
$8.892 \mathrm{e}-3$
$1.040 \mathrm{e}-2$

fc4 Photon Dose behind Neutron Shield, Right Side Leak Around

$\mathrm{f4: \textrm {P }} \quad 37 \quad$ \$ Operator behind Neutron Shield

$\begin{array}{lll}f 4: p & 37 & \$ \text { Operator behind Neutron Shield } \\ c f 4 & 18 & \text { \$ Flagging Cells for leak-through, } \\ \text { skyshine } & \end{array}$

c Photon dose conversion factors from ANs 6.1.1 1991

de14 $\log \begin{array}{llllll}.01 & .015 & .02 & .03 & .04 & .05\end{array}$

$\begin{array}{cccccc}.06 & .08 & .02 & .03 & .04 & .05 \\ .40 & .50 & .60 & .80 & . .20 & .30 \\ 2.0 & 3.0 & 4.0 & 5.0 & 6.0 & 1.5 \\ & & & \end{array}$

df14 $10.0 \quad 12.0$

$\log 2.232 e-5 \quad 5.652 e-5 \quad 8.568 e-5 \quad 1.184 e-4 \quad 1.314 e-4 \quad 1.382 e-4$

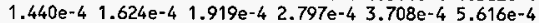

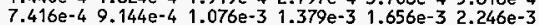

$2.758 \mathrm{e}-3 \quad 3.672 \mathrm{e}-3 \quad 4.500 \mathrm{e}-3 \quad 5.292 \mathrm{e}-3 \quad 6.012 \mathrm{e}-3 \quad 7.488 \mathrm{e}-3$ $8.892 \mathrm{e}-31.040 \mathrm{e}-2$

fc14 Photon Dose behind Neutron Shield, Front Lower $n$ Shield Leak Through f14:p 37 Operator behind Neutron shield

$23 \quad \$$ Flagging Cells for leak-through,

skyshine

c Photon dose conversion factors from ANS 6.1 .11991

de24 log $\begin{array}{llllll}.01 & .015 & .02 & .03 & .04 & .05\end{array}$

$\begin{array}{llllll}.06 & .08 & .02 & .03 & .04 & .05 \\ .40 & .50 & .60 & .15 & .20 & .30\end{array}$

$\begin{array}{llllll}.40 & .50 & .60 & .80 & 1.0 & 1.5 \\ 2.0 & 3.0 & 4.0 & 5.0 & 6.0 & 8.0\end{array}$

df24 $\quad \log ^{10.0} 2.232 e^{-5} \quad 5.652 e-5 \quad 8.568 e-5 \quad 1.184 e-4 \quad 1.314 e-4 \quad 1.382 e-4$

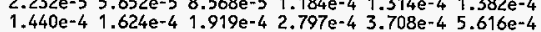

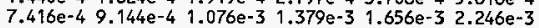

$2.758 \mathrm{e}-3 \quad 3.672 \mathrm{e}-3 \quad 4.500 \mathrm{e}-3 \quad 5.292 \mathrm{e}-3 \quad 6.012 \mathrm{e}-3 \quad 7.488 \mathrm{e}-3$ $8.892 \mathrm{e}-31.040 \mathrm{e}-2$

fc24 Photon Dose behind Neutron Shield, Front Upper $n$ Shield Leak Through f24:p 37 \$ Operator behind Neutron Shield

cf24 26 \$ Flagging Cells for leak-through, skyshine

c Photon dose conversion factors from ANS 6.1.1 1991

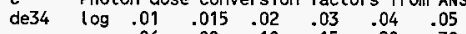

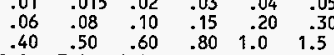

$\begin{array}{llllll}.40 & .50 & .60 & .80 & 1.0 & 1.5 \\ 2.0 & 3.0 & 4.0 & 5.0 & 6.0 & 8.0\end{array}$

$\begin{array}{rr}2.0 & 3.0 \\ 10.0 & 12.0\end{array}$

df34 $\log 2.232 \mathrm{e}-5 \quad 5.652 \mathrm{e}-5 \quad 8.568 \mathrm{e}-5 \quad 1.184 \mathrm{e}-4 \quad 1.314 \mathrm{e}-4 \quad 1.382 \mathrm{e}-4$

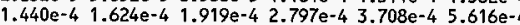

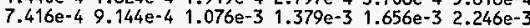
$2.758 \mathrm{e}-3 \quad 3.672 \mathrm{e}-3 \quad 4.500 \mathrm{e}-3 \quad 5.292 \mathrm{e}-3 \quad 6.012 \mathrm{e}-3 \quad 7.488 \mathrm{e}-3$ $8.892 \mathrm{e}-3$ 1.040e-2 
fc34 Photon Dose behind Neutron Shield, Left Lower $n$ Shield Leak Through $\begin{array}{lll}\mathrm{f34:p} & 37 & \text { \$ Operator behind Neutron Shield } \\ \mathrm{cf3} 34 & 32 & \text { \$ Flagging Cells }\end{array}$ skyshine

c Photon dose conversion factors from ANS 6.1.1 1991

de44 $\log \begin{array}{llllll}.01 & .015 & .02 & .03 & .04 & .05\end{array}$

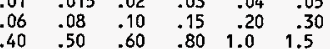

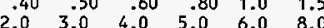

df44

$\log 2.232 e-5 \quad 5.652 e-5 \quad 8.568 e-5 \quad 1.184 e-4 \quad 1.314 e-4 \quad 1.382 e-4$

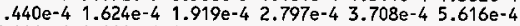

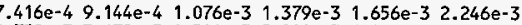
$2.758 \mathrm{e}-3 \quad 3.672 \mathrm{e}-3 \quad 4.500 \mathrm{e}-3 \quad 5.292 \mathrm{e}-3 \quad 6.012 \mathrm{e}-3 \quad 7.488 \mathrm{e}-3$ $8.892 \mathrm{e}-31.040 \mathrm{e}-2$

fc44 Photon Dose behind Neutron Shield, Left Upper $n$ Shield Leak Through f44:p 37 \$ Operator behind Neutron shield

cf44 35 \$ Flagging Cells for leak-through, skyshine

c Photon dose conversion factors from ANS 6.1 .11991

$\begin{array}{cccccc}.01 & .015 & .02 & .03 & .04 & .05 \\ .06 & .08 & .10 & .15 & .20 & .30 \\ .40 & .50 & .60 & .80 & 1.0 & 1.5 \\ 2.0 & 3.0 & 4.0 & 5.0 & 6.0 & 8.0\end{array}$

df54 $\log 2.232 e-5 \quad 5.652 e-5 \quad 8.568 e-5 \quad 1.184 e-4 \quad 1.314 e-4 \quad 1.382 e-4$ $1.440 \mathrm{e}-4 \quad 1.624 \mathrm{e}-4 \quad 1.919 \mathrm{e}-4 \quad 2.797 \mathrm{e}-4 \quad 3.708 \mathrm{e}-4 \quad 5.616 \mathrm{e}-4$

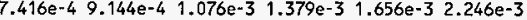
$2.758 \mathrm{e}-3 \quad 3.672 \mathrm{e}-3 \quad 4.500 \mathrm{e}-3 \quad 5.292 \mathrm{e}-3 \quad 6.012 \mathrm{e}-3 \quad 7.488 \mathrm{e}-3$ $8.892 \mathrm{e}-3 \quad 1.040 \mathrm{e}-2$

fc54 Photon Dose behind Neutron shield, Roof Reflection
f54:p 37

$\begin{array}{ccc}\text { f54:p } & 37 & \text { \$Operator behind Neutron Shield } \\ \text { cf54 } & 38 & \text { \$ Flagging Cells for leak-through }\end{array}$

skyshine

c Photon dose conversion factors from ANS 6.1 .11991

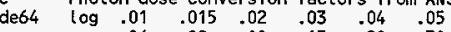

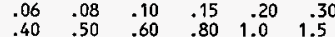

$\begin{array}{cccccc}2.0 & 3.0 & 4.0 & 5.0 & 6.0 & 8.0\end{array}$

$10.0 \quad 12.0$

df64 $\log 2.232 \mathrm{e}-5 \quad 5.652 \mathrm{e}-5 \quad 8.568 \mathrm{e}-5 \quad 1.184 \mathrm{e}-4 \quad 1.314 \mathrm{e}-4 \quad 1.382 \mathrm{e}-4$ $1.440 \mathrm{e}-4 \quad 1.624 \mathrm{e}-4 \quad 1.919 \mathrm{e}-42.797 \mathrm{e}-4 \quad 3.708 \mathrm{e}-4 \quad 5.616 \mathrm{e}-4$ $7.416 \mathrm{e}-4 \quad 9.144 \mathrm{e}-4 \quad 1.076 \mathrm{e}-3 \quad 1.379 \mathrm{e}-3 \quad 1.656 \mathrm{e}-32.246 \mathrm{e}-3$ $2.758 \mathrm{e}-3 \quad 3.672 \mathrm{e}-3 \quad 4.500 \mathrm{e}-3 \quad 5.292 \mathrm{e}-3 \quad 6.012 \mathrm{e}-3 \quad 7.488 \mathrm{e}-3$ $8.892 \mathrm{e}-3$ 1.040e-2

fc64 Photon Dose behind Neutron Shield, Steel Wall Back Reflection

$\begin{array}{lll}\text { f664:p } 37 & \text { \$ Operator behind Neutron Shield } \\ \text { cf } 64 & 43 \quad \$ \text { Flagging Cells for leak-through, }\end{array}$ skyshine

Photon dose conversion factors from ANS 6.1.1 1991

de74 $\log \begin{array}{llllll}.01 & .015 & .02 & .03 & .04 & .05\end{array}$

$\begin{array}{llllll}.06 & .08 & .10 & .15 & .20 & .30 \\ .40 & .50 & .60 & .80 & 1.0 & 1.5 \\ 2.0 & 3.0 & 4.0 & 5.0 & 6.0 & 8.0\end{array}$

$10.0 \quad 12.0$

df74 $\log \quad 2.232 e-5 \quad 5.652 e-5 \quad 8.568 e-5 \quad 1.184 e-4 \quad 1.314 e-4 \quad 1.382 e-4$ $1.440 \mathrm{e}-4 \quad 1.624 \mathrm{e}-4 \quad 1.919 \mathrm{e}-42.797 e-4 \quad 3.708 \mathrm{e}-4 \quad 5.616 e-4$

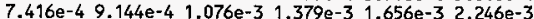
$2.758 \mathrm{e}-3 \quad 3.672 \mathrm{e}-3 \quad 4.500 \mathrm{e}-3 \quad 5.292 \mathrm{e}-3 \quad 6.012 \mathrm{e}-3 \quad 7.488 \mathrm{e}-3$ $8.892 \mathrm{e}-31.040 \mathrm{e}-2$

fc74 Photon Dose behind Neutron Shield, Concrete Floor Effect f74:p 37 \$ Operator behind Neutron Shield

$48 \$$ Flagging Cells for leak-through, skyshine

c Photon dose conversion factors from ANS 6.1.1 1991

de84 $\log \begin{array}{llllll}.01 & .015 & .02 & .03 & .04 & .05\end{array}$

$\begin{array}{llllll}.06 & .08 & .10 & .15 & .20 & .30 \\ .40 & .50 & .60 & .80 & 1.0 & 1.5\end{array}$

$\begin{array}{llllll}2.0 & 3.0 & 4.0 & 5.0 & 6.0 & 8.0\end{array}$

$10.0 \quad 12.0$

df84 $\log 2.232 e-5 \quad 5.652 e-5 \quad 8.568 e-5 \quad 1.184 e-4 \quad 1.314 e-4 \quad 1.382 e-4$

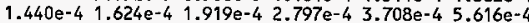

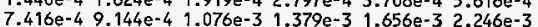

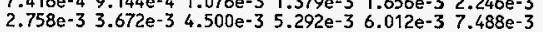
$8.892 \mathrm{e}-31.040 \mathrm{e}-2$

fc84 Photon Dose behind Neutron Shield, sum of All Cells FLAGGING! f84:p $37 \quad$ \$Operator behind Neutron Shield

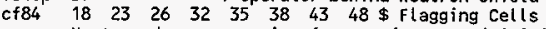

c Neutron dose conversion factors from ANS 6.1 .11991

de $0 \quad 2.50 \mathrm{e}-08 \quad 1.00 \mathrm{e}-07 \quad 1.00 \mathrm{e}-06 \quad 1.00 \mathrm{e}-05 \quad 1.00 \mathrm{e}-04 \quad 1.00 \mathrm{e}-03$ $\begin{array}{llllll}1.00 \mathrm{e}-02 & 2.00 \mathrm{e}-02 & 5.00 \mathrm{e}-02 & 1.00 \mathrm{e}-01 & 2.00 \mathrm{e}-01 & 5.00 \mathrm{e}-01\end{array}$

$\begin{array}{llllll}1.00 \mathrm{e}+00 & 1.50 \mathrm{e}+00 & 2.00 \mathrm{e}+00 & 3.00 \mathrm{e}+00 & 4.00 \mathrm{e}+00 & 5.00 \mathrm{e}+00\end{array}$

$\begin{array}{lllll}6.00 \mathrm{e}+00 & 7.00 \mathrm{e}+00 & 8.00 \mathrm{e}+00 & 1.00 \mathrm{e}+01 & 1.40 \mathrm{e}+01\end{array}$

df0 $\quad 1.440 \mathrm{e}-03 \quad 1.584 \mathrm{e}-03 \quad 1.735 \mathrm{e}-03 \quad 1.606 \mathrm{e}-03 \quad 1.490 \mathrm{e}-03 \quad 1.379 \mathrm{e}-03$ $1.631 \mathrm{e}-03 \quad 2.113 \mathrm{e}-03 \quad 3.924 \mathrm{e}-03 \quad 7.128 \mathrm{e}-03 \quad 1.390 \mathrm{e}-02 \quad 3.132 \mathrm{e}-02$

$5.148 \mathrm{e}-02 \quad 6.588 \mathrm{e}-02 \quad 7.704 \mathrm{e}-02 \quad 9.504 \mathrm{e}-02 \quad 1.080 \mathrm{e}-01 \quad 1.177 \mathrm{e}-01$

$1.249 \mathrm{e}-01 \quad 1.314 \mathrm{e}-01 \quad 1.368 \mathrm{e}-01 \quad 1.476 \mathrm{e}-011.728 \mathrm{e}-01$

fc204 Neutron Dose behind Neutron shield, Right side Leak Around f204:n $37 \quad$ \$ Operator behind Neutron Shield

fm204 2.0 \$ Factor of 2 times the ANS 6.1 .11991 standard. ef204 18 $\$$ Flagging Cells for leak-through, skyshine

fc214 Neutron Dose behind Neutron Shield, Front Lower $n$ Shield Leak Through

$f 214: \mathrm{n} 37$ \$ Operator behind Neutron Shield

fm214 $2.0 \quad$ \$ Factor of 2 times the ANS 6.1.1 1991 standard.

cf214 $23 \quad \$$ Flagging Cells for leak-through,

skyshine

fc224 Neutron Dose behind Neutron Shield, Front Upper $n$ Shield Leak Through

f224:n 37 \$ Operator behind Neutron Shield

fm224 $2.0 \quad$ \$ Factor of 2 times the ANS 6.1 .11991 standard.

cf224

26

$\$$ Flagging Cells for leak-through

fc234 Neutron Dose behind Neutron Shield, Left Lower $n$ Shield Leak

Through

f234:n 37 \$ Operator behind Neutron shield

fm234 2.0 \$ Factor of 2 times the ANS 6.1 .11991 standard. 
cf234

skyshine

32

$\$$ Flagging Cells for leak-through,

fc244 Neutron Dose behind Neutron Shield, Left Upper $n$ Shield Leak

Through

$\$$ operator behind Neutron Shield

cf244

cf244

$\$$ Factor of 2 times the ANS 6.1 .11991 Standard.

fc254 Neutron Dose behind Neutron Shield, Roof Reflection

f254:n 37 \$ Operator behind Neutron Shield

$\begin{array}{ccc}\text { fm254 } & 2.0 & \$ \text { Operator behind Neutron Shield } \\ \text { cf254 } & \text { \$ Factor of } 2 \text { times the ANS } 6.1 .1 \text { 1991 standard. }\end{array}$

fc264 Neutron Dose behind Neutron Shield, Steel Wall Back Reflection

f264:n 37 \$ Operator behind Neutron Shield

fm264 2.0 \$ Factor of 2 times the ANS 6.1 .11991 standard.

cf264 43 \$ Flagging Cells for leak-through,

skyshine

$\begin{array}{ll}\text { fc274 Neutron Dose behind Neutron Shield, Concrete Floor } \\ \text { f274:n } 37 & \text { \$ Operator behind Neutron Shield }\end{array}$

$\begin{array}{lll}f 274: \mathrm{n} & 37 & \text { \$ Operator behind Neutron Shield } \\ \mathrm{fm} 274 & 2.0 & \text { \$ Factor of } 2 \text { times the ANS } 6.1 .11991 \text { standard. }\end{array}$

$\mathrm{fm} 274$
$\mathrm{cf} 274$$\quad \begin{aligned} & \text { \$ Factor of } 2 \text { times the ANS } 6.1 .1 \text { 1991 standard. } \\ & 48 \$ \mathrm{~F} \text { lagging Cells for leak-through }\end{aligned}$

skyshine

fc284 Neutron Dose behind Neutron shield, Sum of All Cells FLAGGING!!

f284:n 37 Operator behind Neutron Shield

fm284 $2.0 \quad$ Factor of 2 times the ANS 6.1 .19991 standard. $\begin{array}{llllllllll}\text { cf284 } & 18 & 23 & 26 & 32 & 35 & 38 & 43 & 48 & \$\end{array}$ skyshine

fc5 dose at $30 \mathrm{~cm}$ from outside surface of 6 in. pipe

c Total Dist from outside $=30.0+50.80+5.08=85.88 \mathrm{~cm}$

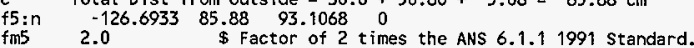

f 15 2.0 $30 \mathrm{~cm}$ Factor of 2 times the ANS 6.1

$\mathrm{fc} 15$ dose at $30 \mathrm{~cm}$ from outside surface of Glovebox
$c$

$\begin{array}{lll}c & \text { Total Dist from outside }=30.0+ \\ \text { f15:n } & -126.6933 \quad 113.502593 .1068 \quad 0\end{array}$

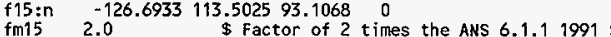

fm15 2.0 \$ Factor of 2 times the ANS 6.1.1 1991 standard.

fe25 dose at 5 feet from outside surface of Glovebox

c Total Dist from outside $=152.8+83.5025=235.9025 \mathrm{~cm}$

f25:n $\quad-126.6933235 .902593 .1068$ o

$f m 252.0$ \& Factor of 2 times the ANS 6.1 .11991 standard.

fc35 Neutron dose at $6 \mathrm{in}$. in front of lower n shield

c Total Dist from outside $=191.4525-6 * 2.54=176.2125 \mathrm{~cm}$

f35:n $\quad 0176.2125 \quad 91.44 \quad 0$

fm35 2.0 Factor of 2 times the ANS 6.1 .11991 standard.

fc45 Neutron dose in middle of outer $1 / 8$ " SST $n$ shield

c Total Dist from outside $=(191.7700+192.0875) / 2=191.9288 \mathrm{~cm}$

f45:n $0191.9288 \quad 91.44 \quad 0$

fm45 $2.0 \quad \$$ Factor of 2 times the ANS 6.1 .11991 standard. $\mathrm{fc55}$ Neutron dose in middle of $5.0^{\prime \prime}$ lower $\mathbf{n}$ Shield

c Total Dist from outside $=(192.0875+204.7875) / 2=198.4375 \mathrm{~cm}$ f55:n 0 198.4375 $91.44 \quad 0$

fm55 2.0 \$ Factor of 2 times the ANS 6.1.1 1991 Standard.

fc65 Neutron dose in middle of inner 1/8" Stainless Steel $n$ Shield f65:n $\quad 0204.9462 \quad 91.44 \quad 0$

fn65 2.0 Factor of 2 times the ANS 6.1 .11991 Standard.

fc75 Neutron dose at 6 in. behind inside lower $\mathbf{n}$ Shield

c Total Dist from outside $=205.4225+6 * 2.54=220.6225 \mathrm{~cm}$

f75:n $\quad 0220.6625 \quad 91.44 \quad 0$

$f m 75 \quad 2.0 \quad \$$ Factor of 2 times the ANS 6.1 .11991 standard $f c 85$ Neutron dose at $6 \mathrm{in}$. in front of upper $n$ Shield

c Total Dist from outside $=191.4525-6 * 2.54=176.2125 \mathrm{~cm}$

f85:n $\quad 0176.2125 \quad 182.88 \quad 0$

fm85 2.0 \$ Factor of 2 times the ANS 6.1 .11991 standard. fc95 Neutron dose in middle of outer 3/4" glass $n$ Shield

c Total $D$ ist from outside $=(191.4525+193.3575) / 2=192.4050 \mathrm{~cm}$ f95:n $0192.4050182 .88 \quad 0$

fn95 2.0 Factor of 2 times the ANS 6.1.1 1991 standard.

fc105 Neutron dose in middle of 4.0" upper $n$ Shield

c Total Dist from outside $=(193.3575+203.5175) / 2=198.4375 \mathrm{~cm}$

f105:n 0 198.4375 $182.88 \quad 0$

$f \mathrm{~m} 1052.0 \quad$ Factor of 2 times the ANS 6.1 .11991 standard. f 115 Neutron dose in middle of inner $3 / 4^{\prime \prime}$ glass $n$ shield

Total Dist from outside $=(203.5175+205.4225) / 2=204.4700 \mathrm{~cm}$ f115:n $\quad 0204.4700182 .88$ o

fm115 2.0 \$ Factor of 2 times the ANS 6.1 .11991 standard. fo125 Neutron dose at 6 in. behind inside upper $n$ Shield

c Total Dist from outside $=205.4225+6 * 2.54=220.6225 \mathrm{~cm}$

f125:n $0220.6625182 .88 \quad 0$

fm125 2.0 \$ Factor of 2 times the ANS 6.1 .11991 standard.

$\begin{array}{llll}\text { print } & 10 & 110 & 120\end{array}$

nps 1.005

CSCOBAP.INP Storage Can, $3.6 \mathrm{~kg} \mathrm{Pu}$, gamma ray calculation

PHOTON Dose Rate Calcs for calciner - CSCO8AP

c Hi 240Pu PUO2 Storage Can Photon Source is in Cell 1 $N$ Shield Lucite $-1.185 \mathrm{~g} / \mathrm{cm} 3-4$ inches top, 5 inches bottom Lower Shields are $1 / 8$ inch of stainless Steel (both sides) Upper Shields are $3 / 4$ inch of Glass (no Boron)(both sides)

Full Room Model with Concrete Walls, Roof, and Metal Partitions

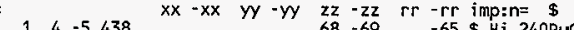

$14-5.438$

$\begin{array}{lll}2 & 9 & -7.84 \\ 3 & 9 & -7.84\end{array}$

$68-69$

$-65 \$ \mathrm{Hi} 240 \mathrm{PuO} 2$

$\begin{array}{lll}3 & 9 & -7.84 \\ 4 & 8 & -11.34\end{array}$

\begin{tabular}{llll}
9 & -68 & -65 & $\$$ Can SS Bot \\
\hline & 65 & -66 & $\$$ Can SS Watl
\end{tabular}

$\begin{array}{llllllll}4 & 8 & -11.34 & 9 & -69 & 66 & -67 & \$\end{array}$ Can Lead Shield

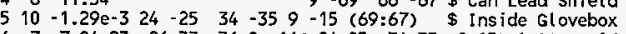

$6 \quad 7 \quad-7.8423-26 \quad 33-36 \quad 8 \quad-16(-24: 25:-34: 35:-9: 15)$ \$ SS Inside

$78-11.3422-27 \quad 32-37 \quad 7 \quad-17(-23: 26:-33: 36:-8: 16)$ \$ Lead Shield

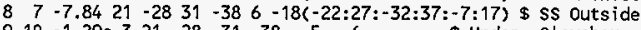

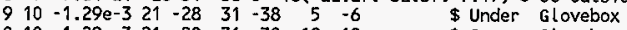

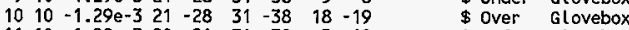

$\begin{array}{lllllllll}11 & 10 & -1.29 e-3 & 20 & -21 & 31 & -38 & 5 & -19\end{array}$

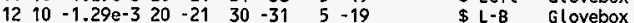

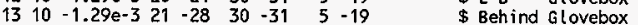


$14 \quad 10-1.29 \mathrm{e}-3 \quad 28-29 \quad 30-31$

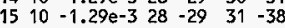

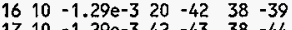

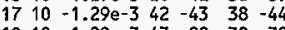

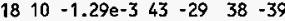

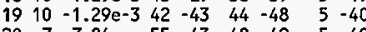
$\begin{array}{lllllllll}20 & 7 & -7.84 & 55 & -43 & 48 & -49 & 5 & -40\end{array}$ $2114-1.185 \quad 52-43 \quad 49-50$ $\begin{array}{lllllllll}22 & 7 & -7.84 & 53 & -43 & 50 & -51 & 5 & -40\end{array}$

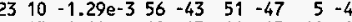
$\begin{array}{lllllllll}24 & 15 & -2.14 & 42 & -43 & 44 & -45 & 40 & -41\end{array}$ $\begin{array}{lllllllll}25 & 14 & -1.185 & 52 & -43 & 45 & -46 & 40 & -4\end{array}$ $\begin{array}{lllllllll}26 & 15 & -2.14 & 53 & -43 & 46 & -47 & 40 & -41\end{array}$

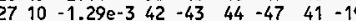
$2810-1.29 e-3 \quad 42-53 \quad 48-39$ $\begin{array}{lllllllll}29 & 7 & -7.84 & 55 & -52 & 49 & -39 & 5 & -40\end{array}$

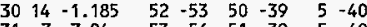

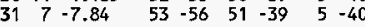
$\begin{array}{lllllllll}32 & 10 & -1.29 e-3 & 56 & -54 & 47 & -39 & 5 & -40\end{array}$

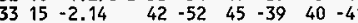
$\begin{array}{lllllllll}34 & 14 & -1.185 & 52 & -53 & 46 & -39 & 40 & -41\end{array}$

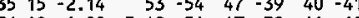
$\begin{array}{lllllllll}36 & 10 & -1.29 \mathrm{e}-3 & 42 & -54 & 47 & -39 & 41 & -19\end{array}$

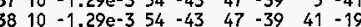

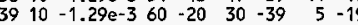

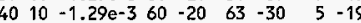

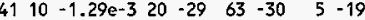

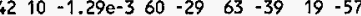
$\begin{array}{lllllllll}43 & 7 & -7.84 & 60 & -29 & 62 & -63 & 5 & -57\end{array}$

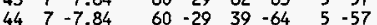

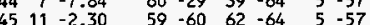
$20-61 \quad 62-64 \quad 5-57$ $\begin{array}{llllll}29 & -61 & 62 & -64 & 5 & -57\end{array}$

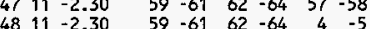
48 11 -2.30

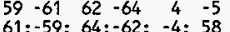

\begin{tabular}{|c|c|}
\hline 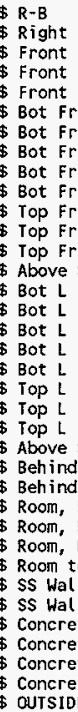 & 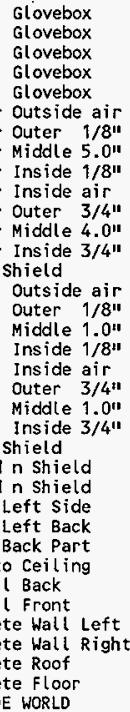 \\
\hline
\end{tabular}

Concrete Floor

FEED TANK O.R. - $\left(5 / 16^{ \pm 1}-0.040^{\circ \prime}\right)$

$\$$ FEED TANK O.R.

$\$$ FEED TANK FLANGE O.R.

$\$$ CONCRETE FLOOR bottom (24" thick)

$\$$ CONCRETE FLOOR SUIface

$\$$ GLOVEBOX bottom surface

\$ GLOVEBOX Stainless steel (3/32"1)

$\$$ GLOVEBOX Lead Shield (3/16")

$\$$ GLOVEBOX Stainless Steel (3/32")

$\$$ FEED TANK Bottom Flange bottom

FEED TAKK Bottom Flange botton

$\$$ FEED TANK solution level (10 L)

$\$$ FEED TANK TOp Flange bottom

$\$$ FEED TANK TOP Flange top

$\$$ GLOVEBOX top inside surface

$\$$ GLOVEBOX stainless steel (3/32")

$\$$ GLOVEBOX Lead Shield (3/16")

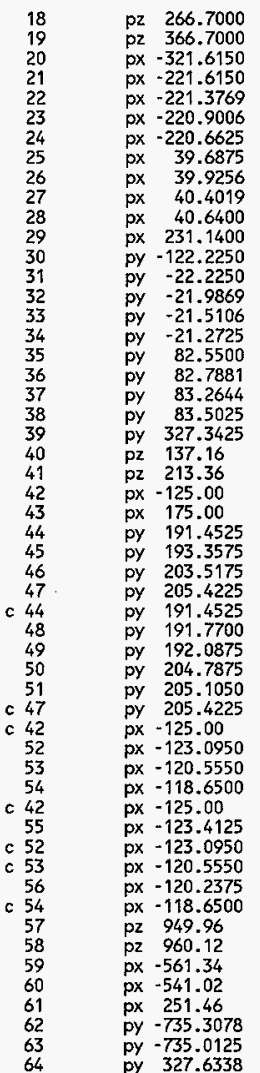

$\$$ GLOVEBOX Stainless steel (3/32')

$\$$ MODEL TOP (GB rOof +100 CII)

$\$$ MODEL LEFT SIDE (GB side $+100 \mathrm{~cm}$ )

\$ GLOVEBOX left surface

$\$$ GLOVEBOX Stainless steel (3/32")

$\$$ GLOVEBOX Lead Shield (3/16")

\$ GLOVEBOX stainless steel (3/32")

$\$$ GLOVEBOX right inside surface

\$ GLOVEBOX stainless steel (3/32")

\$ GLOVEBOX Lead Shield (3/16")

\$ GLOVEBOX Stainless steel (3/32")

$\$$ MODEL RIGHT SIDE (GB side +75")

$\$$ MODEL BACK SIDE (GB back $+100 \mathrm{~cm}$ )

$\$$ GLOVEBOX back surface

$\$$ GLOVEBOX Stainless Steel (3/32")

$\$$ GLOVEBOX Lead Shield (3/16:1)

\$ GLOVEBOX stainless steel (3/32")

$\$$ GLOVEBOX front inside surface

$\$$ GLOVEBOX stainless steel $\left(3 / 32^{* 1}\right)$

GLOVEBOX Lead Shield (3/16")

\$ GLOVEBOX Stainless Steel (3/3211)

$\$$ MODEL FRONI SIDE (GB front +96")

$\$ 4.5$ ' High $n$ shield

+2.5' top n shield

$\$$ Left End $n$ Shield

$\$$ Right End n Shield

$\$ 3 / 4 "$ n Shield Material outside

$\$ 4.0 "$ n Shield Material outside

$\$ 3 / 4 "$ "n Shield Material outside

Operator side n shield

$\$ 3 / 4 "$ n Shield Material outside

1/8" n Shield Material outside

5 Tu" nhield Material outside

$\$$.an Shield Material outside

$\$ 1 / 8^{\prime \prime}$ n Shield Material outside

\$1/8" n Shield Material ine

$\$$ Operator side $n$ Shield

$\$ 3 / 4 "$ n Shield Material outside

$\$ 1.0 "$ n shield Material outside

$\$ 3 / 4 "$ n shield Material outside

$\$$ Operator Side $\mathrm{n}$ Shield

$\$ 3 / 4^{\prime \prime} n$ Shield Material outside

$\$ 1 / 8^{\prime \prime} n$ Shield Material outside

\$1.0" n shield Material outside

$\$ 3 / 4 "$ " n shield Material outside

1/8"n Shield Material outside

$\$ 1 / 8^{12}$ n Shield Materia

$\$$ Operator side $n$ Shield

$\$ 4$ inch concrete roof [H-2-16174]

$\$ 8$ inch concrete wall left side

$\$$ Left Wall Surface [H-2-16140]

$\$ 8$ inch concrete wall right side

$\$ 18$ Gauge Metal $\left(.0516^{\prime \prime *}(4.0 / 1.8) \Rightarrow .2913 \mathrm{~cm}\right)$

$\$$ Back Wall Surface [H-2-16140]

18 Gauge Metal $(.051611 *(4,0 / 1.8)=2913 \mathrm{~cm}$ ) 

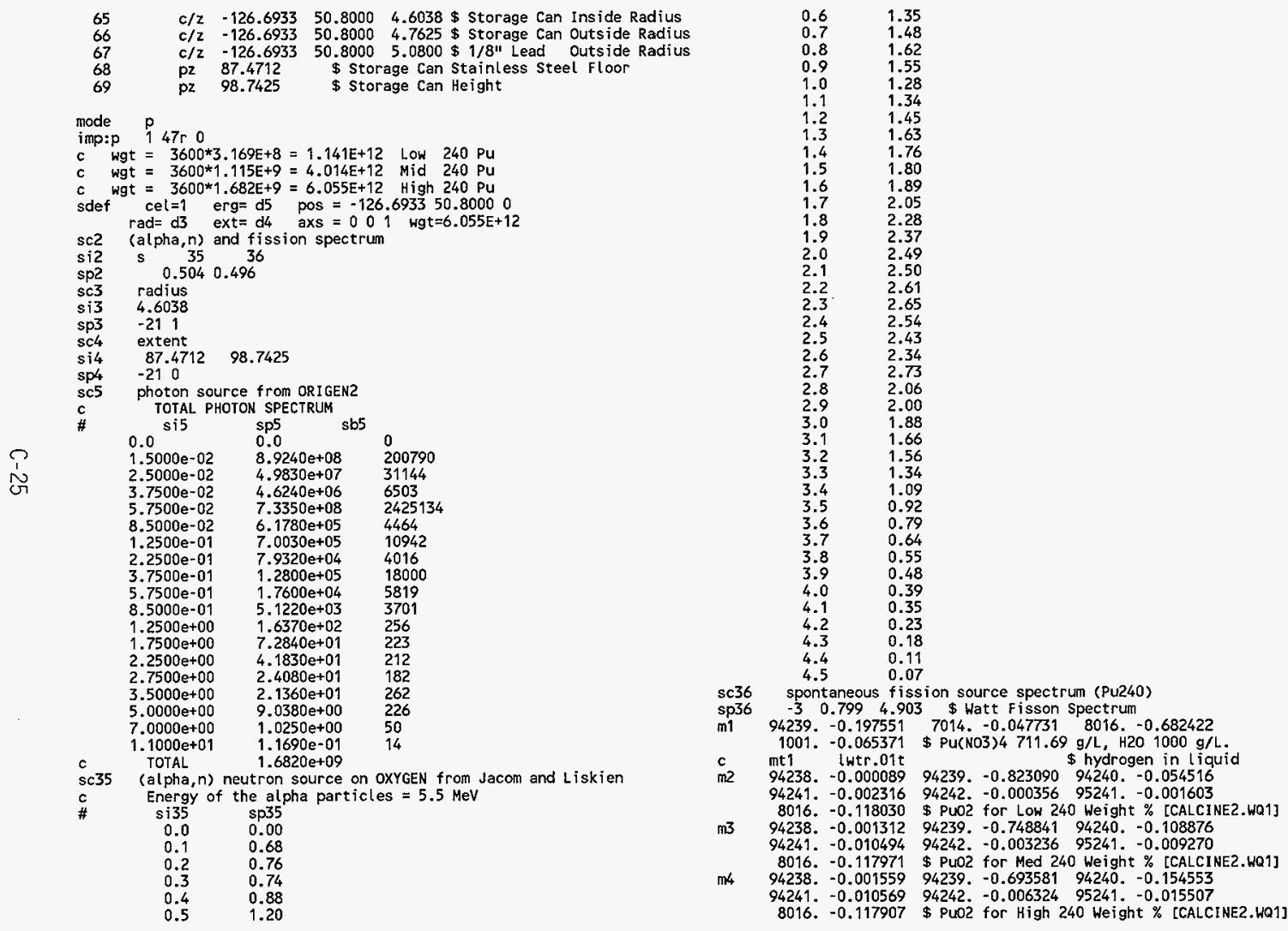

$\operatorname{sc} 36$ spon

sp36 -3 0.7994 .903 S Watt Fisson Spectrum

m1 94239, $-0.197551 \quad 7014,-0.047731 \quad 8016,0.682422$

c mt 1. lwtr.01t Pu(N03)4 $711.69 \mathrm{~g} / \mathrm{L}$.

m2 $94238-0.00008994239-0.823090 \quad 94240-0.054516$

$94241-0.00231694242-0.00035695241-0.001603$
$\mathrm{m3}$
TINE2.WQ13

$94241 .-0.010494$ 9424. 0.7403236 95241-0. 009270

nh $94238 .-0.001559$ 94239. -0.693581 94240. -0.15453

8016. $-0.117907 \$$ PUOZ for High 240 Weight \% [CALCINE2. HQ1] 
14000. -0.376753 5010. -0.007986 5011. -0.032386

11023. -0.029674 13027. -0.012173 19000. -0.000830

8016. $-0.540198 \$ 7740$ PYREX TYPe I Glass [CALCINE1.WQ1]

mo 26000. -0.74 24000. -0.18 28000: $-0.08 \$$ SS 304L Flanges

m7 26000. -0.98 28000. $-0.02 \$$ stainless steel Glovebox

m8 82000. -1.00 \$ Lead Glovebox Shield

$m 926000$. -1 \$ 310 S

m10 7014. -.78084 8016. $-0.20946 \quad 18000.35 \mathrm{c}-0.00934$

1001. - .00036 \$ Air

c mt10 Iwtr.01t \$ hydrogen in liquid, gas

m11 13027. -.034 20000. -0.044 26000. -0.014

1001. - .001 8016. -0.532 14000. -0.337

11023. -.029 \$ Concrete (common Portland) ARH-600 II.F.1-3

$\begin{array}{lllll}c & m+11 & \text { poly. 01t } & & \text { \$ hydrogen in sol id } \\ \mathrm{m} 12 & 6000 & -0.5925 & 5010 .-0.05905011 . & -0.2410 \text { \$ Neutron }\end{array}$

1001. -0.0854 14000. -0.0101 8016. $-0.0076 \$$ shield

26000. -0.0038 13027. -0.000425055 . $-0.0002 \$ 210$

c mt12 poly.01t grph.01t \$ hydrogen, carbon in solid (1.19

$\mathrm{g} / \mathrm{cm} 3$ ) $8016 .-0.3649 \quad 6000,-0.3439$ 13027, $-0.1906 \$$ Neutron

1001. $-0.078420000=0.0123$ 14000:-0.0009 \$ Shield

5010. -0.00138 5011. -0.00562 12000. $-0.0006 \$ 207$

$11023-0.00063011 .-0.0056212000 .-0.0006 \$ 207$

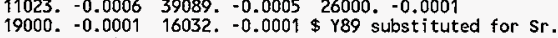
$\mathrm{g} / \mathrm{cm} 3)^{\mathrm{mt13}}$ poly.01t grph.01t \$ hydrogen, carbon in solid (1.4

m14 6000. -0.599848 1001. -0.080538 8016. -0.319614 \$ Lucite

c mt14 poly.01t grph.01t \$hydrogen in solid $(1.185 \mathrm{~g} / \mathrm{cm} 3)$

Lucite

m15 14000. $-0.392603 \$ 7740$ PYREX Type I Glass [no Boron, $2.14 \mathrm{~g} / \mathrm{cm} 3$ ] 11023. -0.030923 13027. -0.012685 19000. -0.000865

8016. $-0.562924 \$ 7740$ PYREX Type I Glass [CALCINE1.WQ1]

m16 6000. -0.755751 1001. $-0.0554918016 .-0.188758$ \$ Lexan

$c$ mt16 poly.01t grph.01t \$hydrogen in solid $(1.2 \mathrm{~g} / \mathrm{cm} 3)$

Lexan

Photon dose conversion factors from ANS 6.1.1 1991

de0 $\quad \log \begin{array}{llllll}.01 & .015 & .02 & .03 & .04 & .05\end{array}$

$\begin{array}{llllll}.06 & .08 & .010 & .15 & .02 & .30 \\ .40 & .50 & .60 & .80 & 1.0 & 1.5\end{array}$

$\begin{array}{cccccc}.40 & 3.0 & .00 & .80 & 1.0 & 1.5 \\ 2.0 & 3.0 & 4.0 & 5.0 & 6.0 & 8.0\end{array}$

$10.0 \quad 12.0$

dfo $\quad \log 2.232 \mathrm{e}-5 \quad 5.652 \mathrm{e}-5 \quad 8.568 \mathrm{e}-5 \quad 1.184 \mathrm{e}-4 \quad 1.314 \mathrm{e}-4 \quad 1.382 \mathrm{e}-4$

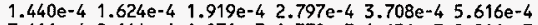

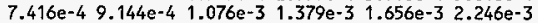
$2.758 \mathrm{e}-3 \quad 3.672 \mathrm{e}-3 \quad 4.500 \mathrm{e}-3 \quad 5.292 \mathrm{e}-3 \quad 6.012 \mathrm{e}-3 \quad 7.488 \mathrm{e}-3$ $2.758 \mathrm{e}-3$
$8.892 \mathrm{e}-3$
$1.040 \mathrm{e}-2$

fc4 Photon Dose behind Neutron Shield, Right Side Leak Around

f4:p 37 \$ Operator behind Neutron Shield

cf4 18 \$Flagging Cells for leak-through,

skyshine

$f 14: \mathrm{p} 37$ \$ Operator behind Neutron Shield

cf14 23

skyshine fc24 Photon Dose behind Neutron Shield, Front Upper $n$ Shield Leak Through f24:p 37 \$ Operator behind Neutron Shield

cf24 $26 \quad$ Flagging Cells for leak-through,

skyshine

fc34 Photon Dose behind Neutron Shield, Left Lower $n$ Shield Leak Through

$\begin{array}{lll}\text { f34:p } 37 & 32 & \$ \text { Operator behind Neutron Shield } \\ \text { cf34 } & \$ \text { Flagging Cells for leak-through, }\end{array}$

skyshine

S Flagging Cells for leak-through,

fc44 Photon Dose behind Neutron Shield, Left Upper $n$ Shield Leak Through

f44:p 37 Operator behind Neutron shield

cf44 35 \$ Flagging Cells for leak-through,

skyshine

fc54 Photon Dose behind Neutron Shield, Roof Reflection

f54:p $37 \quad$ \$Operator behind Neutron Shield

cf54 $38 \quad \$ \mathrm{Flagging}$ Cells for leak-through,

skyshine

fc64 Photon Dose behind Neutron Shield, Steel Wall Back Reflection

f64:p 37 \$ Operator behind Neutron Shield

cf64 43 \$ Flagging Cells for leak-through,

skyshine

fc74 Photon Dose behind Neutron Shield, Concrete Floor Effect

f74:p $37 \quad \$$ Operator behind Neutron Shield

cf 74

48 \$ Flagging Cells for leak-through,

skyshine

fc84 Photon Dose behind Neutron shield, sum of All Cells fLAGGING!!

f84:p $37 \quad$ \$Operator behind Neutron Shield

$\begin{array}{llllllllll}\text { cf84 } & 18 & 23 & 26 & 32 & 35 & 38 & 43 & 48 & \$\end{array}$ Flagging Cells

fc5 Photon dose at $30 \mathrm{~cm}$ from outside surface of 6 in. pipe

c Total Dist from outside $=30.0+50.80+5.08=85.88 \mathrm{~cm}$

$f 5: p \quad-126.6933 \quad 85.88 \quad 93.1068 \quad 0$

fc15 Photon dose at $30 \mathrm{~cm}$ from outside surface of Glovebox

c Total Dist from outside $=30.0+83.5025=113.5025 \mathrm{~cm}$

f15: $\mathrm{p} \quad-126.6933 \quad 113.502593 .1068 \quad 0$

fc25 Photon dose at 5 feet from outside surface of Glovebox

c Total Dist from outside $=152.8+83.5025=235.9025 \mathrm{~cm}$

f25:p $\quad-126.6933235 .902593 .1068 \quad 0$

fc35 Photon dose at 6 in. in front of lower $n$ Shield

c Total Dist from outside $=191.4525-6^{\star} 2.54=176.2125 \mathrm{~cm}$

435:p $0176.2125 \quad 91.44 \quad 0$

fc45 Photon dose in middle of outer 1/8" SST $n$ Shield

c Total Dist from outside $=(191.7700+192.0875) / 2=191.9288 \mathrm{~cm}$ f45:p $0191.9288 \quad 91.44 \quad 0$

fc55 Photon dose in middle of 5.0 " lower $n$ shield

c Total Dist from outside $=(192.0875+204.7875) / 2=198.4375 \mathrm{~cm}$

f55: $\mathrm{P} \quad 0198.4375 \quad 91.44 \quad 0$

fc65 Photon dose in middle of inner 1/8" stainless steel $n$ shield

$c$ Total Dist from outside $=(204.7875+205.1050) / 2=204.9462 \mathrm{~cm}$ f65:p $0204.9462 \quad 91.44 \quad 0$

fc75 Photon dose at 6 in. behind inside lower $n$ shield

c Total D ist from outside $=205.4225+6 * 2.54=220.6225 \mathrm{~cm}$ $0220.662591 .44 \quad 0$

fc85 Photon dose at 6 in. in front of upper $n$ shield

c Total Dist from outside $=191.4525-6 * 2.54=176.2125 \mathrm{~cm}$ 
fc95 Photon dose in middle of outer 3/4" glass $n$ Shield

c Total Dist from outside $=(191.4525+193.3575) / 2=192.4050 \mathrm{~cm}$ f95:p $\quad 0192.4050182 .88 \quad 0$

fe105 Photon dose in middle of 4.0" upper $n$ shield

c Total Dist from outside $=(193.3575+203.5175) / 2=198.4375 \mathrm{~cm}$ f105:p $0198.4375182 .88 \quad 0$

fc115 Photon dose in middle of inner $3 / 4^{\prime \prime}$ glass $n$ shield

c Total Dist from outside $=(203.5175+205.4225) / 2=204.4700 \mathrm{~cm}$

f115:p $0204.4700 \quad 182.88$ o

f115: $p$ photon dose at 6 in behind inside upper $n$ shield

fc125 Photon dose at 6 in. behind inside upper n Shield

f125:p $0220.6625 \quad 182.88 \quad 0$

print $10 \quad 110 \quad 120$

ctme 180.0

nps $1.0 \mathrm{e} 7$ 
HNF-SD-CP-TI-218 Rev. 0

This page intentionally left blank 
HNF-SD-CP-TI-218 Rev. 0

APPENDIX D

HAND DOSE RATE MCNP INPUT FILES

CRCH02NP. INP $3.6 \mathrm{~kg}$ Pu Receipt Can with Hands, neutron and capture gammas

CRCH02P. INP $3.6 \mathrm{~kg}$ Pu Receipt Can with Hands, gamma ray calculation 
HNF-SD-CP-TI-218 Rev. 0

This page intentionally left blank 
CRCH02NP.INP $3.6 \mathrm{~kg}$ Pu Receipt Can with Hands, neutron and capture garmas NEUTRON Dose Rate Calcs for calciner - CRCHO2NP.INP

c Hi 24OPu PuO2 Receipt Can Neutron Source is in Cell 1

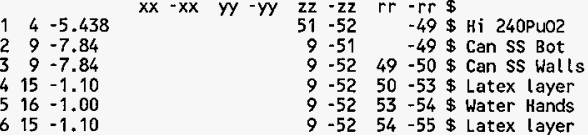

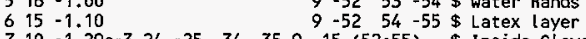

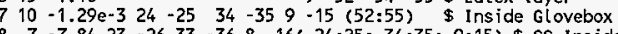

$8 \quad 7-7.8423-2633-36 \quad 8-16(-24: 25:-34: 35:-9: 15)$ \$ SS Inside

$98-11.3422-2732-37 \quad 7-17(-23: 26:-33: 36:-8: 16)$ \$ Lead Shield

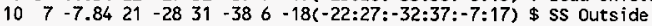

$11 \quad 10-1.29 \mathrm{e}-3 \quad 21-28 \quad 31-38 \quad 5 \quad-6 \quad$ U Under Glovebox

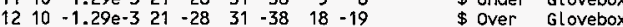

$1310-1.29 \mathrm{e}-3 \quad 20-21 \quad 31-38 \quad 5-19 \quad$ \$ Left Glovebox

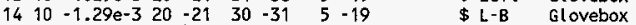

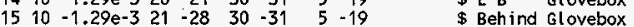

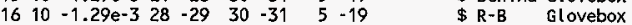

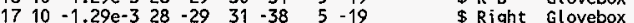

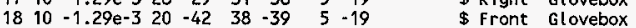

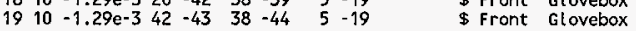

$2010-1.29 e-3 \quad 43-29 \quad 38-39 \quad 5-19 \quad$ \$ Front Glovebox

$2110-1.29 \mathrm{e}-3 \quad 42-43 \quad 44-45 \quad 5-40 \quad$ \$ Bot Outer 3/4"1

$2210-1.29 \mathrm{e}-3 \quad 42-43 \quad 45-46 \quad 5 \quad-40 \quad$ \$ Bot Middle $1.0^{\prime \prime}$

$2310-1.29 \mathrm{e}-3 \quad 42-43 \quad 46-47 \quad 5 \quad-40 \quad$ \$ Bot Inner 3/4"

$24 \quad 10-1.29 \mathrm{e}-3 \quad 42-43 \quad 47 \quad-48 \quad 5 \quad-40 \quad$ \$ Bot Inside $1 / 41$

$2510-1.29 e-3 \quad 42-43 \quad 44-45 \quad 40-41 \quad$ \$ Top Outer $3 / 41$

$26 \quad 10-1.29 \mathrm{e}-3 \quad 42-43 \quad 45-46 \quad 40-41 \quad$ \$ Top Middle 1.011

$2710-1.29 \mathrm{e}-3 \quad 42-43 \quad 46-47 \quad 40-41 \quad$ \$ Top Inner $3 / 4$

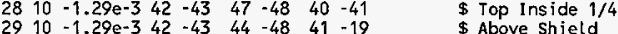

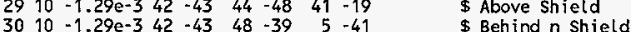

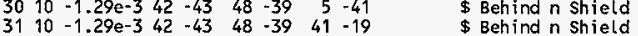

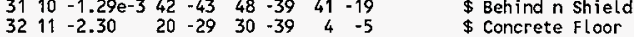

330 29:-20: 39:-30: $-4: 19$ \$ OUTSIDE WORLD

$\begin{array}{rrr}1 & \mathrm{cz} & 7.7610 \\ 2 & \mathrm{cz} & 8.4534 \\ 3 & \mathrm{cz} & 14.2875 \\ 4 & \mathrm{pz} & -60.9600 \\ 5 & \mathrm{pz} & 0.0000 \\ 6 & \mathrm{pz} & 86.3600 \\ 7 & \mathrm{pz} & 86.5981 \\ 8 & \mathrm{pz} & 87.0744 \\ 8 & \mathrm{pz} & 87.3125 \\ 9 & \mathrm{pz} & 171.4500 \\ 10 & \mathrm{pz} & 171.7200 \\ 11 & \mathrm{pz} & 1700 \\ 12 & \mathrm{pz} & 224.5663 \\ 13 & \mathrm{pz} & 233.6800\end{array}$

$\$$ FEED TANK O.R. - (5/16"1-0.040"1)

$\$$ FEED TANK O.R.

$\$$ FEED TANK FLANGE O.R.

$\$$ CONCRETE FLOOR bottom (24" thick)

$\$$ CONCRETE FLOOR surface

$\$$ GLOVEBOX bottom surface

\$ GLOVEBOX Stainless Steel (3/32")

$\$$ GLOVEBOX Lead Shield (3/16")

$\$$ GLOVEBOX Stainless steel ( $\left.3 / 32^{\prime \prime}\right)$

$\$$ FEED TANK Bottom flange bottom

$\$$ FEED TANK Bottom $\mathrm{Flange}$ top

$\$$ FEED TANK Solution level $(10 \mathrm{~L})$ $\begin{array}{lll}\text { pz } & 234.9500 & \text { \$ FEED TANK TOP Flange top } \\ \text { pz } & 265.7475 & \text { \$ GLOVEBOX top inside surface }\end{array}$

pz 265.9856

pz 266.7000

$\mathrm{pz} 366.7000$

$\mathrm{px}-321.6150$

px -221.6150

$\mathrm{px}-221.3769$

$\mathrm{px}-220.9006$

$\mathrm{px}-220.6625$

$\mathrm{px} \quad 39.6875$

$\mathrm{pX} \quad 39.9256$

$\mathrm{px} \quad 40.6400$

px 231.1400

py -122.2250

py -22.2250

py -21.9869

py -21.5106

$\begin{array}{ll}\text { py } & -21.568 \\ \text { py } & -21.2725\end{array}$

py 82.5500

py 82.788

Py 83.2644

py 83.5025

py 327.3425

pz 152.40

pz 213.36

px -125.00

px 175.00

py 198.4375

py 200.3425

py 202.8825

py 204.7875

py 205.4225

$c / 2 \quad-168.5925$

$c / 2 \quad-168.5925$

pz 87.4712

$c / 2 \quad-168.5925$

$c / 2 \quad-168.5925$

\$LLOVEBOX Stainless steel (3/32")

$\$$ GLOVEBOX Lead Shield (3/16")

$\$$ GLOVEBOX Stainless steel (3/32")

$\$$ MODEL TOP (GB rOof $+100 \mathrm{~cm}$ )

$\$$ MODEL LEFT SIDE (GB side $+100 \mathrm{~cm}$ )

$\$$ GLOVEBOX left surface

GLOVEBOX Stainless Steel (3/32")

$\$$ GLOVEBOX Lead Shield (3/16")

$\$$ GLOVEBOX stainless steel (3/32")

$\$$ GLOVEBOX right inside surface

$\$$ GLOVEBOX Stainless steel (3/32")

$\$$ GLOVEBOX Lead Shield (3/16")

\$ GLOVEBOX Stainless Steel (3/32"1)

\$ MODEL RIGHT SIDE (GB side $+75^{\prime \prime}$ )
$\$$ MODEL BACK SIDE (GB back $+100 \mathrm{~cm}$ )

$\$$ GLOVEBOX back surface

$\$$ GLOVEBOX Stainless Steel (3/32"1)

$\$$ GLOVEBOX Stainless Steel (3/32")

\$ GLOVEBOX Stainless Steel (3/32")

\$ GLOVEBOX Stainless Steel (3/32")

\$ GLOVEBOX front inside surface

\$ GLOVEBOX Stainless Steel (3/3

\$ GLOVEBOX Lead Shield (3/16")

$\$$ GLOVEBOX Stainless Steel (3/32")

$\$ 5$, High $n$ shield

$\$+2$ ' Top $n$ shield

$\$$ Left End $n$ Shield

$\$$ Right End $n$ Shield

$\$ 3 / 4 "$ n Shield Materia

$\$$ 1.0" n Sh ield Material

1/4" n shield Material

i/4"n shield Material

$\$$ Operator side $n$ shiel

$29.8450 \quad 4.6038$ \$ Receipt Can Inside Radius

$29.8450 \quad 4.7625$ \$ Receipt Can Outside Radius

$\$$ Receipt Can Stainless Steel Floor

Receipt Can Height

29.84507 .5406 Water Hand Outside Radius

29.84507 .7788 \$ Latex Glove Outside Radius

mode $n \mathrm{p}$

imp:n $131 \% 0$

imp:p $131 r$ r

$=378000$ Low $240 \mathrm{Pu}$

c wgt $=3600 * 215=774000 \mathrm{Mid} 240 \mathrm{Pu}$

$c$ wgt $=3600 * 295 \quad=1062000 \mathrm{High} 240 \mathrm{Pu}$

sdef cel $=1$ erg= d2 pos $=-168.592529 .84500$

sc2 (alpha, $n$ ) and fission spectrum 


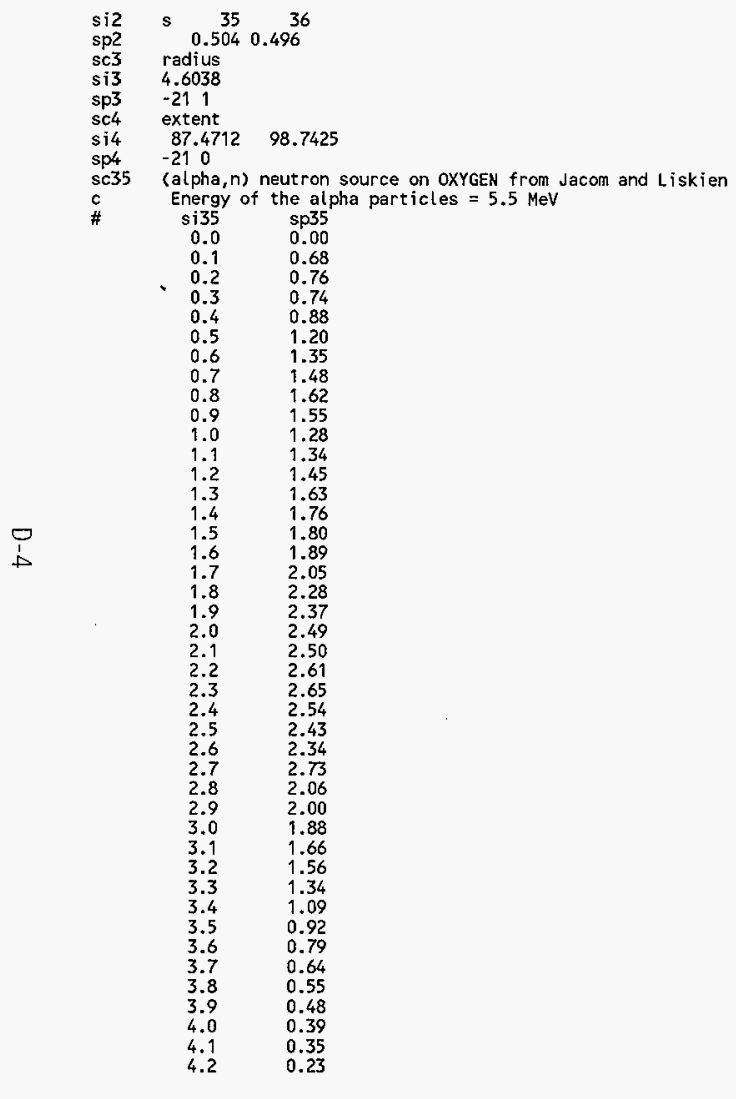

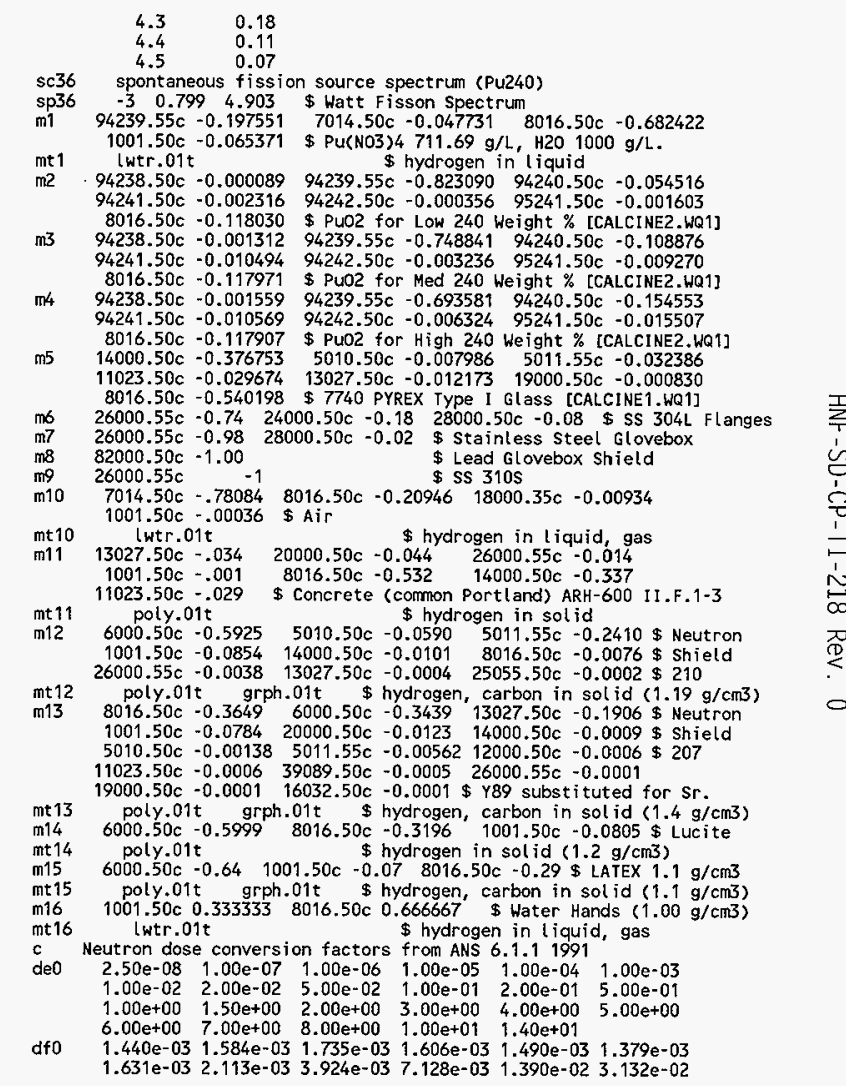


$5.148 \mathrm{e}-02 \quad 6.588 \mathrm{e}-02 \quad 7.704 \mathrm{e}-02 \quad 9.504 \mathrm{e}-02 \quad 1.080 \mathrm{e}-01 \quad 1.177 \mathrm{e}-01$ $1.249 \mathrm{e}-01 \quad 1.314 \mathrm{e}-01 \quad 1.368 \mathrm{e}-01 \quad 1.476 \mathrm{e}-01 \quad 1.728 \mathrm{e}-01$ Neutron dose conversion factors from ANS 6.1 .11977

de4 $2.5 \mathrm{e}-08$ 1.0e-07 $1.0 \mathrm{e}-06 \quad 1.0 \mathrm{e}-05 \quad 1.0 \mathrm{e}-04 \quad 1.0 \mathrm{e}-03$

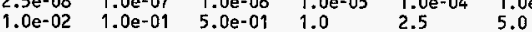

$\begin{array}{llll}7.0 & 10.0 & 14.0 & 20.0\end{array}$

5.0

df4 $\quad 3.670 \mathrm{e}-03 \quad 3.670 \mathrm{e}-03 \quad 4.460 \mathrm{e}-03 \quad 4.540 \mathrm{e}-03 \quad 4.180 \mathrm{e}-03 \quad 3.760 \mathrm{e}-03$ $3.560 \mathrm{e}-032.170 \mathrm{e}-02 \quad 9.260 \mathrm{e}-02 \quad 1.320 \mathrm{e}-01 \quad 1.250 \mathrm{e}-01 \quad 1.560 \mathrm{e}-0$

$1.470 \mathrm{e}-011.470 \mathrm{e}-012.080 \mathrm{e}-012.270 \mathrm{e}-01$

$\mathrm{fc} 4$ dose in hands modeled by water 1 inch thick (Cel! 5) $\mathrm{f} 4: \mathrm{n} \quad 5$

c Photon dose conversion factors from ANS 6.1.1 1977

$\begin{array}{rlllll}\text { de14 } \log 0.01 & 0.03 & 0.05 & 0.07 & 0.10 & 0.15 \\ 0.20 & 0.25 & 0.30 & 0.35 & 0.40 & 0.45 \\ 0.50 & 0.55 & 0.60 & 0.65 & 0.70 & 0.80 \\ 1.00 & 1.40 & 1.80 & 2.20 & 2.60 & 2.80 \\ 3.25 & 3.75 & 4.25 & 4.75 & 5.00 & 5.25\end{array}$

$\begin{array}{llllll}3.25 & 3.75 & 4.25 & 4.75 & 5.00 & 5.25\end{array}$

$\begin{array}{llllll}5.75 & 6.25 & 6.75 & 7.50 & 9.00 & 11.00\end{array}$

df14 $\log 3.960 \mathrm{e}-03 \quad 5.820 \mathrm{e}-04 \quad 2.900 \mathrm{e}-04 \quad 2.580 \mathrm{e}-04 \quad 2.830 \mathrm{e}-04 \quad 3.790 \mathrm{e}-04$ $5.010 \mathrm{e}-04 \quad 6.310 \mathrm{e}-04 \quad 7.590 \mathrm{e}-04 \quad 8.780 \mathrm{e}-04 \quad 9.850 \mathrm{e}-04 \quad 1.080 \mathrm{e}-03$ $1.170 \mathrm{e}-03 \quad 1.270 \mathrm{e}-03 \quad 1.360 \mathrm{e}-03 \quad 1.440 \mathrm{e}-03 \quad 1.520 \mathrm{e}-03 \quad 1.680 \mathrm{e}-03$

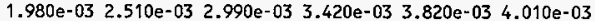
$4.410 \mathrm{e}-03 \quad 4.830 \mathrm{e}-035.230 \mathrm{e}-035.600 \mathrm{e}-035.800 \mathrm{e}-03 \quad 6.010 \mathrm{e}-03$ $6.370 \mathrm{e}-03 \quad 6.740 \mathrm{e}-03 \quad 7.110 \mathrm{e}-03 \quad 7.660 \mathrm{e}-03 \quad 8.770 \mathrm{e}-03 \quad 1.030 \mathrm{e}-02$ $\begin{array}{ll}6.370 \mathrm{e}-03 & 6.740 \mathrm{e}-03 \\ 1.180 \mathrm{e}-02 & 1.330 \mathrm{e}-02\end{array}$

\begin{abstract}
fc14 dose in hands modeled by water 1 inch thick (Cell 5)
\end{abstract}
f14:p 5

fc15 dose at $30 \mathrm{~cm}$ from outside surface of Receipt can

c Y Dist $=30.0+4.6038+29.8450=64.4488 \mathrm{~cm}$

f15:n $\quad-168.5925 \quad 64.4488 \quad 93.10685 \quad 0$

$\mathrm{fm} 152.0 \quad \$$ Factor of 2 times the ANS 6.1 .11991 standard. $\mathrm{fc} 25$ dose at $30 \mathrm{~cm}$ from outs ide surface of Glovebox

c $Y$ Dist $=30.0+83.5025=113.5025 \mathrm{~cm}$

$\begin{array}{lllll}f 25: n & -168.5925 & 113.5025 & 93.10685 & 0\end{array}$ fc35 dose at 5 feet from outside surface of Glovebox

c Y Dist $=152.8+83.5025=235.9025 \mathrm{~cm}$

f35:n $\quad-168.5925 \quad 235.9025 \quad 93.10685 \quad 0$

fm35 2.0 \$ Factor of 2 times the ANS 6.1.1 1991 Standard.

fc45 dose at 6 in. in front of lower $n$ Shield

c Total Dist from outside $=198.4375-6^{\star} 2.54=183.1975 \mathrm{~cm}$

f45:n $0183.1975135 .0 \quad 0$

fM4 2.0 \$ Factor of 2 times the ANS 6.1.1 1991 standard.

$\mathrm{fc55}$ dose in middle of outer 3/4" PYREX $n$ shield

c Total Dist from outside $=(198.4375+200.3425) / 2=199.3900 \mathrm{~cm}$ f55:n $0199.3900135 .0 \quad 0$

fm55 2.0 Factor of 2 times the ANS 6.1 .11991 standard. fc65 dose in middle of $1.0^{\prime \prime} \mathrm{n}$ shield

$c$ Iotal Dist from outside $=(200.3425+202.8825) / 2=201.6125 \mathrm{~cm}$ f65:n Total Dist from outside

fm65 2.0 \$ Factor of 2 times the ANS 6.1 .11991 standard.

$f c 75$ dose in middle of inner 3/4" PYREX $n$ Shield

$c$ Total Dist from outside $=(202.8825+204.7875) / 2=203.8350 \mathrm{~cm}$ f75:n $\quad 0203.8350135 .0 \quad 0$

fm75 $2.0 \quad \$$ Factor of 2 times the ANS 6.1 .11991 standard. fc85 dose in middle of inside $1 / 4$ " $n$ shield

$c$ Total Dist from outside $=(204.7875+205.4225) / 2=205.1050 \mathrm{~cm}$ f85:n $0205.1050135 .0 \quad 0$

fm85 2.0 \$ Factor of 2 times the ANS 6.1.1 1991 standard. fc95 dose at 6 in. behind inside $n$ shield

c Total Dist from outside $=205.4225+6 * 2.54=220.6225 \mathrm{~cm}$

f95:n $0220.6625 \quad 135.0 \quad 0$

fm95 $2.0 \quad \$$ Factor of 2 times the ANS 6.1 .11991 standard

fc105 dose at 6 in. in front of upper n shield

c Total Dist from outside $=198.4375-6 * 2.54=183.1975 \mathrm{~cm}$

f105:n $\quad 0$ 183.1975 200.0

$\mathrm{fm} 1052.0 \quad \$$ Factor of 2 times the ANS 6.1 .11991 standard. fc115 dose in middle of outer 3/4" PYREX $n$ shield

c Total Dist from outside $=(198.4375+200.3425) / 2=199.3900 \mathrm{~cm}$ f115:n $0199.3900200 .0 \quad 0$

fm115 2.0 \$ Factor of 2 times the ANS 6.1 .11991 standard. fc125 dose in middle of $1.0^{\prime \prime} \mathrm{n}$ shield

c Total Dist from outside $=(200.3425+202.8825) / 2=201.6125 \mathrm{~cm}$ f125:n 0201.6125200 .00

$\mathrm{fm} 1252.0 \quad \$$ Factor of 2 times the ANS 6.1 .11991 standard. fc135 dose in middle of inner $3 / 4 "$ PYREX $n$ Shield

c Total Dist from outside $=(202.8825+204.7875) / 2=203.8350 \mathrm{cn}$

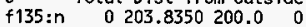

f 1352.0 \$ Factor of 2 times the ANS 6.1 .11991 standard. fc145 dose in middle of inside $1 / 4^{\mathrm{s1}} \mathrm{n}$ shield

$c$ Total Dist from outside $=(204.7875+205.4225) / 2=205.1050 \mathrm{~cm}$ f145: $\quad 0205.1050200 .0$ o

fm145 2.0 \$ Factor of 2 times the ANS 6.1 .11991 standard. fc155 dose at 6 in. behind inside $n$ shield

c Total oist from outside $=205.4225+6 * 2.54=220.6225 \mathrm{~cm}$

155:n $0220.6625200 .0 \quad 0$

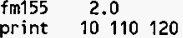

ctme 90.0

nps $\quad 1.005$ 
CRCH02P. INP $3.6 \mathrm{~kg}$ Pu Receipt Can with Hands, gamma ray calculation

PHOTON Dose Rate Calcs for calciner

c Hi 240Pu PuO2 Receipt Can Neutron Source is in Cell 1

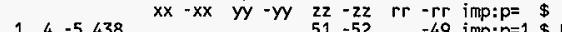

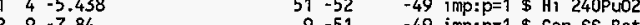

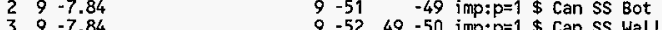

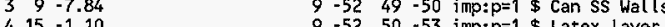

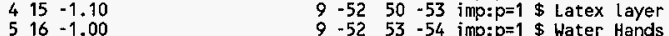

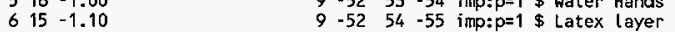

$710-1.29 \mathrm{e}-3 \quad 24-25 \quad 34-359-15 \quad(52: 55)$ imp: $\mathrm{p}=1$ \$ Inside Glovebox

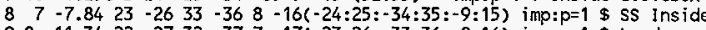

shield

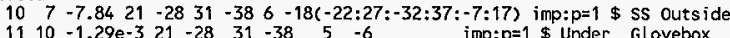

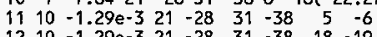

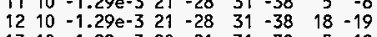

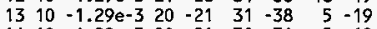

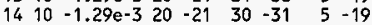

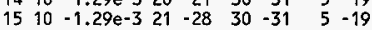

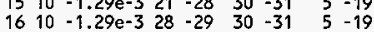

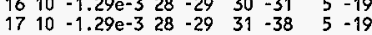

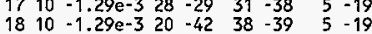

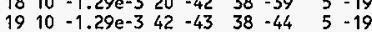

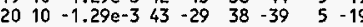

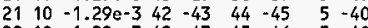

22 10 $-1.29 e-3 \quad 42 \quad-43 \quad 45 \quad-46 \quad 5 \quad-40$

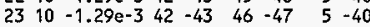

$24 \quad 10-1.29 e-3 \quad 42 \quad-43 \quad 47 \quad-48 \quad 5 \quad-40$

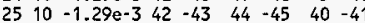

$26 \quad 10-1.29 e-3 \quad 42 \quad-43 \quad 45-46 \quad 40-41$

$\begin{array}{lllllllll}27 & 10 & -1.29 \mathrm{e}-3 & 42 & -43 & 46 & -47 & 40 & -41\end{array}$

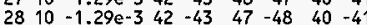

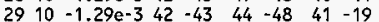

$\begin{array}{llllllllllll}30 & 10 & -1 & 290 & -3 & 42 & -43 & 48 & -38 & 41 & -19\end{array}$

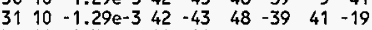

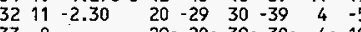

330 29:-20: 39:-30: $-4: 19$

imp: $p=1$ \$ Over Glovebox

imp:p=1 \$ Left Glovebox

imp: $p=1$ \$ Left Glovebox

imp: $p=1$ \$ Behind Glovebox

imp: $p=1$ \$ $R-B$ Glovebox

imp: $p=1$ \$ Right Glovebox

imp: $p=1$ \$ Front Glovebox

imp: $p=1$ \$ Front Glovebox

imp: $p=1$ \$ Front Glovebox

imp: $p=1$ \& Bot Outer 3/4"

imp: $p=1$ \$ Bot Middle 1 .

imp: $p=1$ \$ Bot Inner 3/4"

imp: $\mathrm{p}=1$ \& Bot Inside 1/4"

imp: $p=1$ \& Top Outer 3/41

imp: $p=1$ \& Top Middle 1 is

imp: $p=1$ Top Inner $3 / 4$ "

imp: $p=1$ Top Inside $1 / 4^{\prime \prime}$

imp: $p=1$ Top Inside $1 / 4 "$

imp:p=1 A Above shield

imp: $p=1$ \$ Behind $n$ shield

imp: $p=1$ \$ Behind $n$ shield

imp: $p=1 \$$ Concrete Floor

$\begin{array}{lr}c z & 7.7610 \\ c z & 8.4534 \\ c z & 14.2875 \\ \mathrm{pz} & -60.9600 \\ \mathrm{pz} & 0.0000 \\ \mathrm{pz} & 86.3600 \\ \mathrm{pz} & 86.5981 \\ \mathrm{pz} & 87.0744 \\ \mathrm{pz} & 87.3125 \\ \mathrm{pz} & 171.4500 \\ \mathrm{pz} & 171.7200 \\ \mathrm{pz} & 224.5663 \\ \mathrm{pz} & 233.6800 \\ \mathrm{pz} & 234.9500\end{array}$

$\$$ FEED TANK O.R. - (5/16"-0.040"1)

$\$$ FEED TANK O.R.

$\$$ FEED TANK FLANGE O.R.

$\$$ CONCRETE FLOOR bottom (24" thick)

$\$$ CONCRETE FLOOR surface

TLOVEBOX bottom surface

$\$$ GLOVEBOX Stainless Steel (3/32")

\$ GLOVEBOX Stainless Steel (3/32

\$ GLOVEBOX Lead Shield ( $\left.3 / 16^{\prime \prime}\right)$
$\$$ GLOVEBOX Stainless steel (3/32")

\$ GLOVEBOX Stainless Steel ( $3 / 32^{2}$ )

$\$$ FEED TANK Bottom $\mathrm{Flange}$ bottom

$\$$ FEED TANK Bottom Flange top

$\$$ FEED TANK TOP Flange bottom

$\$$ FEED TANK TOP Flange top

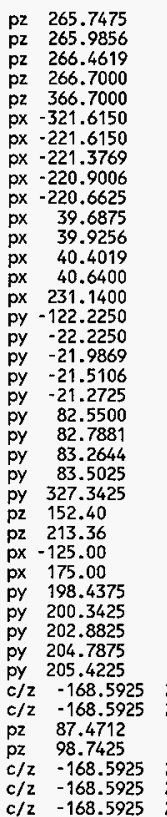

$\$$ GLOVEBOX top inside surface

$\$$ GLOVEBOX Stainless steel (3/32")

$\$$ GLOVEBOX Lead Shield (3/16")

\$ GLOVEBOX Stainless steel (3/32")

$\$$ MODEL TOP (GB roof $+100 \mathrm{~cm}$ )

$\$$ MODEL LEFT SIDE (GB side $+100 \mathrm{~cm}$ )

$\$$ GLOVEBOX left surface

GLOVEBOX Stainless steel (3/32'12)

GLOBOX stainless steel (3/32

$\$$ GLOVEBOX Stainless Steel (3/32")

\$ GLOVEBOX right inside surface

\$ GLOVEBOX Stainless Steel (3/32")

$\$$ GLOVEBOX Lead Shield (3/16")

\$ GLOVEBOX Stainless Steel (3/32")

$\$$ MODEL RIGHT SIDE (GB side +75")

$\$$ MODEL BACK SIDE (GB back $+100 \mathrm{~cm}$ )

$\$$ GLOVEBOX back surface

\$ GLOVEBOX Stainless steel (3/32")

$\$$ GLOVEBOX Lead Shield (3/16")

\$ GLOVEBOX Stainless Steel (3/32")

$\$$ GLOVEBOX front inside surface

$\$$ GLOVEBOX Stainless steel (3/32")

$\$$ GLOVEBOX Stainless \$teel $(3 / 32$

\$ GLOVEBOX Lead Shield (3/16")

\$ GLOVEBOX Stainless Steel (3/32")
$\$$ MODEL FRONT SIDE (GB front +96")

$\$ 51$ High $n$ shield

$\$+2$ ' Top $n$ shield

$\$$ Left End $n$ Shield

$\$$ Right End $n$ Shield

$\$ 3 / 4 "$ n Shield Material

$\$ 1.0$ " n Shield Material

3/4" n shield Material

1/4" n shield Material

Th" nhield Material

$29.8450 \quad 4.6038$ \&eceipt Can Inside Radius

$29.8450 \quad 4.7625$ Receipt Can Outside Radius $\$$ Receipt Can Stainless Steel Floor

$\$$ Recejpt Can Height

29.84507 .5406 \$ Water Hand Outside Radius

mode $w g t \stackrel{P}{=} 3600 * 3 \cdot 169 \mathrm{E}+8=1.141 \mathrm{E}+12$ Low $240 \mathrm{Pu}$

c $w g t=3600 * 1.115 \mathrm{E}+9=4.014 \mathrm{E}+12 \mathrm{Mid} 240 \mathrm{Pu}$

wgt $=3600 * 1.682 \mathrm{E}+9=6.055 \mathrm{E}+12$ High $240 \mathrm{Pu}$

sdef cel $=1$ erg=d5 pos $=-168.592529 .84500$

rad= d3 ext=d4 axs $=00 ;$ wgt $=6.055 \mathrm{E}+12$

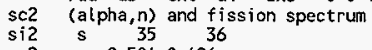

$\mathrm{sp2}$

$\operatorname{sc} 3$

$\begin{array}{ll}\mathrm{si3} & 4.6038 \\ \mathrm{sp} & -211\end{array}$ 


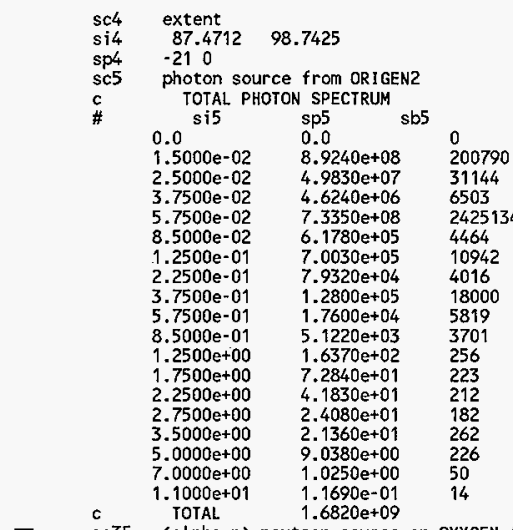

sc35 (alpha, $n$ ) neutron source on OXYGEN from Jacom and Liskien

c Energy of the alpha particles $=5.5 \mathrm{MeV}$

$\begin{array}{ll}2.5 & 2.43 \\ 2.6 & 2.34 \\ 2.7 & 2.73 \\ 2.8 & 2.06 \\ 2.9 & 2.00 \\ 3.0 & 1.88 \\ 3.1 & 1.66 \\ 3.2 & 1.56 \\ 3.3 & 1.34 \\ 3.4 & 1.09 \\ 3.5 & 0.92 \\ 3.6 & 0.79 \\ 3.7 & 0.64 \\ 3.8 & 0.55 \\ 3.9 & 0.48 \\ 4.0 & 0.39 \\ 4.1 & 0.35 \\ 4.2 & 0.23 \\ 4.3 & 0.18 \\ 4.4 & 0.11 \\ 4.5 & 0.07\end{array}$

spontaneous fission source spectrum (Pu240)

sp36 -3 $0.799 \quad 4.903$.

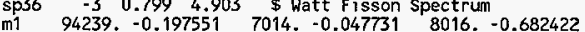

1001. $-0.065371 \$ P U(N 03) 4711.69 \mathrm{~g} / \mathrm{L}, \mathrm{H} 201000 \mathrm{~g} / \mathrm{L}$.

$\mathrm{m} 294238 .-0.00008994239 .-0.82309094240 .-0.054516$

94241. $-0.00231694242 .-0.00035695241 .-0.001603$

8016. $-0.118030 \$$ PUO2 for LOW 240 weight \% [CALCINE2.WQ1]

$\mathrm{m3} \quad 94238 .-0.00131294239 .-0.748841$ 94240. -0.108876

8016. - 0.117971 P P02 for Med 240 Weight \% [CALCINE2.WQ1]

$m 494238$. -0.001559 94239. -0.69358194240 . -0.154553

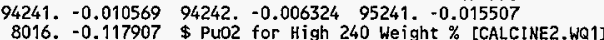

8016. -0.117907

$\$$ PuO2 for High 240 Weight \% [CALC
$5010 .-0.007986$ 5011. -0.032386

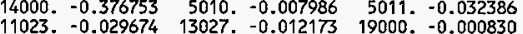

8016 . $-0.540198 \$ 7740$ PYREX TYPe I Glass [CALCINE1.WQ1]

26000. -0.74 24000. $-0.18 \quad 28000$. -0.08 \$ SS 304L Flanges

26000. -0.98 28000. $-0.02 \$$ Stainless Steel Glovebox

82000 . -1.00

26000 .

7014. -.78084 8016. $\quad-0.20946 \$ 18000$.

Lead Glovebox Shield

m10 7014. $=.78084$ 8016. -0.20946 18000. -0.00934

1001. $-.00036 \$ \mathrm{Ajr}$

13027. $-.034 \quad 20000 \cdot-0.044 \quad 26000 .-0.014$

11023. - .029 \$ Concrete (common Portland) ARH-600 11.F.1-3

m12

1001.0 .0854 5010. -0.0590 5011. -0.2410 \$ Neutron

$26000-0.0038$ 13027. -0.0004 25055. $-0.0002 \$ 210$

m13 8016. $-0.36496000 .-0.3439$ 13027. $-0.1906 \$$ Neutron

1001. -0.078420000 . -0.0123 14000. -0.0009 \& Shield

$5010,-0.00138$ 5011. -0.00562 12000. $-0.0006 \$ 207$

11023. -0.0006 39089. -0.0005 26000. -0.0001

$19000,-0.000116032,-0.0001 \$$ Y89 substituted for $\mathrm{sr}$. 


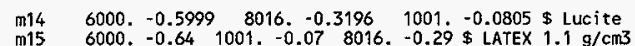
m16 1001. 0.333333 8016. 0.666667 . -0.29 \& LATEX $1.1 \mathrm{~g} / \mathrm{cm3}$ Photon dose conversion factors from ANS 6.1 .11991

$\begin{array}{ccccccc}c & \text { Photon dose conversion } \\ \text { den } & \log 0.010 & 0.015 & 0.02 & 0.03 & 0.04 & 0.05 \\ & 0.06 & 0.08 & 0.10 & 0.15 & 0.2 & 0.3 \\ & 0.4 & 0.5 & 0.6 & 0.8 & 1.0 & 1.5 \\ & 2.0 & 3.0 & 4.0 & 5.0 & 6.0 & 8.0\end{array}$

df0 $\quad \log 2.232 \mathrm{e}-05 \quad 5.652 \mathrm{e}-05 \quad 8.568 \mathrm{e}-05 \quad 1.184 \mathrm{e}-04 \quad 1.314 \mathrm{e}-04 \quad 1.382 \mathrm{e}-04$

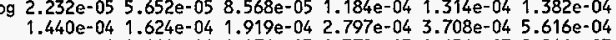

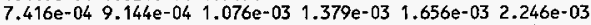
$2.758 \mathrm{e}-03 \quad 3.672 \mathrm{e}-03 \quad 4.500 \mathrm{e}-03 \quad 5.292 \mathrm{e}-03 \quad 6.012 \mathrm{e}-03 \quad 7.488 \mathrm{e}-03$ $8.892 \mathrm{e}-03 \quad 1.040 \mathrm{e}-02$

c Photon dose conversion factors from ANS 6.1.1 1977

$\begin{array}{rrrrrr}\log 0.01 & 0.03 & 0.05 & 0.07 & 0.10 & 0.15 \\ 0.20 & 0.25 & 0.30 & 0.35 & 0.40 & 0.45 \\ 0.50 & 0.55 & 0.60 & 0.65 & 0.70 & 0.80 \\ 1.00 & 1.40 & 1.80 & 2.20 & 2.60 & 2.80 \\ 3.25 & 3.75 & 4.25 & 4.75 & 5.00 & 5.25 \\ 5.75 & 6.25 & 6.75 & 7.50 & 9.00 & 11.00\end{array}$

df4 $\log 3.960 \mathrm{e}-03 \quad 5.820 \mathrm{e}-04 \quad 2.900 \mathrm{e}-04 \quad 2.580 \mathrm{e}-04 \quad 2.830 \mathrm{e}-04 \quad 3.790 \mathrm{e}-04$

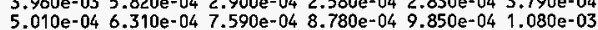

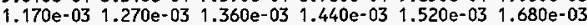

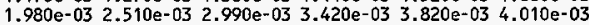
$4.410 \mathrm{e}-03 \quad 4.830 \mathrm{e}-03 \quad 5.230 \mathrm{e}-03 \quad 5.600 \mathrm{e}-03 \quad 5.800 \mathrm{e}-03 \quad 6.010 \mathrm{e}-03$ $6.370 \mathrm{e}^{-03} 6.740 \mathrm{e}-03 \quad 7.110 \mathrm{e}-03 \quad 7.660 \mathrm{e}^{-03} 8.770 \mathrm{e}-03 \quad 1.030 \mathrm{e}-02$ $1.180 \mathrm{e}-02,1.330 \mathrm{e}-02$

in hands modeled by water 1 inch thick (Cell. 5)

$\begin{array}{lc}f 4: \mathrm{p} & \mathrm{dos}\end{array}$

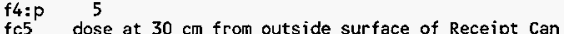

fc5 dose at $30 \mathrm{~cm}$ from outside surface of Receipt

f5:p $\quad-168.592564 .448893 .106850$

fc15 dose at $30 \mathrm{~cm}$ from outside surface of Glovebox

c Dist $=30.0+83.5025=113.5025 \mathrm{~cm}$

fc25 dose at 5 feet from outside surface of Glovebox

c $\quad Y$ Dist $=152.8+83.5025=235.9025 \mathrm{~cm}$

f25:p $\quad-168.5925 \quad 235.9025 \quad 93.10685 \quad 0$

fc35 dose at 6 in. in front of lower $n$ shield

c Total Dist from outside $=198.4375-6 * 2.54=183.1975 \mathrm{~cm}$

f35:p 0183.1975135 .0 0

fc45 dose in middle of outer $3 / 4^{1:}$ PYREX $n$ shield

Total Dist from outside $=(198.4375+200.3425) / 2=199.3900 \mathrm{~cm}$

f45: $0199.3900135 .0 \quad 0$

fc55 dose in middle of $1.011 \mathrm{n}$ shield

c Total Dist from outside $=(200.3425+202.8825) / 2=201.6125 \mathrm{~cm}$ f55:p 0201.6125 135.0

fc65 dose in middle of inner 3/4" PYREX $n$ shield

c Total Dist from outside $=(202.8825+204.7875) / 2=203.8350 \mathrm{~cm}$ f65:p $\quad 0203.8350135 .0 \quad 0$

fc75 dose in middle of inside $1 / 4 " n$ shield

c Total Dist from outside $=(204.7875+205.4225) / 2=205.1050 \mathrm{~cm}$ $f 75: p \quad 0205.1050135 .0 \quad 0$

fc85 dose at 6 in. behind inside $n$ shield

$c \quad$ Total Dist from outside $=205.4225+6 \star 2.54=220.6225 \mathrm{~cm}$

$85: p \quad 0220.6625135 .0 \quad 0$

f95 dose at 6 in. in front of upper $n$ shield

Total Dist from outsid

co5:p Total Dist from outside $=198.4375-6 * 2.54=183.1975 \mathrm{~cm}$

c Total Dist from outside $=(198.4375+200.3425) / 2=199.3900 \mathrm{~cm}$

105:p 0199.3900200 .0

fc115 dose in middle of $1.01 \mathrm{n}$ shield

$c$ Total Dist from outside $=(200.3425+202.8825) / 2=201.6125 \mathrm{~cm}$ f115:p $0201.6125200 .0 \quad 0$

fc125 dose in middle of inner 3/4" PYREX $n$ Shield

c Total Dist from outside $=(202.8825+204.7875) / 2=203.8350 \mathrm{~cm}$ f125:p $0203.8350200 .0 \quad 0$

fc135 dose in middle of inside 1/4" $n$ shield

c Total Dist from outside $=(204.7875+205.4225) / 2=205.1050 \mathrm{~cm}$ f135: $\mathrm{p} \quad 0205.1050200 .0$

fc145 dose at 6 in. behind inside $n$ shield

c Total Dist from outside $=205.4225+6 * 2.54=220.6225 \mathrm{~cm}$

f145:p 0220.6625200 .0

print 10110120

ctme 90.0

nps $3 . e 6$ 
HNF-SD-CP-TI-218 Rev. 0

APPENDIX E

MISCELLANEOUS REFERENCE PAGES

Calciner Shield wall Specifications

7740 Corning Trademark PYREX ${ }^{\$ 5}$ Composition for Feed Tank Glass

Physical Properties Glass Composition of Code 7740 Glass

Physical Dimensions of Glass Pipe Straight Lengths

Plutonium Nitrate Feed material composition by Jerome F. Durni1

Material Data for Borated Polyethylene products 210, 207 by Scott Huneycutt

${ }^{5}$ PYREX is a registered trademark of the Corning Company. 
HNF-SD-CP-TI-218 Rev. 0

This page intentionally left blank 


\section{Calciner Shield Wall Specifications.}

\section{Scope}

A shield wall will need to be constructed to minimize the dose from the calciner components to the worker located at the calciner control panel. The upper portion of this wall will need to be transparent for security purposes.

\section{Design Criteria}

The optimum design for a shield to reduce exposure from neutron radiation should consist of three layers. The first layer, the layer closest to the neutron source, should be a hydrogenous material to thermalize the neutron energy spectra. The second layer should be a Borated material to capture the thermal neutrons. The third layer, the layer most distant from the neutron source, should be a high $Z$ material, such as steel or lead, to attenuate the gamma rays produced from the capture of the neutrons in the Borated material.

Note:

The suggested shield design is one possible configuration based on preliminary dose-rate calculations. Further analysis is being conducted.

Due to security and fire safety requirements, this shield wall will have a slightly different layer configuration.

Lower Portion (approximately 5 feet)

The first layer of the lower portion of the shield wall, the layer closest to the calciner glovebox, should have a $19.0 \mathrm{~mm}\left(3 / 4^{3}\right)$ layer of 7740 PYREX ${ }^{\otimes}$ glass. The second layer should have a $25.4 \mathrm{~mm}$ ( 1 ") layer of Borated Polyethylene \#210 which has a $30 \%$ Boron content, The third layer should be a $6.4 \mathrm{~mm}$ (1/4") layer of Stainless Steel. Upper Portion (approximately 2 feet )

The first layer of the upper portion of the shield wall, the layer closest to the calciner glovebox, should have a $19.0 \mathrm{~mm}$ ( $3 / 4$ ") layer of 7740 PYREX 0 glass. The second layer should have a $25.4 \mathrm{~mm}$ ( I") layer of clear Acrylic, Lexan, or Plexiglas. The third layer should be another layer of $19.0 \mathrm{~mm}\left(3 / 4^{\prime \prime}\right)$ layer of 7740 PYREX $\odot$ glass. A fourth layer will be required. The fourth layer should be a $6.4 \mathrm{~mm}$ (1/4") layer of $2.0 \mathrm{~mm}$ equivalent Leaded Glass.

These sandwiched materials will need to be. fit together in a way that no two seams lineup to produce a gap in the shield. 


\section{Materials}

Listed below are some possible Vendors, their contacts and latest cost estimate, for the required materials.

Vendor

PYREX $\odot 740$

ABRISA Industrial Glass, Inc.

John Kolinke

1456 Fleet Ave.

PO Box 3053

Ventura, CA 93003

(800) $350-5000$

Borated Polyethylene

Catalog \# 210

Reactor Experiments, Inc.

Dean White, ext. 352

1275 Hammerwood Ave.

Sunnyvale, CA 94088-2231

(408) $745-6770$

Leaded Glass $(=2.0 \mathrm{~mm} \mathrm{~Pb})$

Shielding International

Bill Chisolm

182 NW Earl Street

PO Box Z

Madras, OR $97741-0069$

(800) 292-2247

Leaded Glass

Hot Cell Services

$2262685^{\text {th }}$ Place South

Kent, WA 98031

Mike Caulkworth

(206) $854-4945$
Thickness. Sheet size Est. Price

$19.0 \mathrm{~mm} \quad 305 \mathrm{~mm} \times 610 \mathrm{~mm} \quad 255.00$

(0.75") (12"' $) 24 ")$

$845 \mathrm{~mm} \times 1150 \mathrm{~mm} \quad 768.00$

- $\left(33.25^{\prime \prime} \times 45.25^{\prime \prime}\right)$

[sheet edges are unfinished]

[comes in various thicknesses (3/32" to $21 / 4$ ")]

$25.0 \mathrm{~mm} \quad 610 \mathrm{~mm} \times 610 \mathrm{~mm}$

(1.0") (24" $\left.\times 24^{\prime \prime}\right)$

$1219 \mathrm{~mm} \times 1219 \mathrm{~mm}$

(48" $\left.\times 48^{\prime \prime}\right)$

$6.4 \mathrm{~mm} \quad \hat{2} 05 \mathrm{~mm} \times 305 \mathrm{~mm}$

$(0.25 ")$. (12"x 12")

to

$914 \mathrm{~mm} \times 1220 \mathrm{~mm}$

$\left(36^{\prime \prime} \times 48^{\prime \prime}\right)$

$6.4 \mathrm{~mm}$

$\left(0.25^{\prime \prime}\right)$ 
HNF-SD-CP-TI-218 ReV. 0

7740 Corning Trademark PYREX Composition for Feed Tank Glass

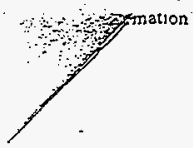

http:/huw.cominglabware.com/techpages/techpagenf $6 . \mathrm{html}$

Warnings
1. Do not use in heat applications.

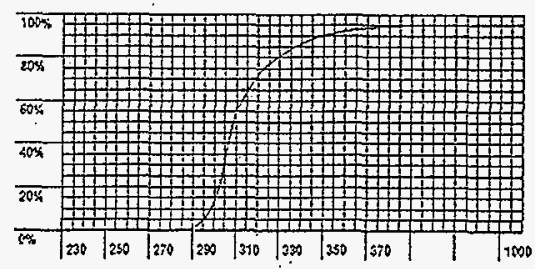

7740
Corning Trademark: $\quad \begin{aligned} & \text { Common Names } \\ & \text { BYREX } \$\end{aligned}$

PYREX®

\section{Standards}

Type I, Class A Borosilicate conforming to federal specification DD-G-54 Ib and ASTM E-438. Also meets the U.S. Pharmacopocia specs for Type I Borosilicate Glass.

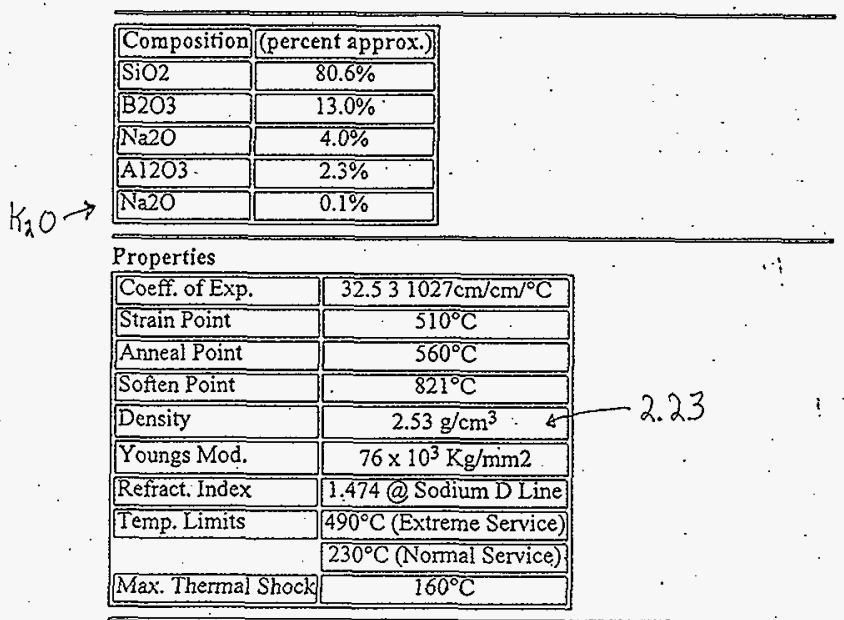

\section{Applications}

Designed for use in all products requiring very high resistance to strong acids, alkalis and products intended for use in heat applications such as 
HNF-SD-CP-TI-218 Rev. 0

Physical Properties Glass Composition of Code 7740 Glass

\section{BASIC ENGINEERING DATA}

\section{CHEMICAL PROPERTIES}

Chemical Durability

All of Corning's piping systems are resistant to more acids and acidic materials than any other pipe.

By exception there are only these chemicals to avoid: hydrofiuoric acid and hot alkelies. See the two graphs for more specific data. The graphs apply only to Corning's Code 7740 giass and show orderof-magnitude data, since operating conditions greetly affect periormance.

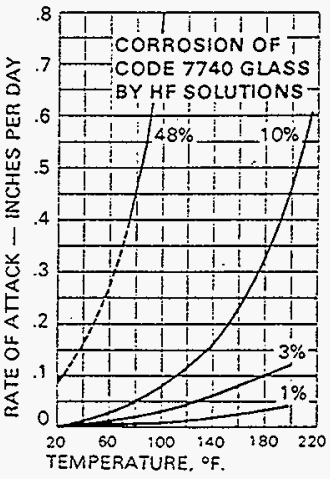

Concentrated hrdrofluoric acio attacks Code 7740 glass at all temperatures. Mild solutions dumped into drainines can be tolerated at room temperetures.

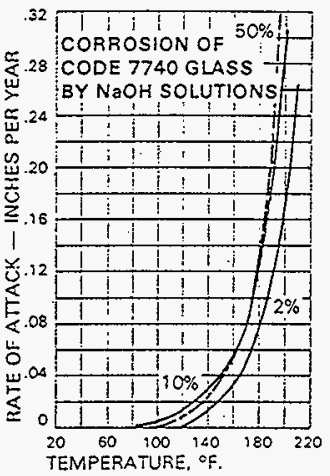

Alkaline solutions arack glass very slowly at room temperatures, but as temperature is increased orer $700^{\circ} \mathrm{F}$, the corrosion rate ises rapidly.

\section{PHYSICAL PROPERTIES}

Glass Composition

Ptrysical Properties Of Code 7740 Glass

$$
4.0407 \%
$$

Mwke ?e

b... 9.39

$A / 0.98$

$K \quad 0.28$

No 3.25

Si

33.73

$\frac{i^{2} 36}{5-01}$
Composition

Coming's code 740 lowexpansion borosilicate glass has the following approximate chemical composition:

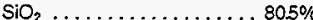

$\mathrm{Na}_{2} \mathrm{O} \ldots \ldots \ldots \ldots \ldots \ldots .4 .0 \%$

$\mathrm{K}_{2} \mathrm{O} \ldots \ldots \ldots \ldots \ldots \ldots \ldots \ldots$

$\mathrm{B}_{2} \mathrm{O}_{3} \ldots \ldots \ldots \ldots \ldots \ldots \ldots \ldots$

$\mathrm{Al}_{2} \mathrm{O}_{3} \ldots \ldots \ldots \ldots \ldots \ldots .2 .0 \%$

For further details please refer to ASTMC 599, "Standard Specification for Process Glass Pipe and Fittings."

Linear Coefficient of Expansion: (between $32^{\circ} \mathrm{F}$ and $572^{\circ} \mathrm{F} 10^{\circ} \mathrm{C}$ and $300^{\circ} \mathrm{C}$, per ASTM Method E 228)

$18.1 \times 10^{-3} \mathrm{in} / \mathrm{in} /{ }^{\circ} \mathrm{F}$

$32.5 \times 10^{-7} \mathrm{~cm} / \mathrm{cm} / 9 \mathrm{C}$

Annealing

All fittings and all straight lengths under $60^{\prime \prime}$ in length are annealed to reduce internal stress. This also makes the pipe under $60^{\prime \prime}$ easier to field tabricate.

\section{Tempering}

All pipe ends on lengths $60^{\prime \prime}$ and lonoer are tempered, the distance varying between $4^{\prime \prime}$ and $8^{\prime \prime}$ depending on the pipe system. The tempered area blends gradually into the center portion of the straight length. This is to permit pipe lengths to be cut in the central portion.

Thermal Conductivity: $0.73 \mathrm{Btw} / \mathrm{hr}-\mathrm{ft}^{2}{ }^{\circ} \mathrm{\circ} / \mathrm{F} / \mathrm{t}$

- $0.0035 \mathrm{cal} / \mathrm{sec}-\mathrm{cm}^{2} .0 \mathrm{C} / \mathrm{cm}$

Specific Heat:

$0.20 \mathrm{Btu} / \mathrm{b}-{ }^{\circ} \mathrm{F}$

$0.20 \mathrm{cal} / \mathrm{gm} \cdot{ }^{\circ} \mathrm{C}$

Dielectric Constant:

at $23^{\circ} \mathrm{C}$ and $1 \mathrm{M} \mathrm{Hz}$, per ASTM Method D 150: $4.6 \div 0.2$.

Density: Approximately $139 \mathrm{~b}^{2} / \mathrm{t}^{3}\left(2.23 \mathrm{gm} / \mathrm{cm}^{3}\right)$.

Young's Modulus: per ASTM Method C215: in the range of. $9 \times 10^{6}$ to $10 \times 10^{6} \mathrm{psi}^{\text {. }}$ 
HNF-SD-CP-TI-218 Rev. 0

Physical Dimensions of Glass Pipe Straight Lengths

CONICALSYSTEM PYREX ${ }^{7 m}$

PIPE

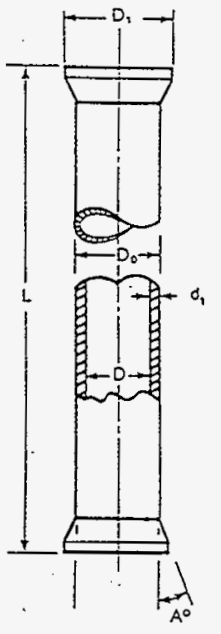

Straight Length

\begin{tabular}{|c|c|c|c|c|c|}
\hline $\begin{array}{l}\text { Pipe } \\
\text { Size } \\
\text { D }\end{array}$ & $\begin{array}{c}\text { Pipe O.D. } \\
\mathrm{D}_{0}\end{array}$ & $\begin{array}{c}\text { Cone O.D. } \\
D_{i}\end{array}$ & $\begin{array}{c}\text { Wall } \\
\text { Thickness } \\
d_{1}\end{array}$ & $\begin{array}{c}\text { Cone } \\
\text { Angle } \\
\text { A }\end{array}$ & $\begin{array}{l}\text { Approx: } \\
\text { Weight } \\
\text { Per Foot }\end{array}$ \\
\hline $\begin{array}{c}1^{\prime \prime} \\
(25 \mathrm{~mm})\end{array}$ & $\begin{array}{c}15 / 16^{\prime \prime} \pm .016^{\prime \prime} \\
(33 \mathrm{~mm} \pm 0.4 \mathrm{~mm})\end{array}$ & $\begin{array}{c}19 / 16^{\prime \prime} \pm .016^{\prime \prime} \\
(40 \mathrm{~mm} \pm 0.4 \mathrm{~mm})\end{array}$ & $\begin{array}{c}5 / 32^{\prime \prime} \pm .016^{\prime \prime} \\
(4.0 \mathrm{~mm} \pm 0.4 \mathrm{~mm})\end{array}$ & $12^{\circ}$ & $\begin{array}{c}0.6 \mathrm{bb} \\
(0.27 \mathrm{~kg})\end{array}$ \\
\hline $\begin{array}{c}51 / 2^{n} \\
(38 \mathrm{~mm})\end{array}$ & $\begin{array}{c}127 / 32^{n} \pm .020^{n} \\
(47 \mathrm{~mm} \pm 0.5 \mathrm{~mm})\end{array}$ & $\begin{array}{c}21 / 8^{\prime \prime} \pm .016^{\prime \prime} \\
(54 \mathrm{~mm} \pm 0.4 \mathrm{~mm})\end{array}$ & $\begin{array}{c}11 / 64^{\prime \prime} \doteq .016^{\prime \prime} \\
(4.4 \mathrm{~mm} \pm 0.4 \mathrm{~mm})\end{array}$ & $i 2^{\circ}$ & $\begin{array}{c}1.0 \mathrm{~kb} \\
(0.45 \mathrm{~kg})\end{array}$ \\
\hline $\begin{array}{c}2^{n} \\
(51 \mathrm{~mm})\end{array}$ & $\begin{array}{c}211 / 32^{\prime \prime} \pm 040^{\prime \prime} \\
(60 \mathrm{~mm} \pm 7.0 \mathrm{~mm})\end{array}$ & $\begin{array}{c}25 / 6^{\prime \prime} \pm .020^{\circ} \\
(67 \mathrm{~mm} \pm 0.5 \mathrm{~mm})\end{array}$ & $\begin{array}{c}11 / 64^{\prime \prime} \pm .020^{\prime \prime} \\
(4.4 \mathrm{~mm} \pm 0.5 \mathrm{~mm})\end{array}$ & $12^{\circ}$ & $\begin{array}{r}1.13 \mathrm{~b} \\
(0.51 \mathrm{~kg}) \\
\end{array}$ \\
\hline $\begin{array}{c}3^{\prime \prime} \\
(76 \mathrm{~mm}) \\
\end{array}$ & $\begin{array}{c}3^{13} 33^{n} \pm .056^{\prime \prime} \\
(87 m m=1.4 \mathrm{~mm})\end{array}$ & $\begin{array}{c}325 / 32^{\prime \prime} \pm .031^{\prime \prime} \\
(96 \mathrm{~mm} \pm 0.8 \mathrm{~mm})\end{array}$ & $\begin{array}{c}13 / 66^{\prime \prime} \pm .021^{\prime \prime} \\
(5.2 \mathrm{~mm} \pm 0.5 \mathrm{~mm})\end{array}$ & $12^{\circ}$ & $\begin{array}{c}2.0 \mathrm{lb} \\
(0.91 \mathrm{~kg}) \\
\end{array}$ \\
\hline $\begin{array}{c}4^{\prime \prime} \\
(702 \mathrm{~mm})\end{array}$ & $\begin{array}{c}4^{17 / 32} " \pm .068^{\prime \prime} \\
(715 \mathrm{~mm} \pm .7 \mathrm{~mm})\end{array}$ & $\begin{array}{c}520 / 64^{\prime \prime} \pm .016^{n} \\
(136 \mathrm{~mm} \pm 0.4 \mathrm{~mm})\end{array}$ & $\begin{array}{c}17 / 6 \mathrm{e}^{n} \pm 025^{n} \\
(6.7 \mathrm{~mm} \pm 0.6 \mathrm{~nm})\end{array}$ & $21^{\circ}$ & $\begin{array}{c}3.4 \mathrm{lb} \\
(1.5 \mathrm{~kg})\end{array}$ \\
\hline $\begin{array}{c}6^{\prime \prime} \\
(152 \mathrm{~mm})\end{array}$ & $\begin{array}{c}6^{21 / 32} \div .075^{\prime \prime} \\
(169 \mathrm{~mm} \pm 7.9 \mathrm{~mm})\end{array}$ & $\begin{array}{c}7553^{\prime \prime} \pm .016^{\prime \prime} \\
(792 \mathrm{~mm} \pm 0.4 \mathrm{~mm})\end{array}$ & $\begin{array}{c}5 / 16^{\prime \prime} \pm .040^{\prime \prime} \\
(7.9 \mathrm{~mm} \pm 1.0 \mathrm{~mm})\end{array}$ & $21^{\circ}$ & $\begin{array}{c}63 \mathrm{lb} \\
(2.9 \mathrm{~kg})\end{array}$ \\
\hline
\end{tabular}

$\therefore$ j $19 i:$ in ir $\because ;=$

Codes

\begin{tabular}{|c|c|c|c|c|c|c|}
\hline $\begin{array}{c}\text { Pipe } \\
\text { Size }\end{array}$ & \multicolumn{1}{|c|}{ Minimum Length } & $\begin{array}{c}6^{\prime \prime} \mathrm{L} \\
(752 \mathrm{~mm})\end{array}$ & $\begin{array}{c}12^{\prime \prime} \mathrm{L} \\
(305 \mathrm{~mm})\end{array}$ & $\begin{array}{c}18^{\prime \prime} \mathrm{L} \\
(457 \mathrm{~mm})\end{array}$ & $\begin{array}{c}24^{\prime \prime} \mathrm{L} \\
(670 \mathrm{~mm})\end{array}$ \\
\hline $\begin{array}{c}1^{\prime \prime} \\
(25 \mathrm{~mm})\end{array}$ & $\begin{array}{c}3^{n} \\
(70 \mathrm{~mm})\end{array}$ & $72-1590$ & $72-1550$ & $72-7010$ & $72-7015$ & $72-7020$ \\
\hline $\begin{array}{c}11^{\prime \prime} \\
(38 \mathrm{~mm})\end{array}$ & $\begin{array}{c}312^{\prime \prime} \\
(89 \mathrm{~mm})\end{array}$ & $72-1600$ & $72-1560$ & $72-7100$ & $72-7105$ & $72-7110$ \\
\hline $\begin{array}{c}2^{n} \\
(51 \mathrm{~mm})\end{array}$ & $\begin{array}{c}4^{n} \\
(102 \mathrm{~mm})\end{array}$ & $72-1610$ & $72-1570$ & $72-7190$ & $72-7195$ & $72-7200$ \\
\hline $\begin{array}{c}3^{n} \\
(76 \mathrm{~mm})\end{array}$ & $\begin{array}{c}5^{n} \\
(127 \mathrm{~mm})\end{array}$ & $72-1620$ & $72-1580$ & $72-7280$ & $72-7285$ & $72-7290$ \\
\hline $\begin{array}{c}4^{n} \\
(102 \mathrm{~mm})\end{array}$ & $\begin{array}{c}6^{\prime \prime} \\
(152 \mathrm{~mm})\end{array}$ & $72-2560$ & $72-2560$ & $72-7570$ & $72-7375$ & $72-7380$ \\
\hline $\begin{array}{c}6^{\prime \prime} \\
(152 \mathrm{~mm})\end{array}$ & $\begin{array}{c}6^{\prime \prime} \\
(152 \mathrm{~mm})\end{array}$ & $72-2610$ & $72-2610$ & $72-1360$ & $72-1365$ & $72-1380$ \\
\hline
\end{tabular}

\section{Codes}

\begin{tabular}{|c|c|c|c|c|c|c|c|c|c|}
\hline $\begin{array}{l}\text { Pipe } \\
\text { Size }\end{array}$ & $\begin{array}{c}30^{n \mathrm{~L}} \mathrm{~L} \\
(762 \mathrm{~mm})\end{array}$ & $\begin{array}{c}36^{\prime \prime} \mathrm{L} \\
(914 \mathrm{~mm})\end{array}$ & $\begin{array}{c}48^{\prime \prime} \mathrm{L} \\
(1219 \mathrm{~mm})\end{array}$ & \begin{tabular}{|c}
$60^{\circ} \mathrm{L}$ \\
$(7524 \mathrm{~mm})$
\end{tabular} & $\begin{array}{c}72^{\prime \prime} \mathrm{L} \\
(1829 \mathrm{~mm})\end{array}$ & $\begin{array}{c}84^{n} \mathrm{~L} \\
(2134 \mathrm{~mm})\end{array}$ & $\left.\begin{array}{c}96^{\prime \prime} \mathrm{L} \\
(2438 \mathrm{~mm})\end{array}\right)$ & $\begin{array}{c}108^{n \mathrm{~L}} \\
(2473 \mathrm{~mm})\end{array}$ & $\begin{array}{r}120^{\circ} \mathrm{L} \\
(3048 \mathrm{~mm}\end{array}$ \\
\hline $\begin{array}{c}11 " \\
(25 \mathrm{~mm})\end{array}$ & $72-7540$ & 72.7030 & 72.7040 & 72.7050 & $72-7500$ & $72-7070$ & $72-7510$ & 72.7520 & $72-4500$ \\
\hline $\begin{array}{l}\left.111^{n}\right) \\
(38 \mathrm{~mm})\end{array}$ & 72.7530 & 72.7120 & $72-7130$ & 72.740 & $72-7501$ & $72-7160$ & $72-7511$ & $72-7521$ & $72-4510$ \\
\hline $\begin{array}{c}2^{\prime \prime \prime} \\
(5 \mathrm{~mm})\end{array}$ & 72.7541 & 72.7210 & 72.7220 & $72-7230$ & $72-7502$ & $72-7250$ & 72.7512 & 72.7522 & $72-4520$ \\
\hline $\begin{array}{c}3^{\prime \prime} \\
(6 \mathrm{~mm})\end{array}$ & $72-7542$ & 72.7300 & $72-7310$ & $72-7320$ & $72-7503$ & 72.7340 & 72.7513 & 72.7523 & 724530 \\
\hline $\begin{array}{c}4^{\prime \prime} \\
(702 \mathrm{~mm})\end{array}$ & 72.7543 & 72.7390 & $72-7400$ & 72.7410 & 72.7504 & $72-7430$ & 72.7514 & 72.7524 & $72-4540$ \\
\hline (1:52mm & $72-7544$ & $72-1410$ & $72-1430$ & $72-1450$ & 72.7505 & 72.1470 & $72: 7515$ & 72.7525 & $72-1490$ \\
\hline
\end{tabular}


Plutonium Nitrate Feed material composition by Jerome $F$. Durni 7 .

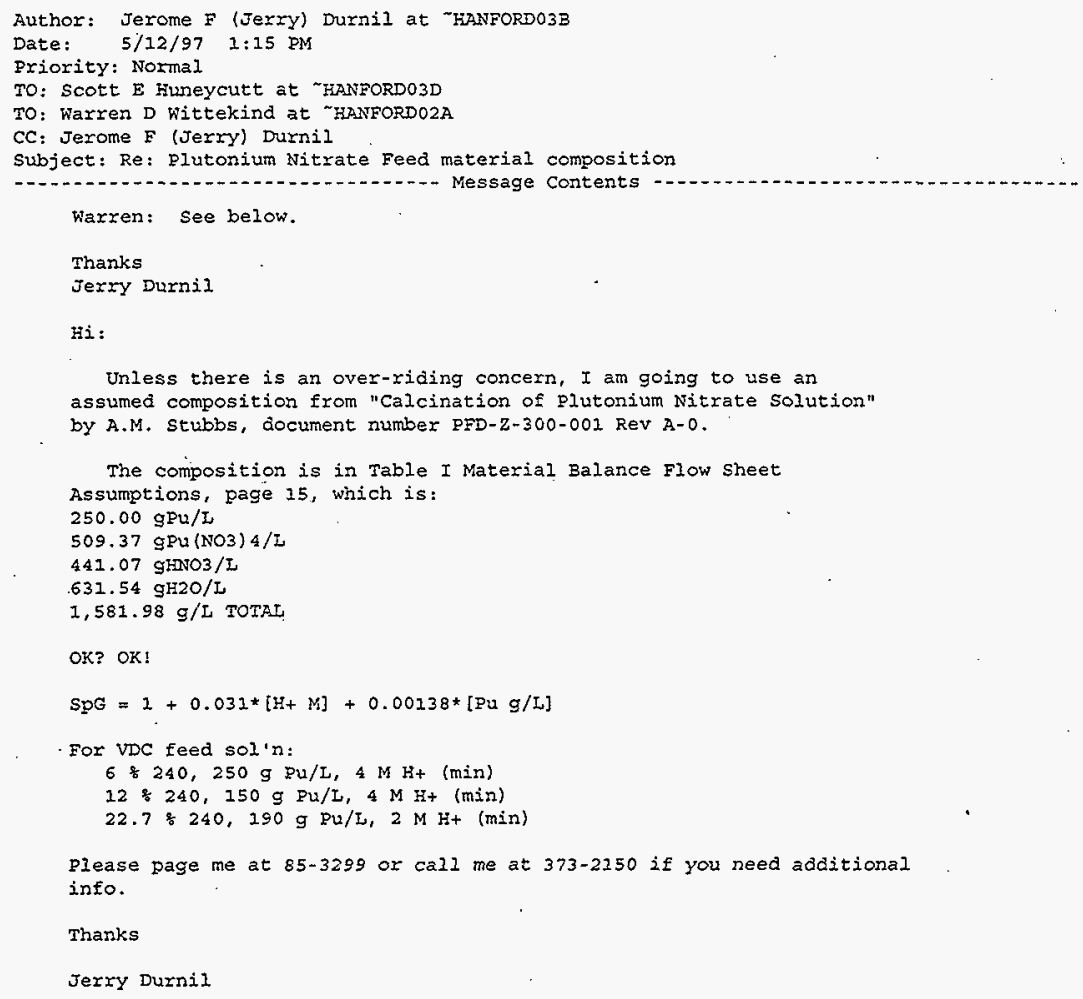




\section{HNF-SD-CP-TI-218 Rev. 0}

Material Data for Borated Polyethylene products 210, 207 by Scott Huneycutt

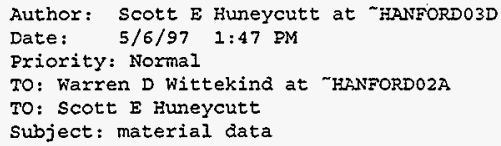

220

207

H
$\mathrm{B}$
$\mathrm{C}$
$\mathrm{AI}$
$\mathrm{Ca}$
$\mathrm{Fe}$
$\mathrm{K}$
$\mathrm{Mg}$
$\mathrm{Na}$
$\mathrm{O}$
$\mathrm{S}$
$\mathrm{Si}$
$\mathrm{Sr}$

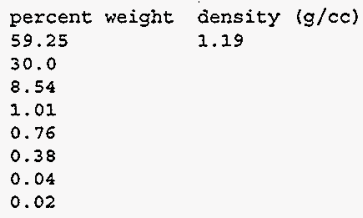

1.01

0.76

0.38

0.04

0.02

7.84

0.70

34.39

19.06

1.23

0.01

0.01

0.06

0.06

36.49

0.01

0.09

0.05

I hope these are of value.

scott 
HNF-SD-CP-TI-218 Rev. 0

This page intentionally left blank 


\section{DISTRIBUTION SHEET}

\begin{tabular}{|c|c|c|c|c|c|}
\hline \multirow{2}{*}{$\begin{array}{l}\text { To } \\
\text { Distribution }\end{array}$} & \multirow{2}{*}{\multicolumn{3}{|c|}{$\begin{array}{l}\text { From } \\
\text { Criticality and Shielding }\end{array}$}} & \multicolumn{2}{|l|}{ Page 1 of 1} \\
\hline & & & & \multicolumn{2}{|c|}{ Date $07 / 17 / 97$} \\
\hline \multirow{2}{*}{\multicolumn{4}{|c|}{$\begin{array}{l}\text { Project Title/Work Order } \\
\text { PFP Vertical Calciner Shield Wall Dose Rate Calculations Using } \\
\text { MCNP }\end{array}$}} & \multirow{2}{*}{\multicolumn{2}{|c|}{$\begin{array}{ll}\text { EDT No. } & 620293 \\
\text { ECN No. } & \text { N/A }\end{array}$}} \\
\hline & & & & & \\
\hline \multicolumn{2}{|l|}{ Name } & $\begin{array}{l}\text { Text } \\
\text { With All } \\
\text { Attach. }\end{array}$ & Text Only & $\begin{array}{l}\text { Attach./ } \\
\text { Appendix } \\
\text { Only }\end{array}$ & $\begin{array}{l}\text { EDT/ECN } \\
\text { Only }\end{array}$ \\
\hline $\begin{array}{l}\text { G.G. Bergquist } \\
\text { E.P. Bonadie } \\
\text { K.D. Dobbin } \\
\text { D.G. Erickson } \\
\text { S.R. Gedeon } \\
\text { M.W. Gibson } \\
\text { J.Greenborg } \\
\text { K.E. Hillesland } \\
\text { S.E. Huneycutt } \\
\text { S.F. Kessler } \\
\text { C.M. Kronval1 } \\
\text { J.S. Lan } \\
\text { W.S. Lewis } \\
\text { E.M. Miller } \\
\text { L.T. Nirider } \\
\text { S.E. Nunn } \\
\text { L.L. Pedersen (3) } \\
\text { A.L. Ramble } \\
\text { R.F. Richards } \\
\text { S.P. Roblyer } \\
\text { R.H. Ruben } \\
\text { K.N. Schwinkendorf } \\
\text { W.T. Watson } \\
\text { C.L. Whalen } \\
\text { W.D. Wittekind (2) }\end{array}$ & 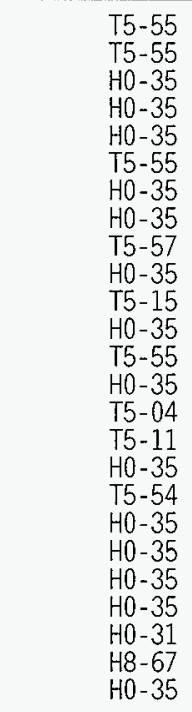 & $\begin{array}{l}x \\
x \\
x \\
x \\
x \\
x \\
x \\
x \\
x \\
x \\
x \\
x \\
x \\
x \\
x \\
x \\
x \\
x \\
x \\
x \\
x \\
x \\
x \\
x \\
x\end{array}$ & & & \\
\hline Central Files & $A 3-88$ & $x$ & & & \\
\hline
\end{tabular}

